\title{
Uncertainty in modelling the energy performance of buildings and the occupants
}

Feedback from practical experience of models

"You cannot be certain about uncertainty. "

ing. Ad van der $\mathrm{Aa}$

Cauberg-Huygen Consulting Engineers

The Netherlands

a.vanderaa@chri.nl 
II

\title{
Uncertainty in modelling the energy performance of buildings and the occupants
}

Feedback from practical experience of models

"You cannot be certain about uncertainty."

\author{
ing. Ad van der $\mathrm{Aa}$ \\ Cauberg-Huygen Consulting Engineers \\ The Netherlands \\ a.vanderaa@chri.nl
}



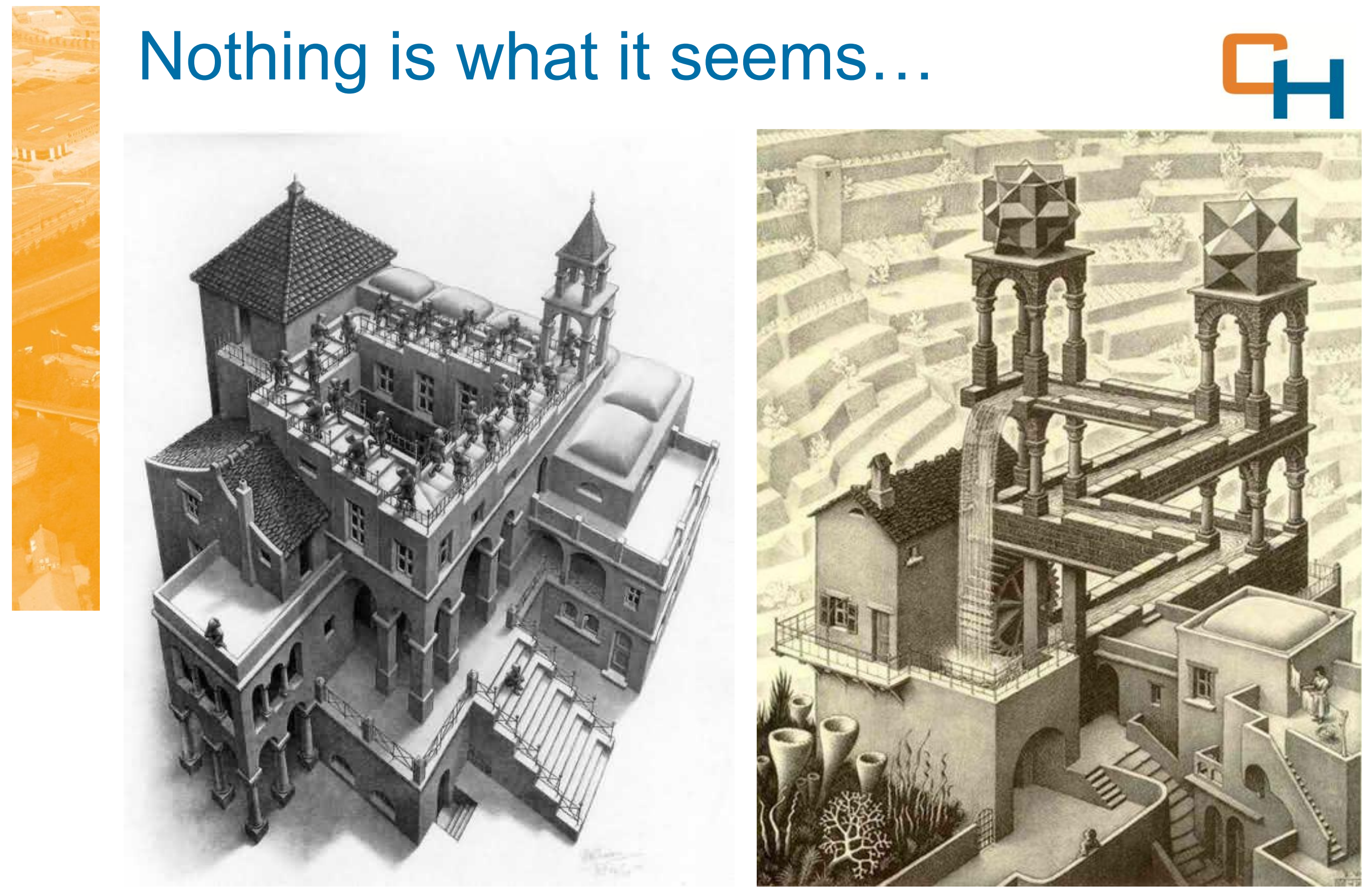


\section{Basic physics?}
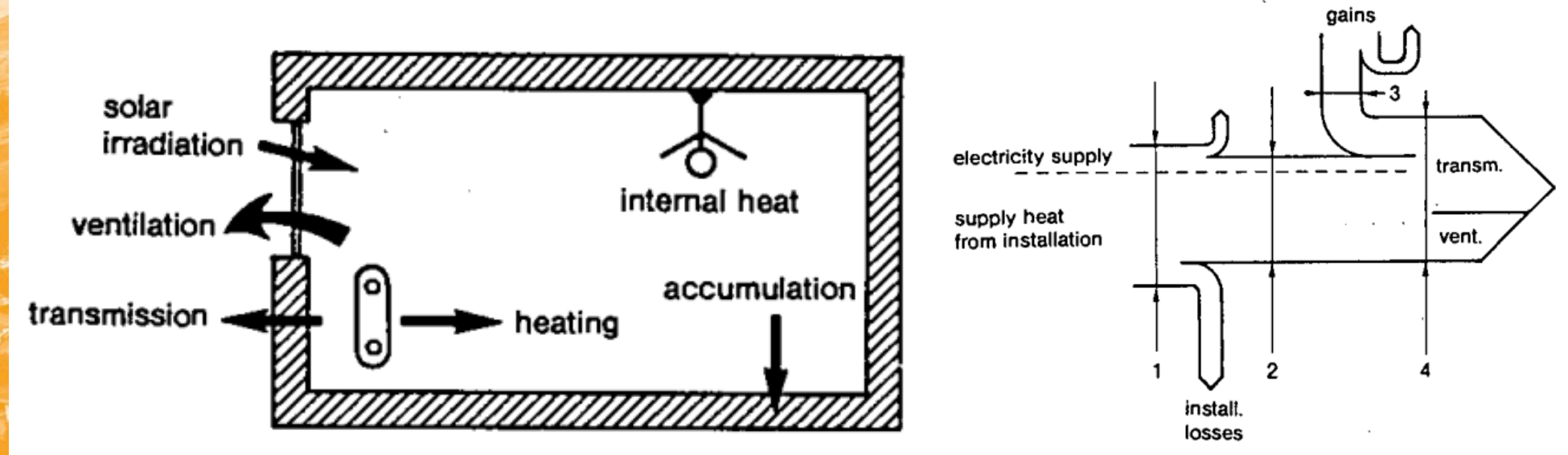

1 primary energy

2 net heat demand

3 useful gains

4 building heat loss

Figure 2: Apart from the efficiency of the heating installation, the heat balance of a building determines the energy consumption for space heating.

$\underline{\phi_{\mathrm{HD}}-\phi_{\mathrm{L}}+\phi_{\mathrm{ACC}}-\phi_{\mathrm{G}}}$

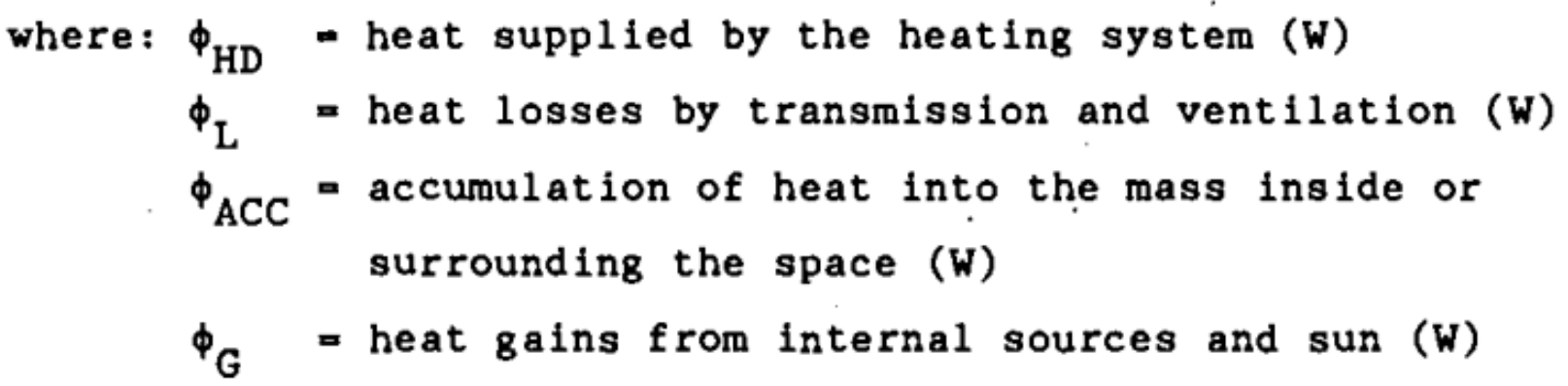




\section{Is it really that simple?}

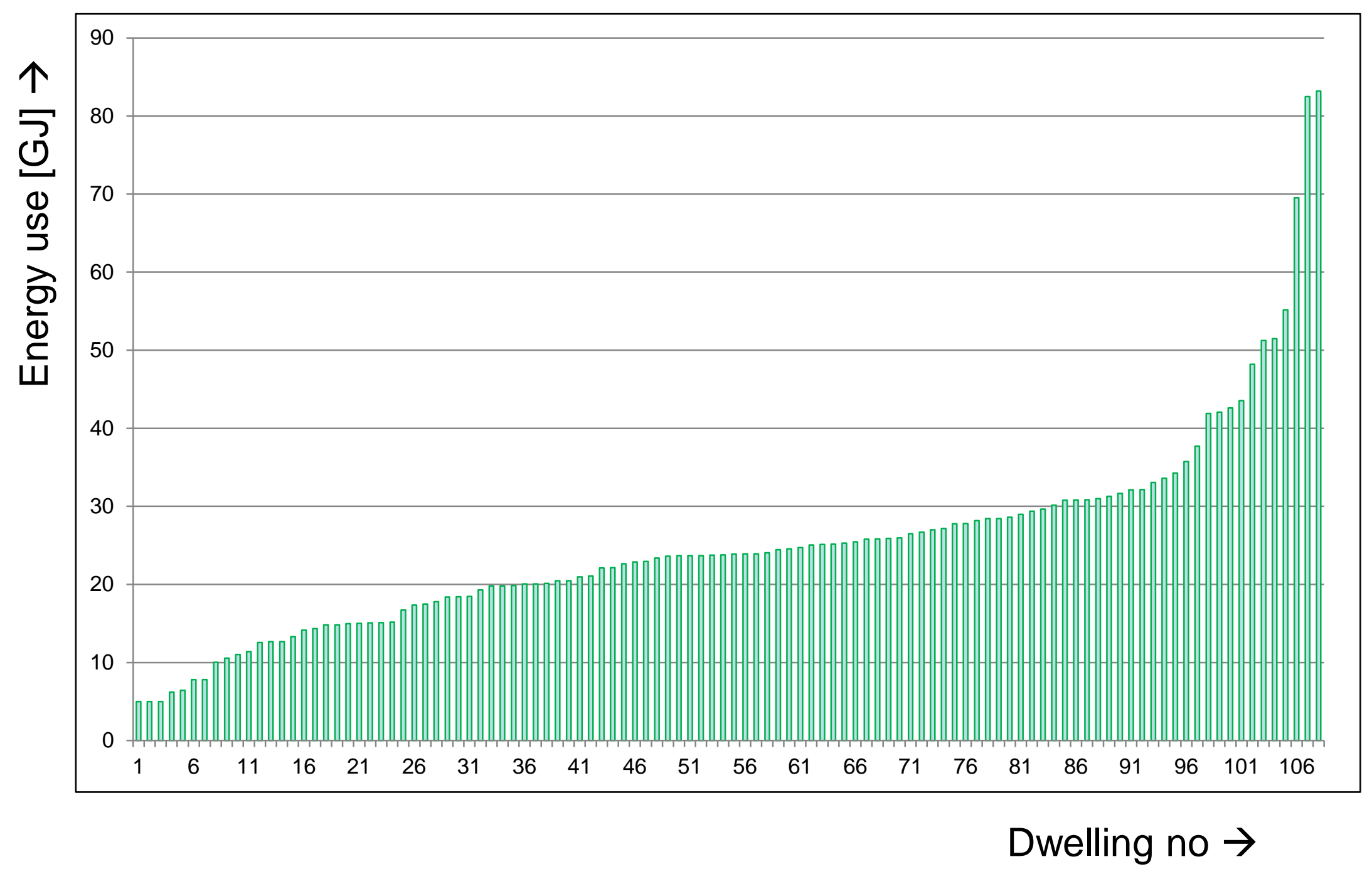


Need for better calculation methodologies?

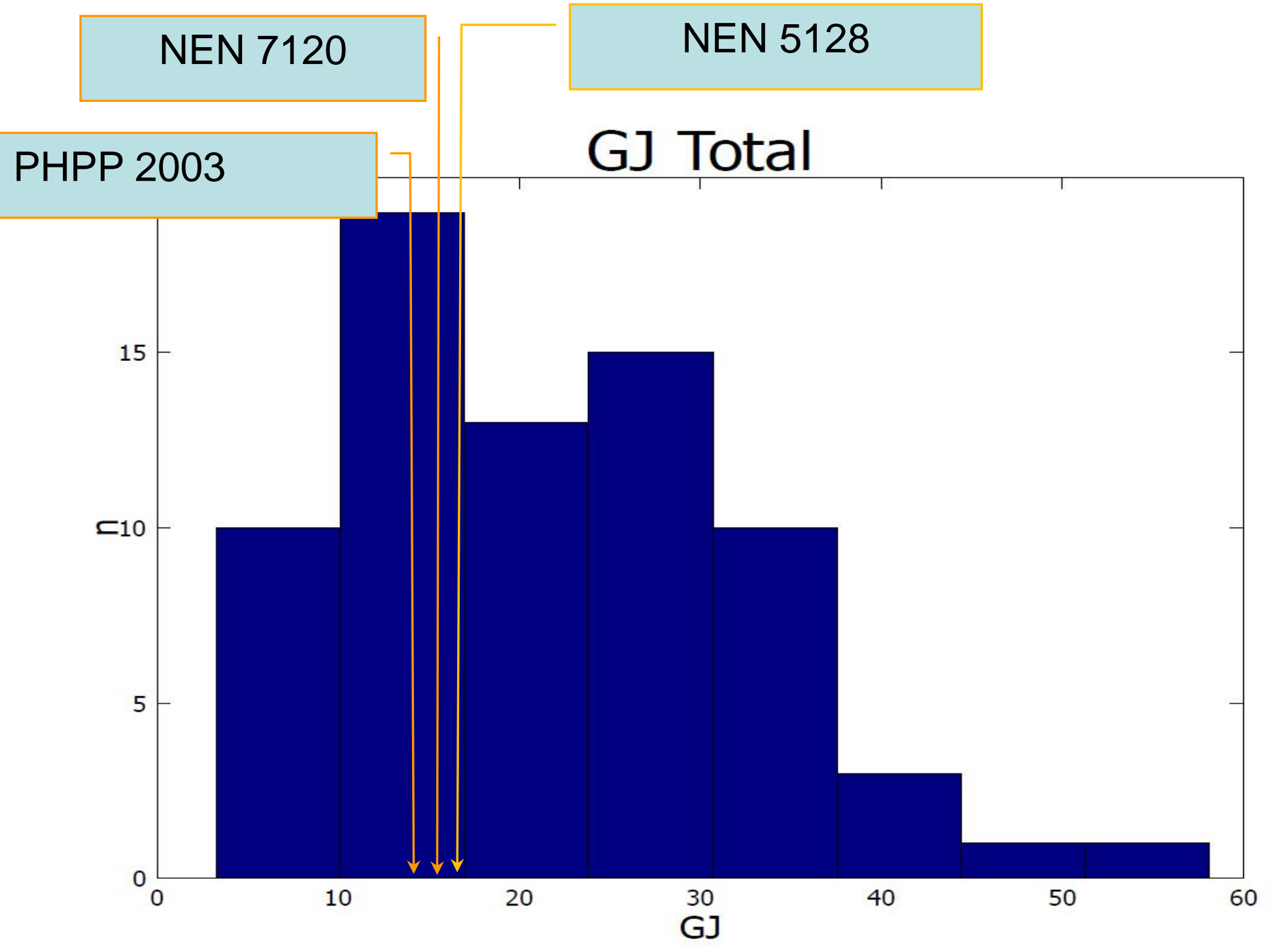




\section{IEA Annex 53 Total energy use in buildings - influencing factors}

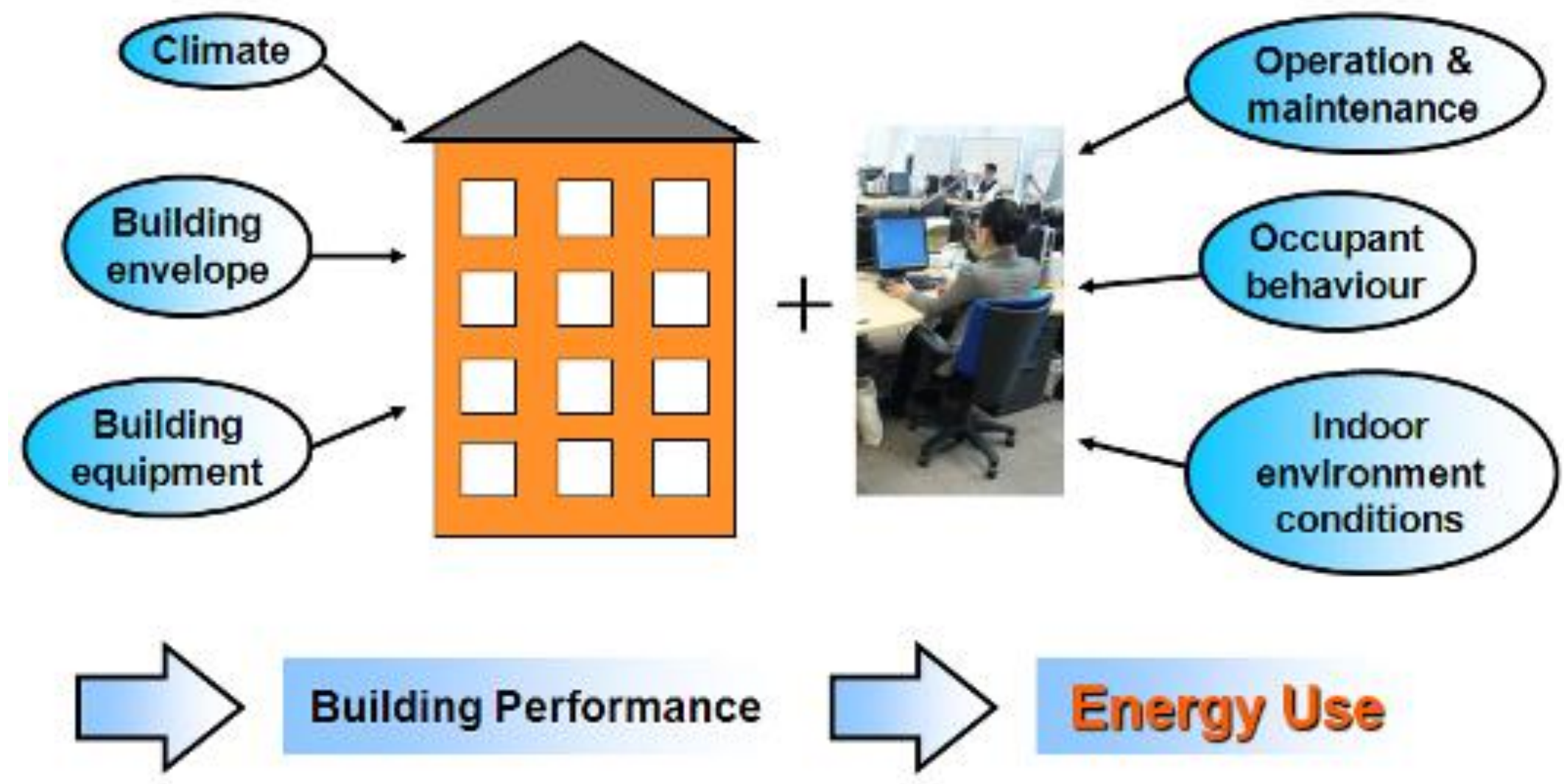




\section{Uncertainties}

1. The energy performance regulations (EPBD)

2. The performance of buildings and equipment

3. The occupant behaviour

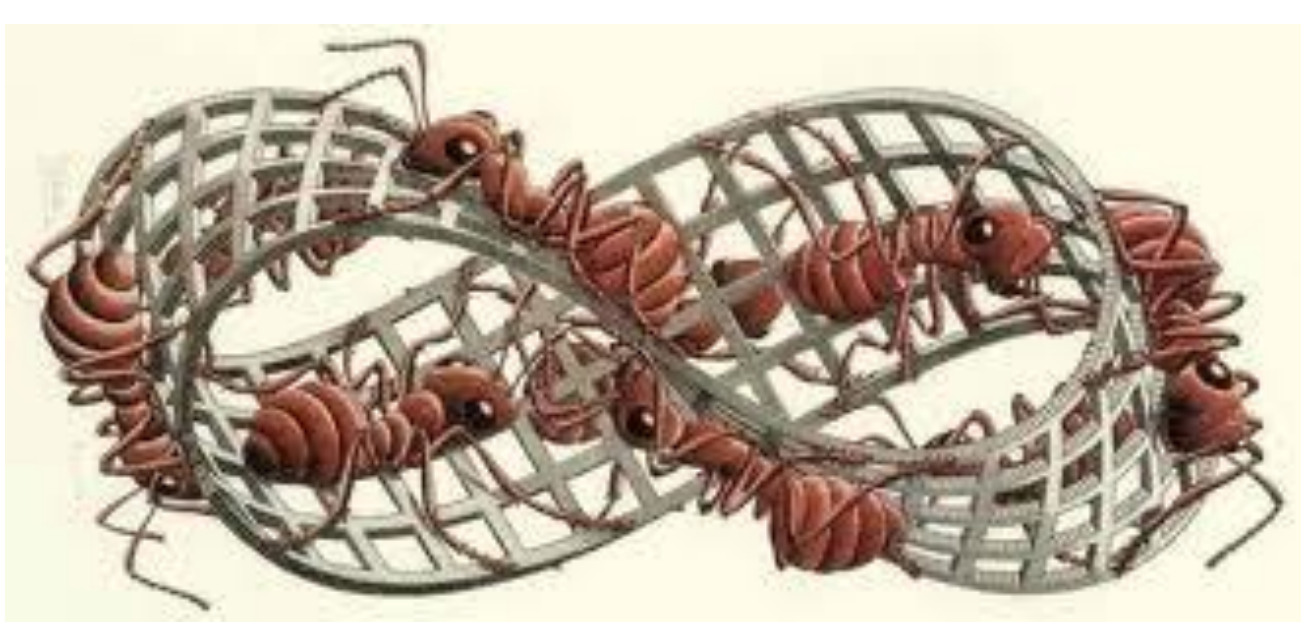




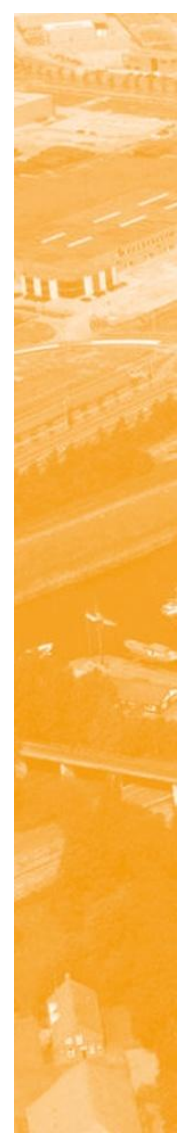

\section{The energy performance regulations (EPBD)}

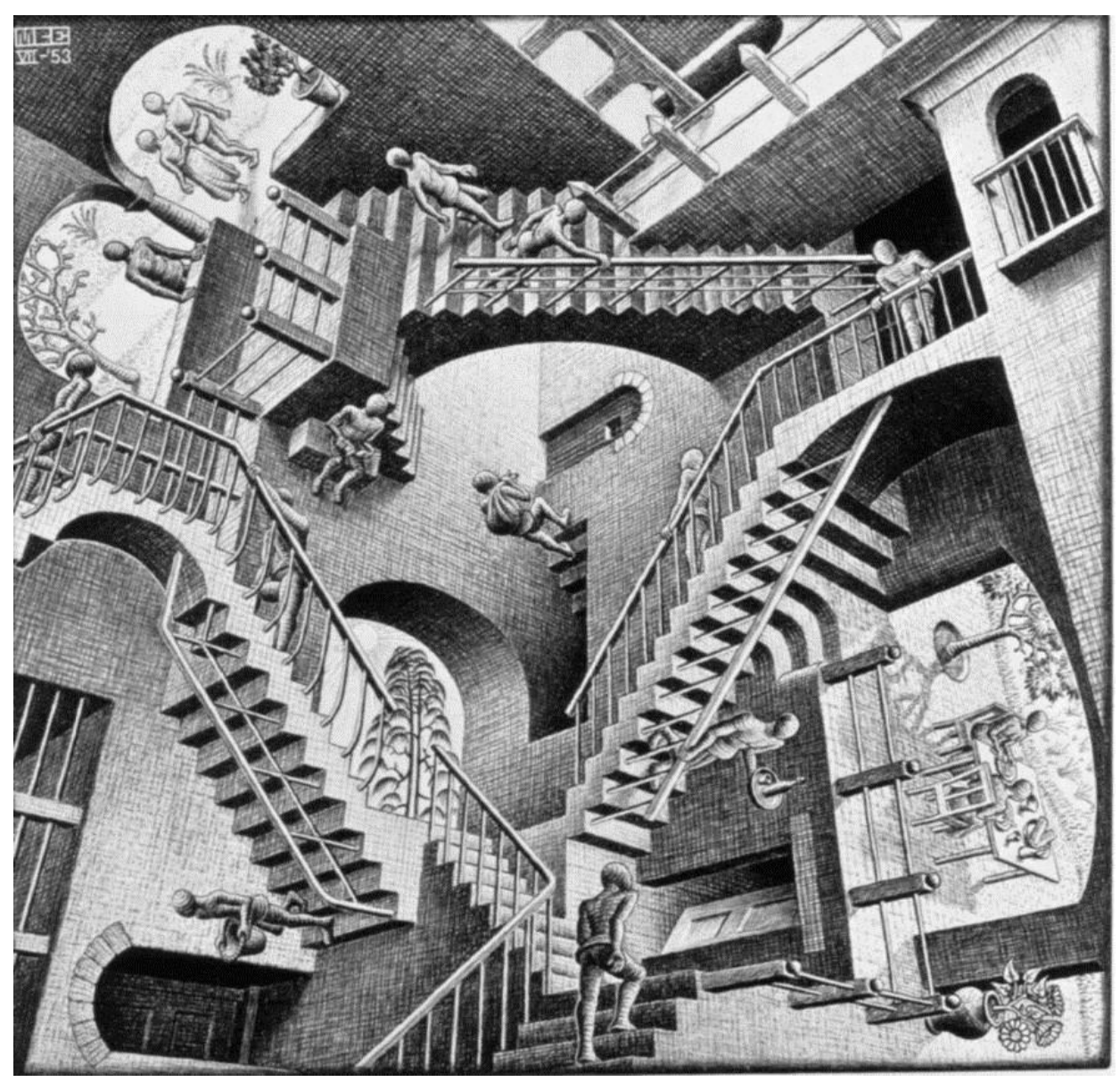




\section{Real energy use versus EPC}

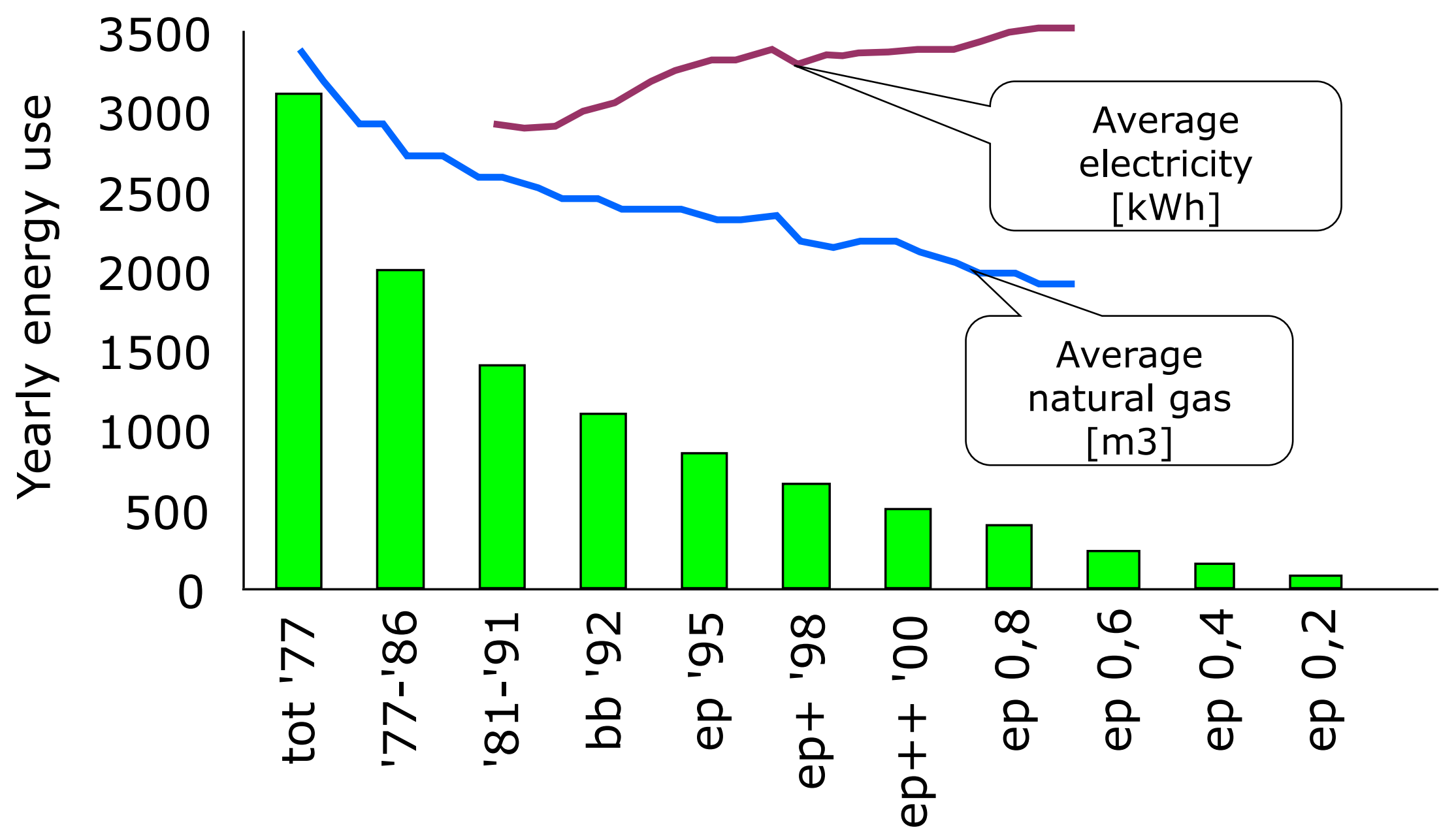




\section{Energy performance calculation}
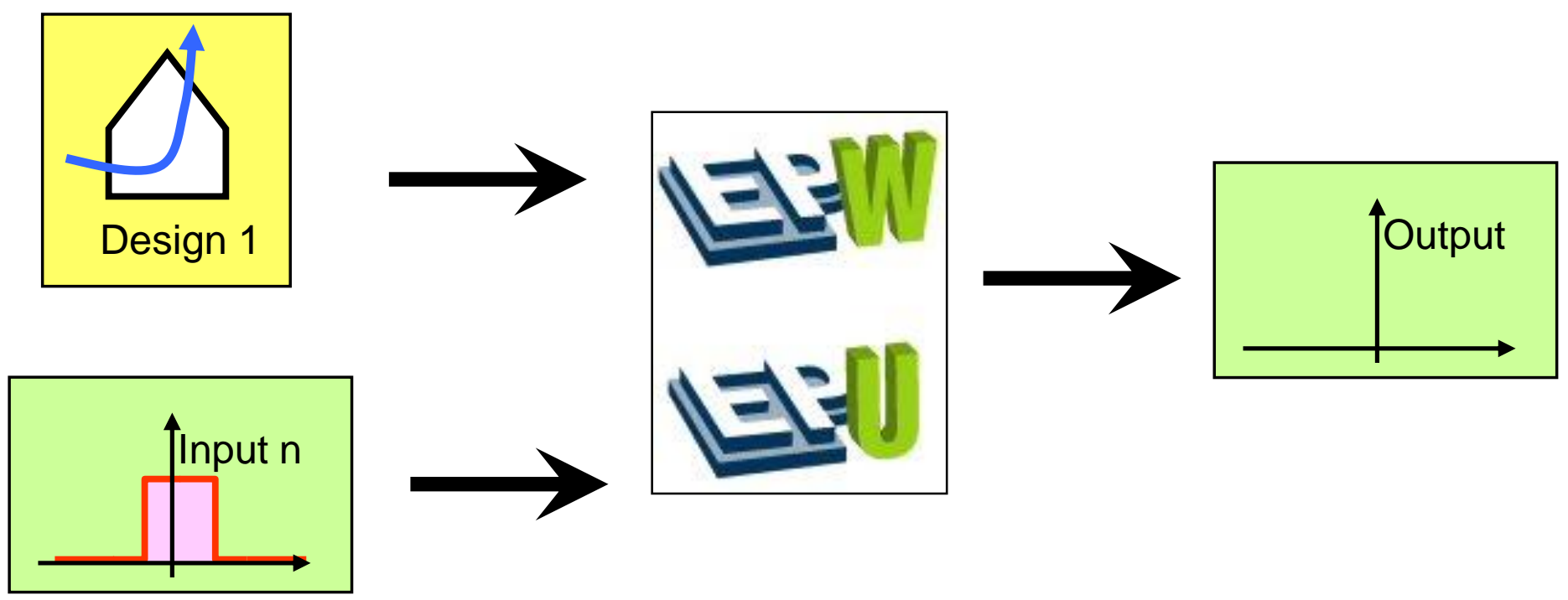

Characteristics

- Related to building regulations

- To obtain a building permit

- Pertaining to public law

- Control at government

- "Labeling" of buildings

- Forfaitair and sometimes arbitrary starting points

- For 1 family type, a climatic condition, etc.

- Output is dimensionless co-efficient 


\section{EPC versus $\mathrm{CO}_{2}$-reduction}

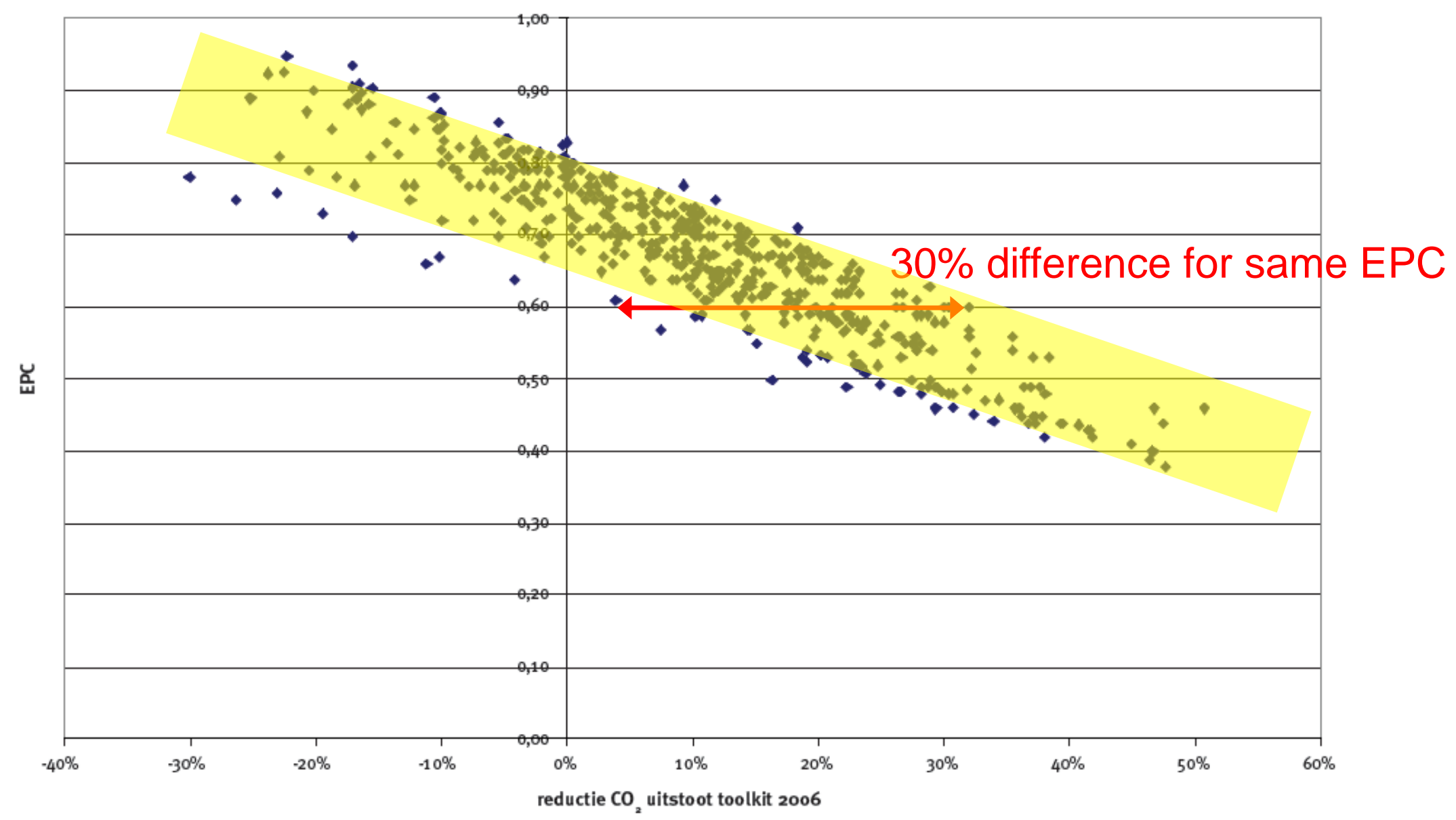

Prestaties van verschillende energieconcepten. Spreiding in $\mathrm{CO}_{2}$-reductie bij een EPC waarde 


\section{Real energy use compared to EPC}

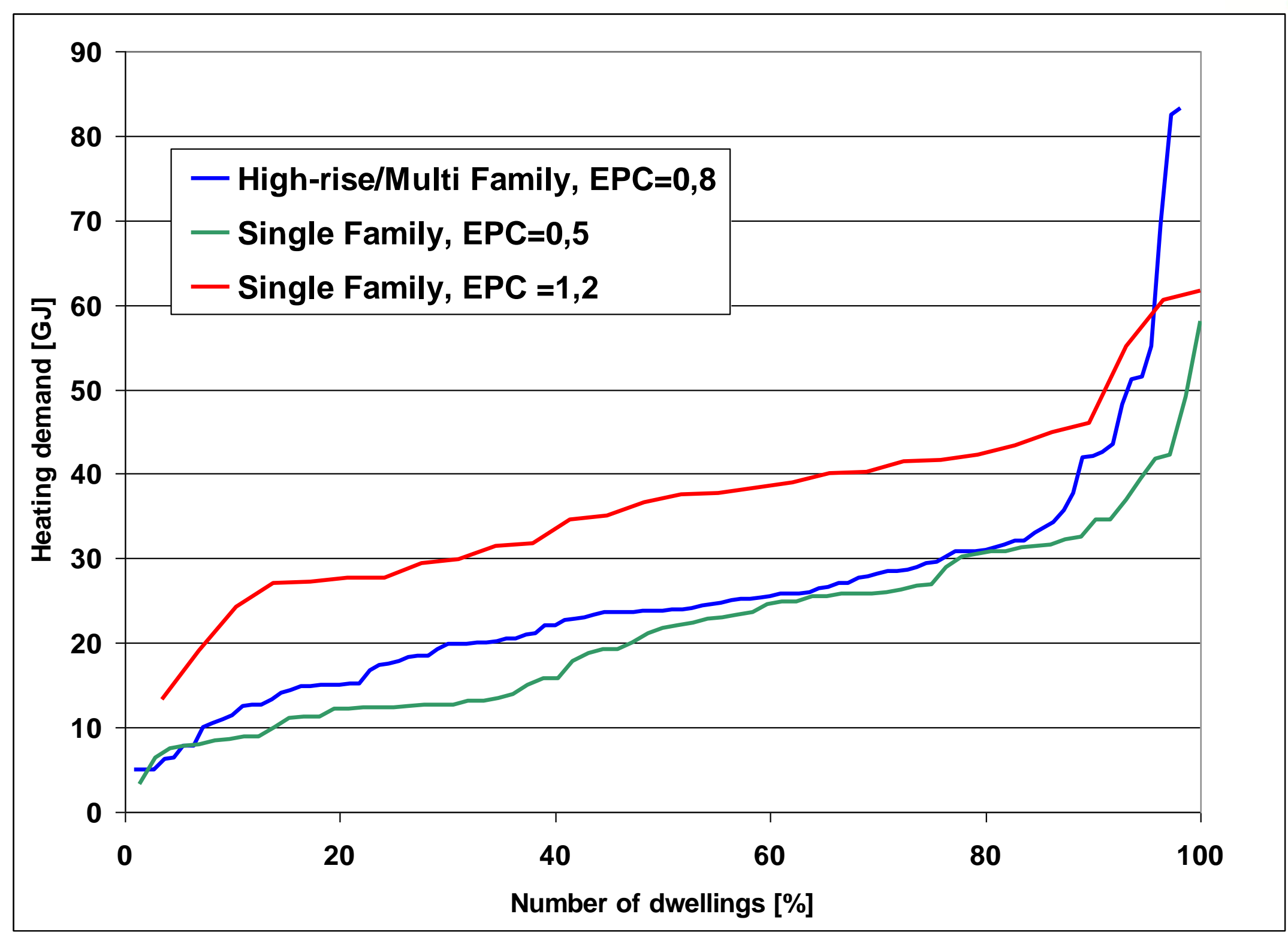


Performance calculations Hybrid ventilation school building

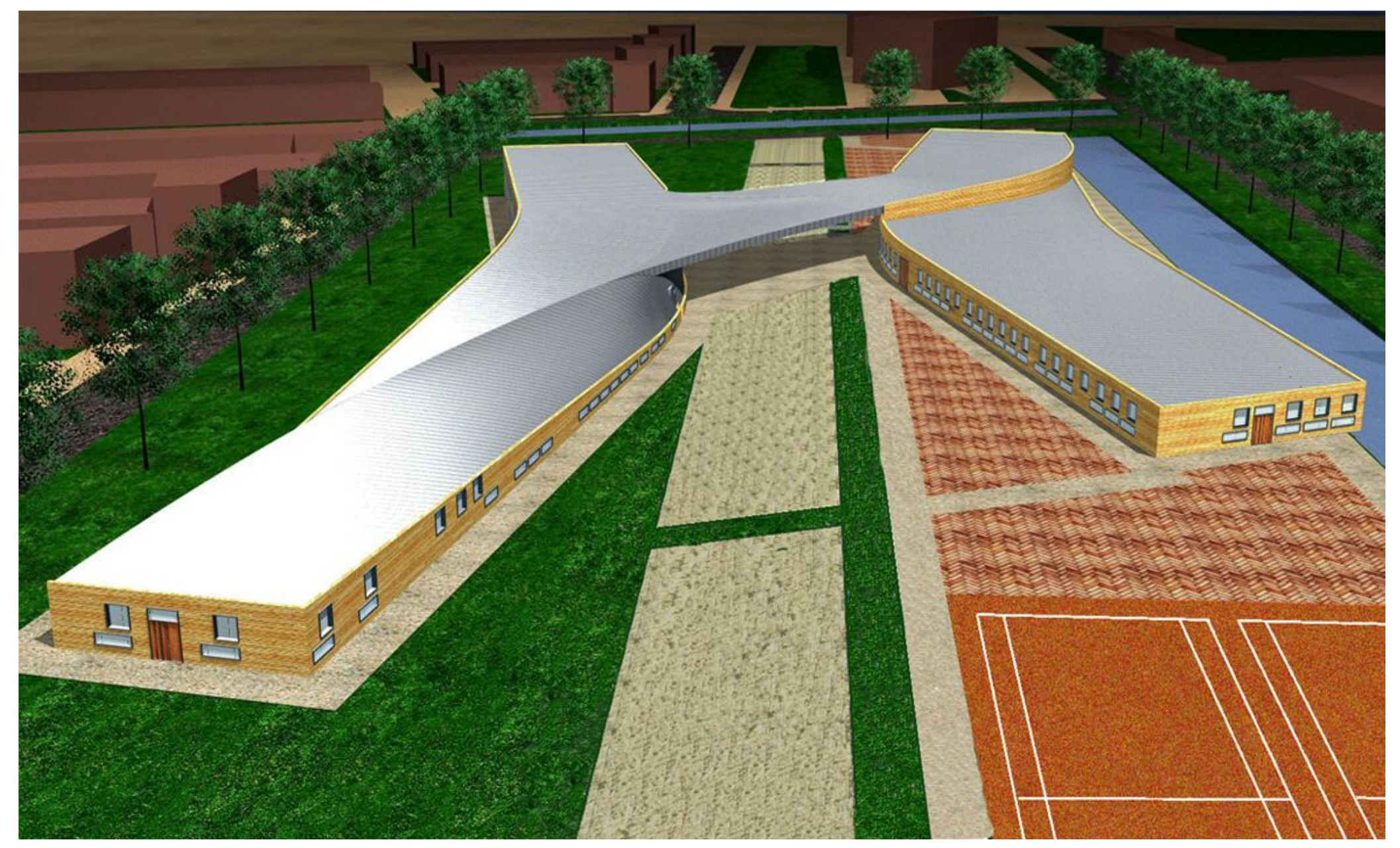




\section{Boundary conditions for Performance Index}

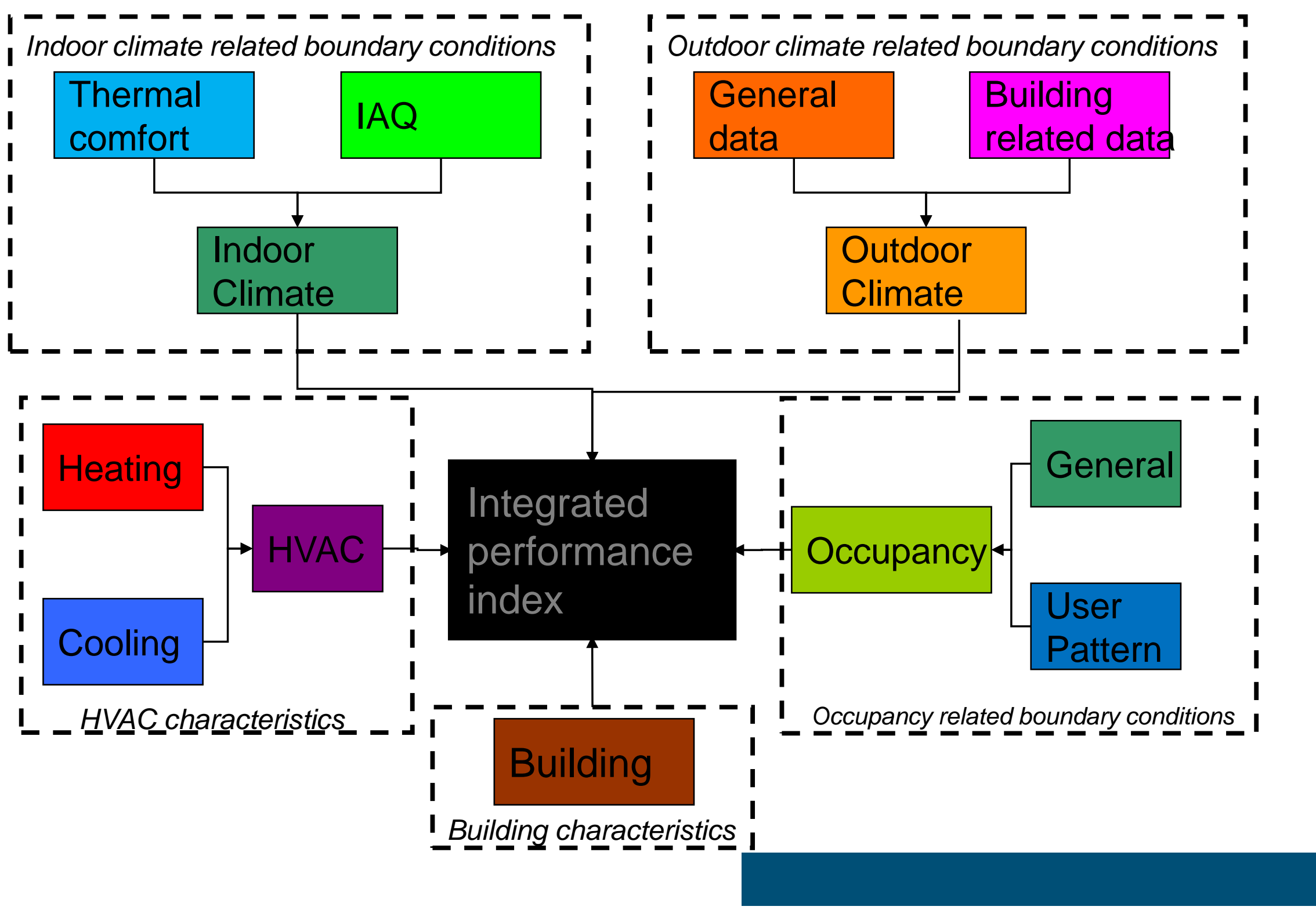




\section{Energy performance coefficient}

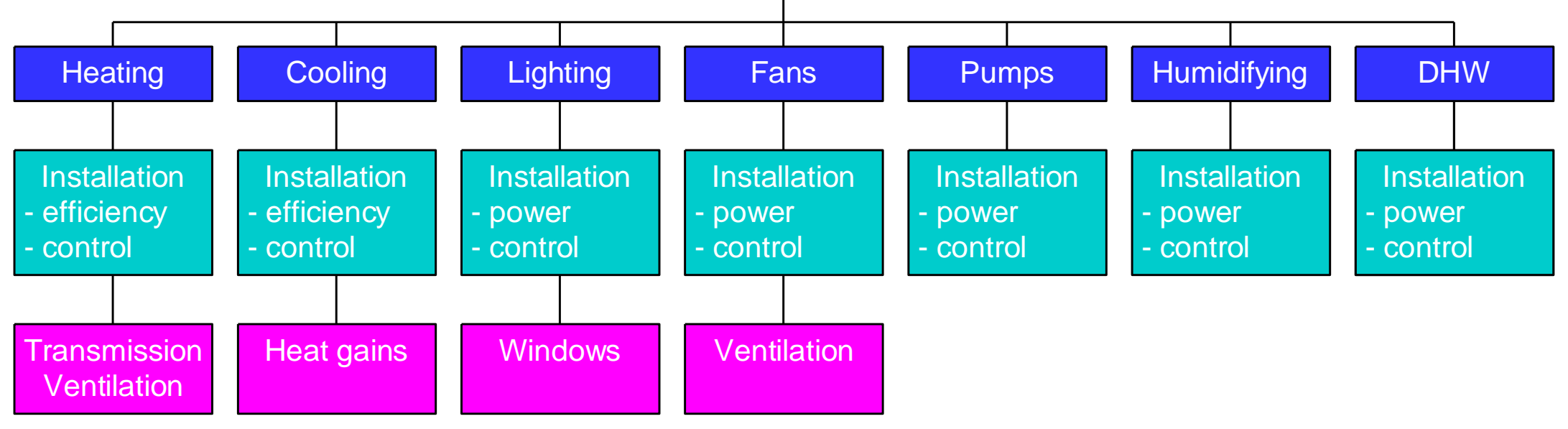

\begin{tabular}{l|l|}
\hline Measure & Calculation \\
\hline$U_{\text {floor }}\left[\mathrm{W} / \mathrm{m}^{2} \mathrm{~K}\right]$ & 0,31 \\
$U_{\text {roof }}\left[\mathrm{W} / \mathrm{m}^{2} \mathrm{~K}\right]$ & 0,37 \\
$U_{\text {facade }}\left[\mathrm{W} / \mathrm{m}^{2} \mathrm{~K}\right]$ & 0,37 \\
$U_{\text {window }}\left[\mathrm{W} / \mathrm{m}^{2} \mathrm{~K}\right]$ & 1,8 \\
Shading & No \\
Infiltration $\mathrm{q}_{\mathrm{v} ; 10}\left[\mathrm{dm}^{3} / \mathrm{s}\right]$ & 200 per $3000 \mathrm{~m}^{3}$ \\
Ventilation & Natural inlet / Mechanical exhaust \\
Fan $[\mathrm{kW}]$ & Peff $_{\text {en }}$ \\
Heating production & Gas fired HE-boiler \\
HVAC system & Class I: Mech. Ventilation, radiators \\
DHW production & Gas fired HE-boiler \\
Lighting $\left[\mathrm{W} / \mathrm{m}^{2}\right]$ & 12 HF lighting + local switch \\
$\mathbf{Q}_{\text {pres,tot }} / \mathbf{Q}_{\text {pres,acc }}$ & $\mathbf{0 , 9 8}$ \\
\hline
\end{tabular}




\section{Principle of equivalence}

System does not fulfil the req's but has the same performance
System cannot be tested according standardised test method

\section{Escape route}

specified in Building decree

\section{Equivalence performance approval}




\section{Equivalent EPC}

Consumption Prim. energy [MJ] Alteration [\%] Prim. energy [MJ] Mechvent Hybvent

\begin{tabular}{|l|r|r|r}
\hline Heating & $2,441,597$ & -24.3 & $1,848,289$ \\
\hline Fans & 228,000 & -77.9 & 50,388 \\
\hline Lighting & $1,229,738$ & & $1,229,738$ \\
\hline Pumps & 67,987 & & 67,987 \\
\hline DHW & 157,397 & 157,397 \\
\hline Qpres;total & $4,124,719$ & $3,353,799$ \\
\hline Qpres;acc & $4,193,046$ & $4,193,046$ \\
\hline Qpt/Qpa & $\mathbf{0 . 9 8}$ & $\mathbf{0 . 8 0}$ \\
\hline
\end{tabular}

Hybrid ventilation: $-20 \%$ 


\section{Boundary conditions}

- Fixed parameters

$\Rightarrow$ Building characteristics

- Thermal insulation

- Windows

- Air tightness

- Thermal mass

$\Rightarrow$ Outdoor climate (SRY)

$\Rightarrow$ Internal temperature
- Variable parameters

$\Rightarrow$ Pressure distribution

$\Rightarrow$ Occupancy pattern

$\Rightarrow \mathrm{CO}_{2}$ production

$\Rightarrow$ Acceptable IAQ $\left(\mathrm{CO}_{2}\right)$

$\Rightarrow$ Nighttemperature control

$\Rightarrow$ Internal heat production 


\section{Pressure distribution}

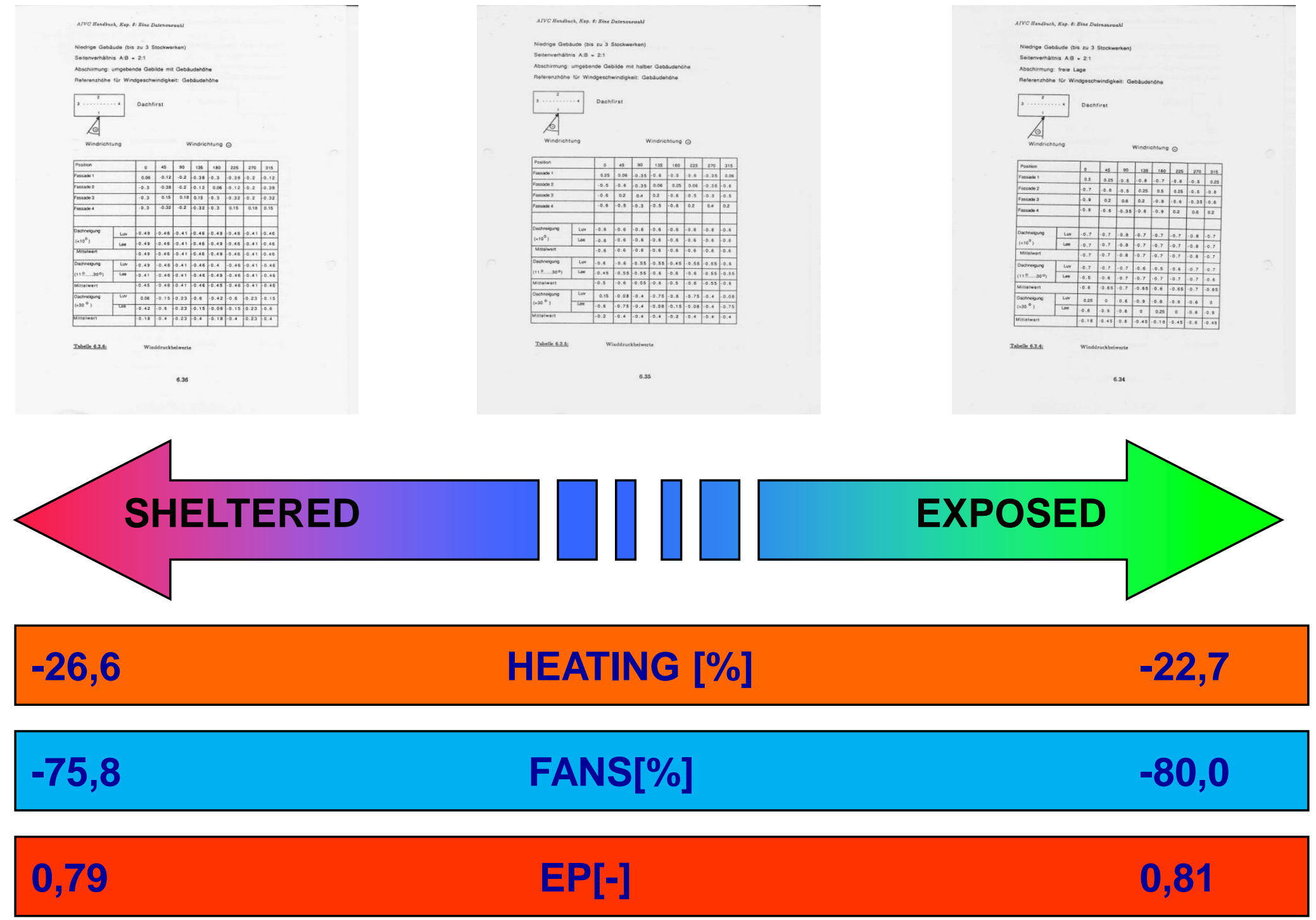




\section{Acceptable $\mathrm{CO}_{2}$ concentration}
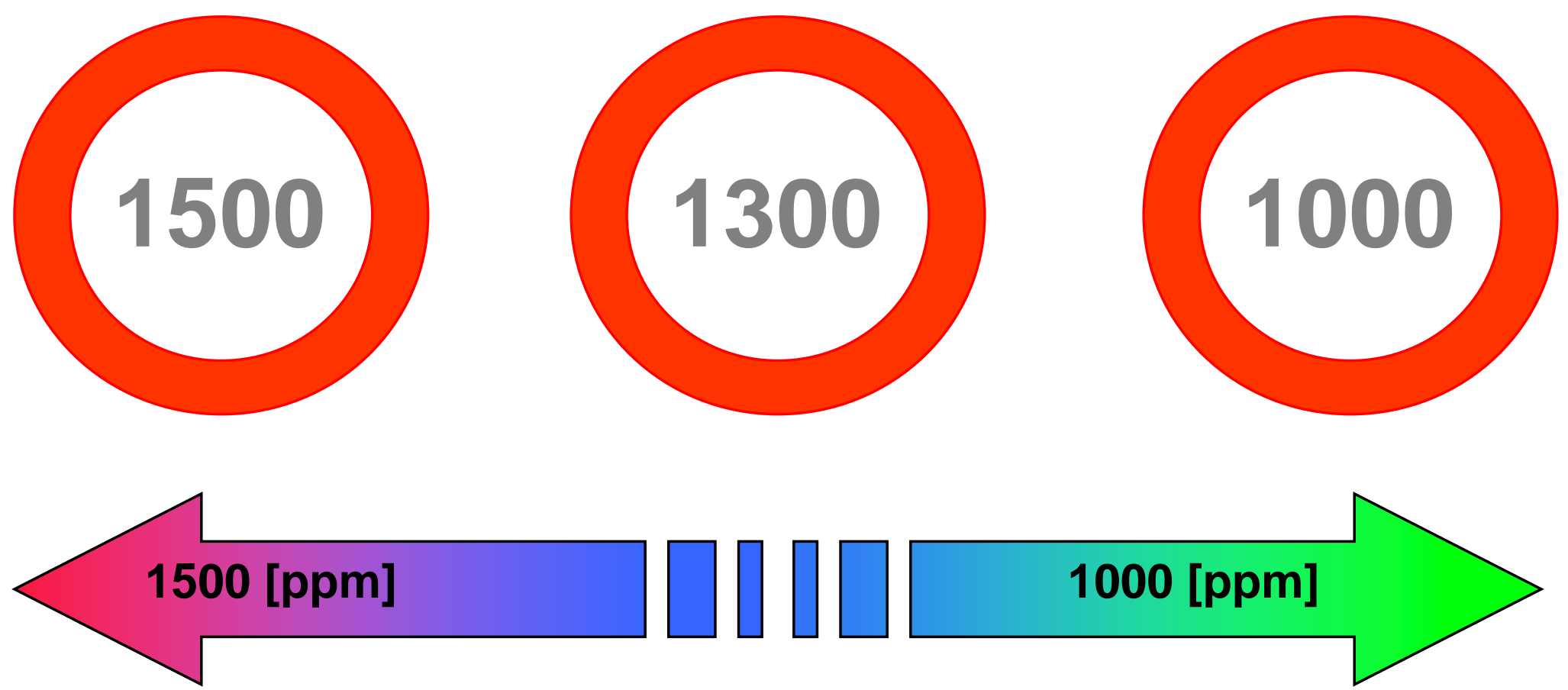

\begin{tabular}{|llc|}
\hline$-26,9$ & HEATING [\%] & $-22,4$ \\
\hline$-87,3$ & FANS[\%] & $-48,9$ \\
\hline 0,78 & EP[-] & 0,83 \\
\hline
\end{tabular}




\section{$\mathrm{CO}_{2}$ production}

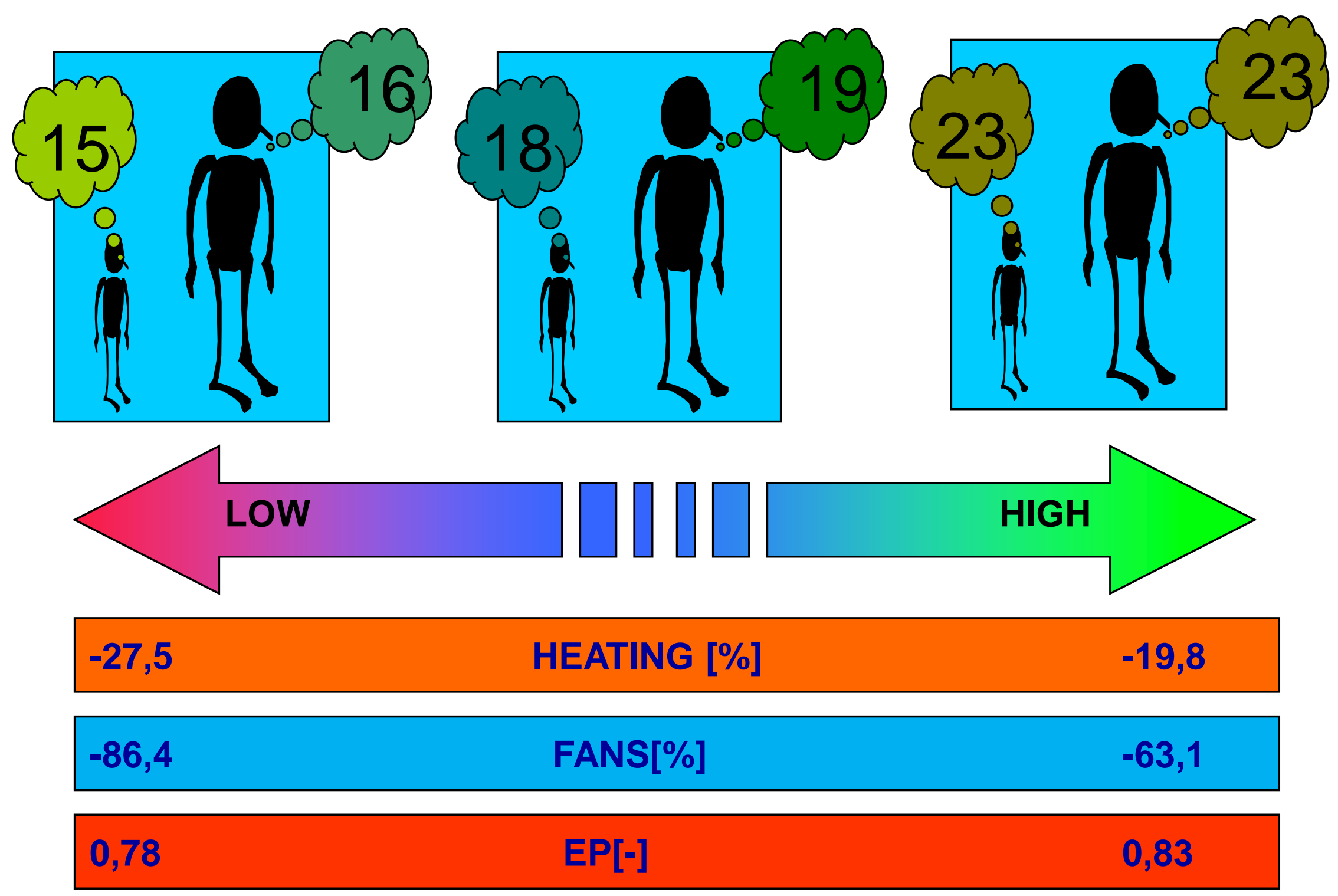




\section{Night temperature control}
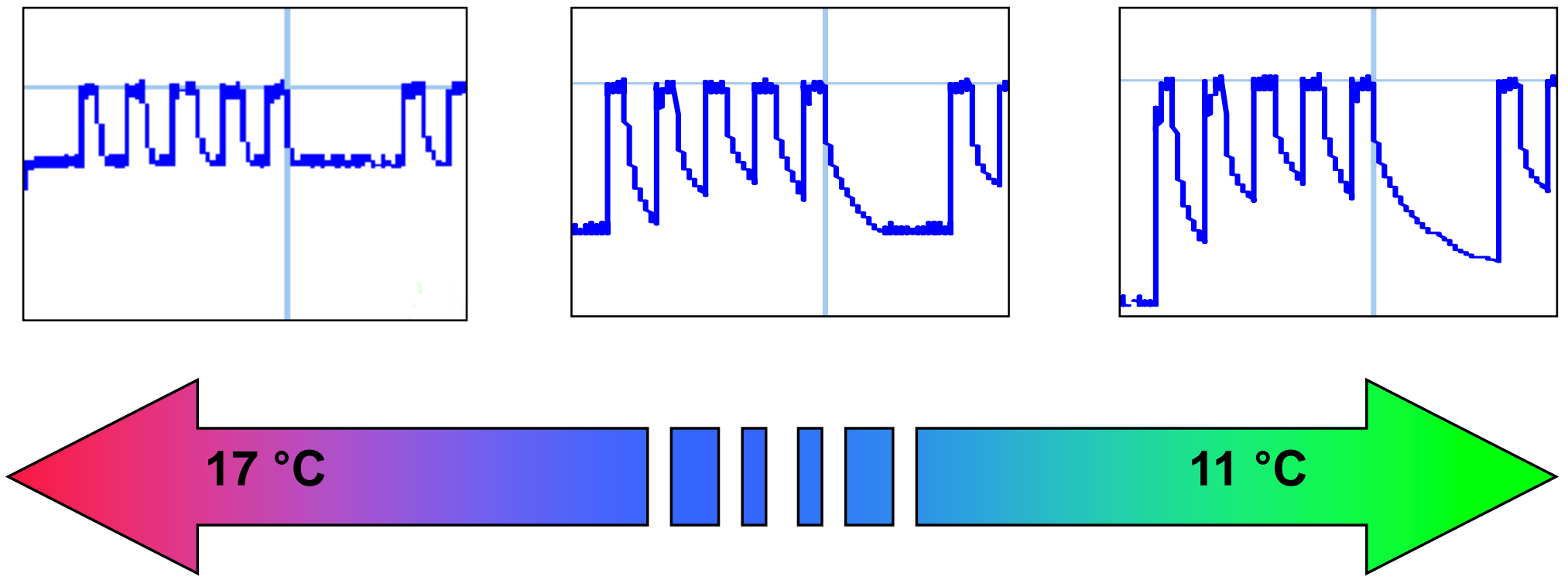

\begin{tabular}{|lcc|}
\hline$-23,1$ & HEATING [\%] & $-24,8$ \\
\hline$-77,9$ & FANS[\%] & $-77,9$ \\
\hline 0,81 & EP[-] & 0,80 \\
\hline
\end{tabular}




\section{Internal heat production}
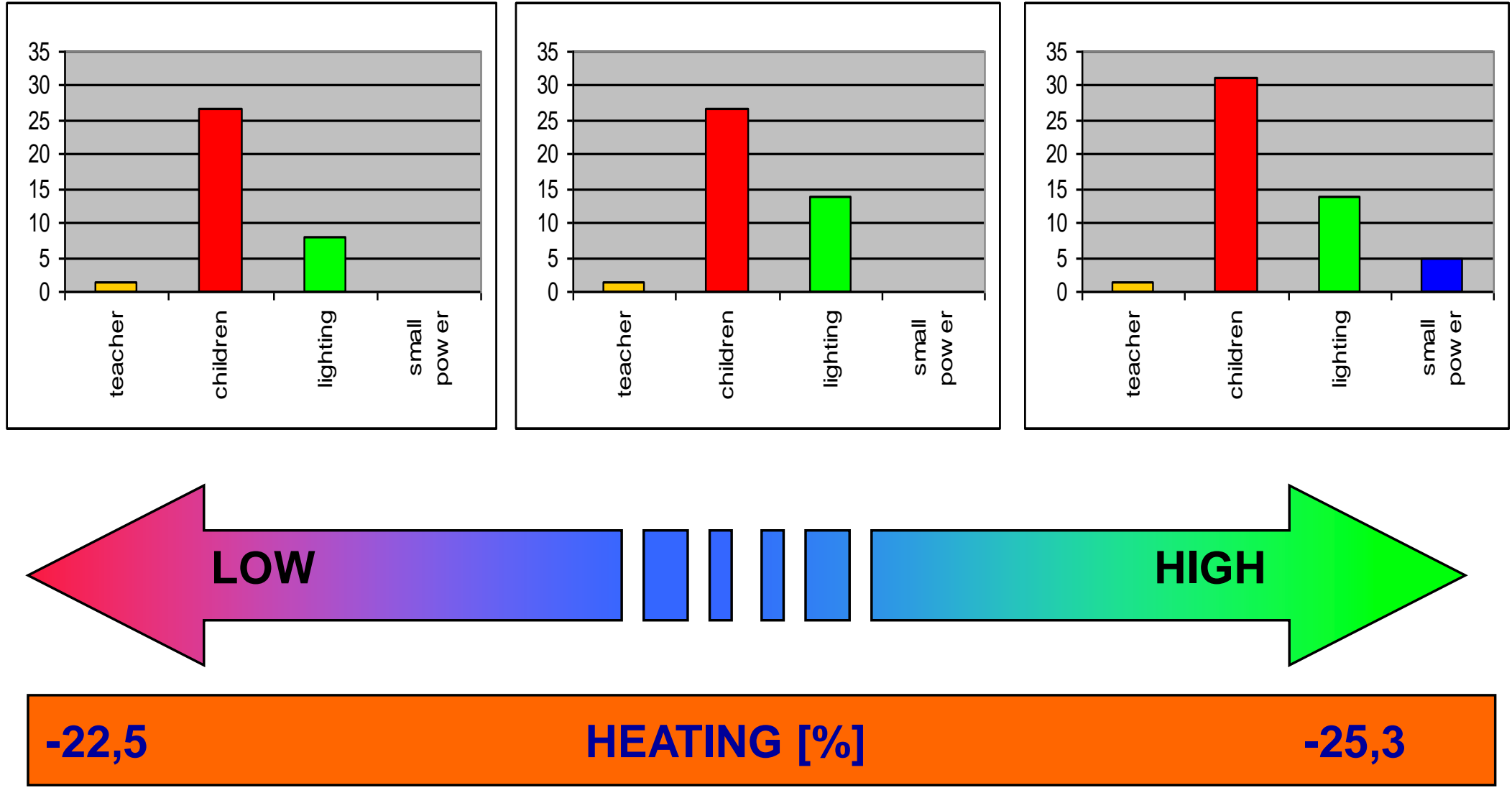

HEATING [\%]

$-25,3$

$-80,1$

FANS[\%]

$-75,8$

0,81

EP[-]

0,80 


\section{Occupancy}
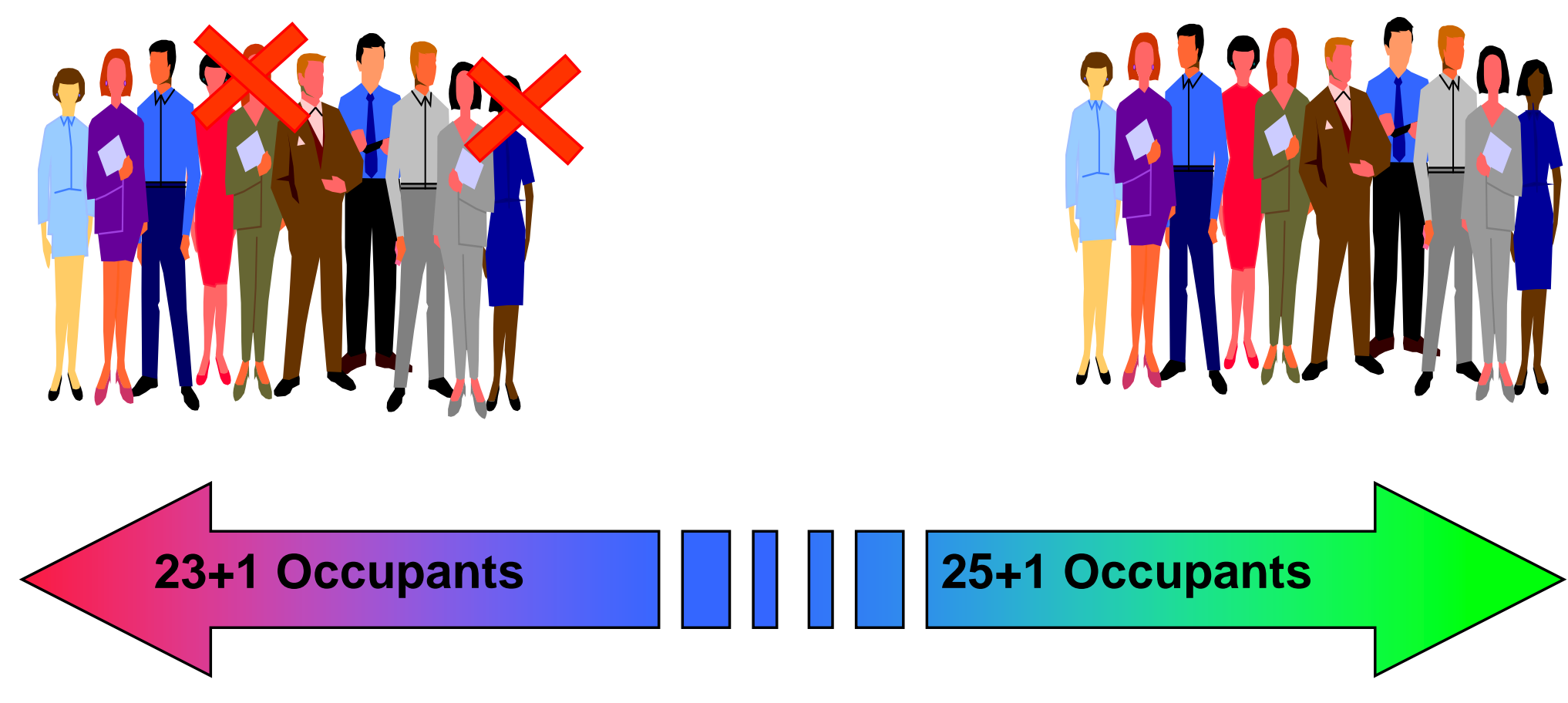

\begin{tabular}{|c|c|c|}
\hline$-23,6$ & HEATING [\%] & $-25,1$ \\
\hline
\end{tabular}

\begin{tabular}{|llc|}
\hline$-80,6$ & FANS[\%] & $-75,2$ \\
\hline 0,80 & EP[-] & 0,80 \\
\hline
\end{tabular}




\section{Results versus medium}

\section{$\%$ Decrease compared with medium}

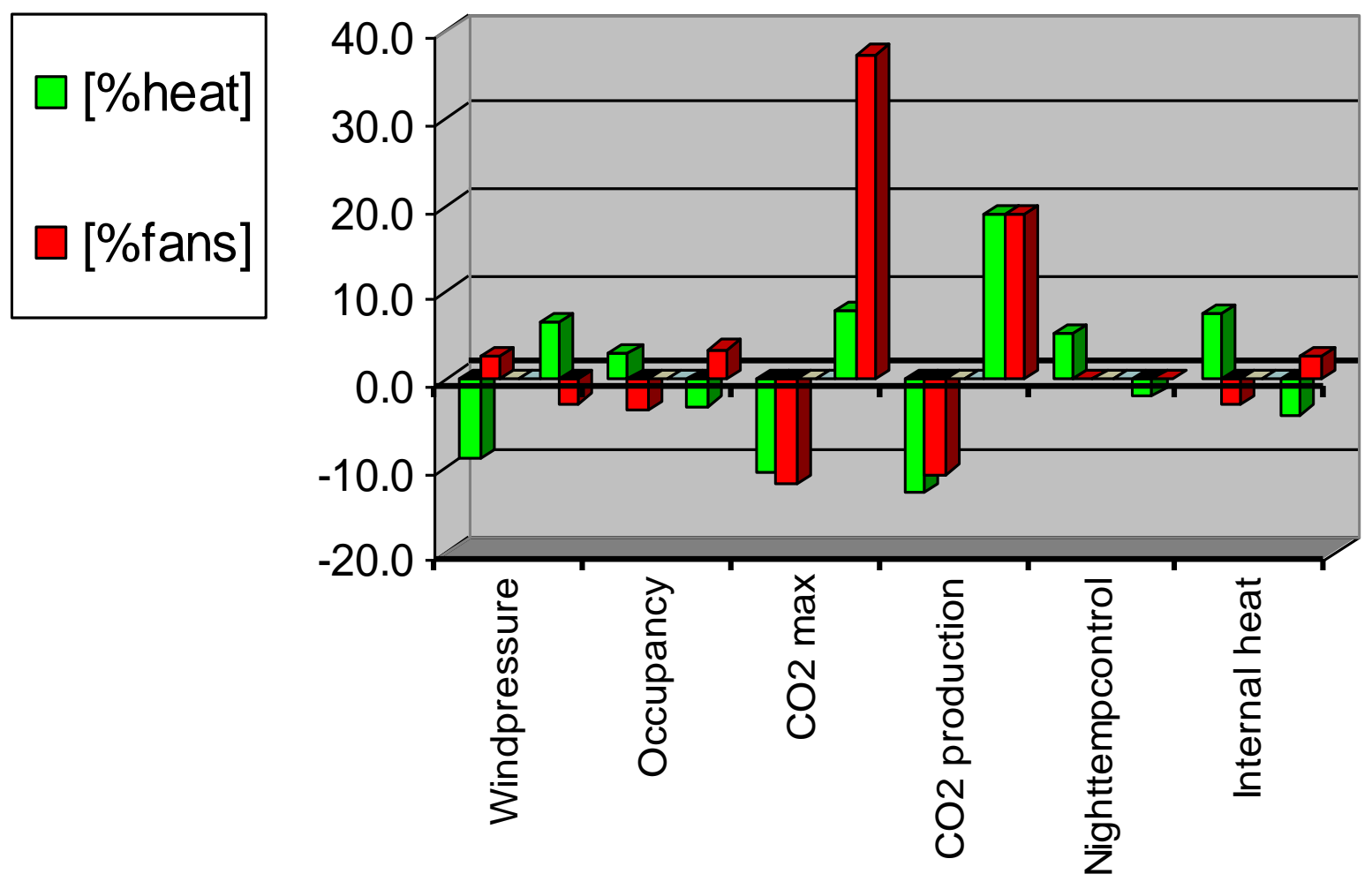




\section{THE EXTREMES}

Evil approach

$\Rightarrow$ Sheltered

$\Rightarrow$ Occupancy high

$\Rightarrow$ Acceptable $\mathrm{CO}_{2}=1500 \mathrm{ppm}$

$\Rightarrow \mathrm{CO}_{2}$ production low

$\Rightarrow$ Nighttemperature low

$\Rightarrow$ Internal heat high
- Decent approach

$\Rightarrow$ Exposed

$\Rightarrow$ Occupancy low

$\Rightarrow$ Acceptable $\mathrm{CO}_{2}=1000 \mathrm{ppm}$

$\Rightarrow \mathrm{CO}_{2}$ production high

$\Rightarrow$ Nighttemperature high

$\Rightarrow$ Internal heat low 


\section{Results of extremes}

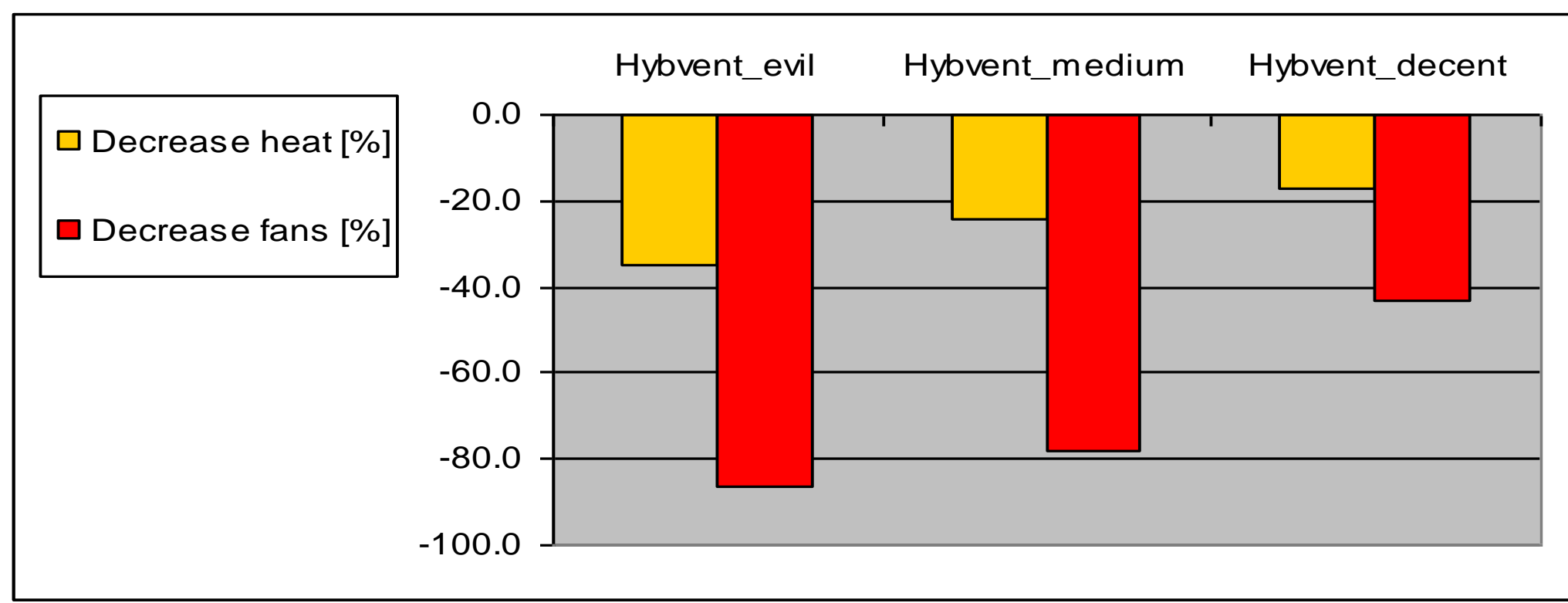

\section{EP [-]}
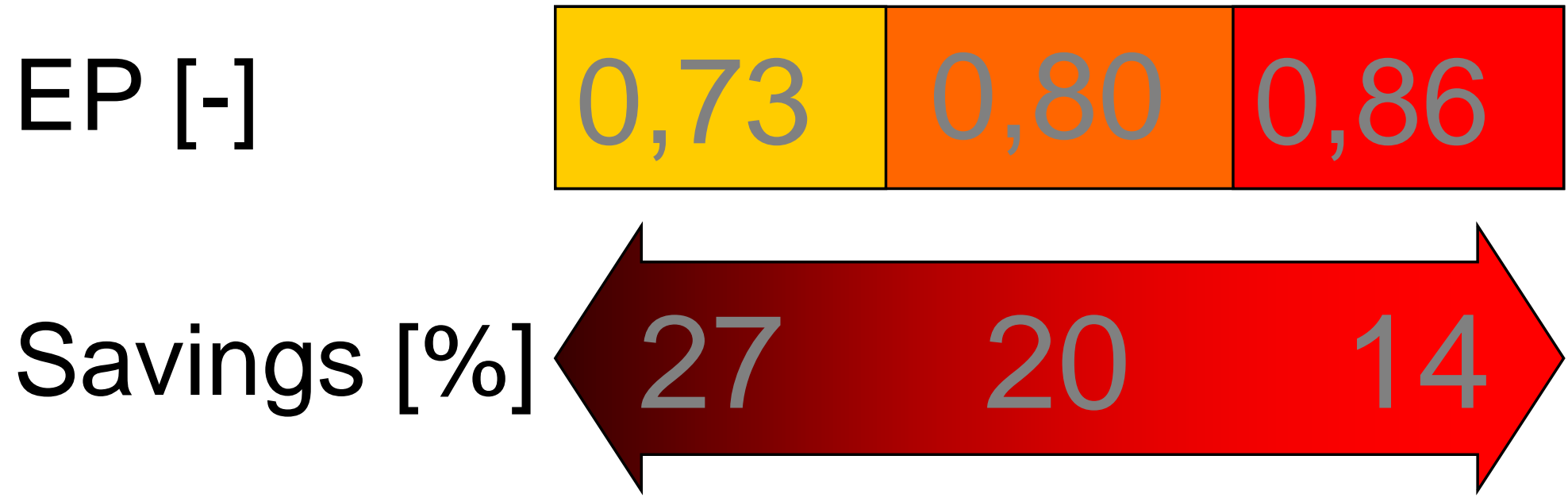
2. The performance of buildings and equipment

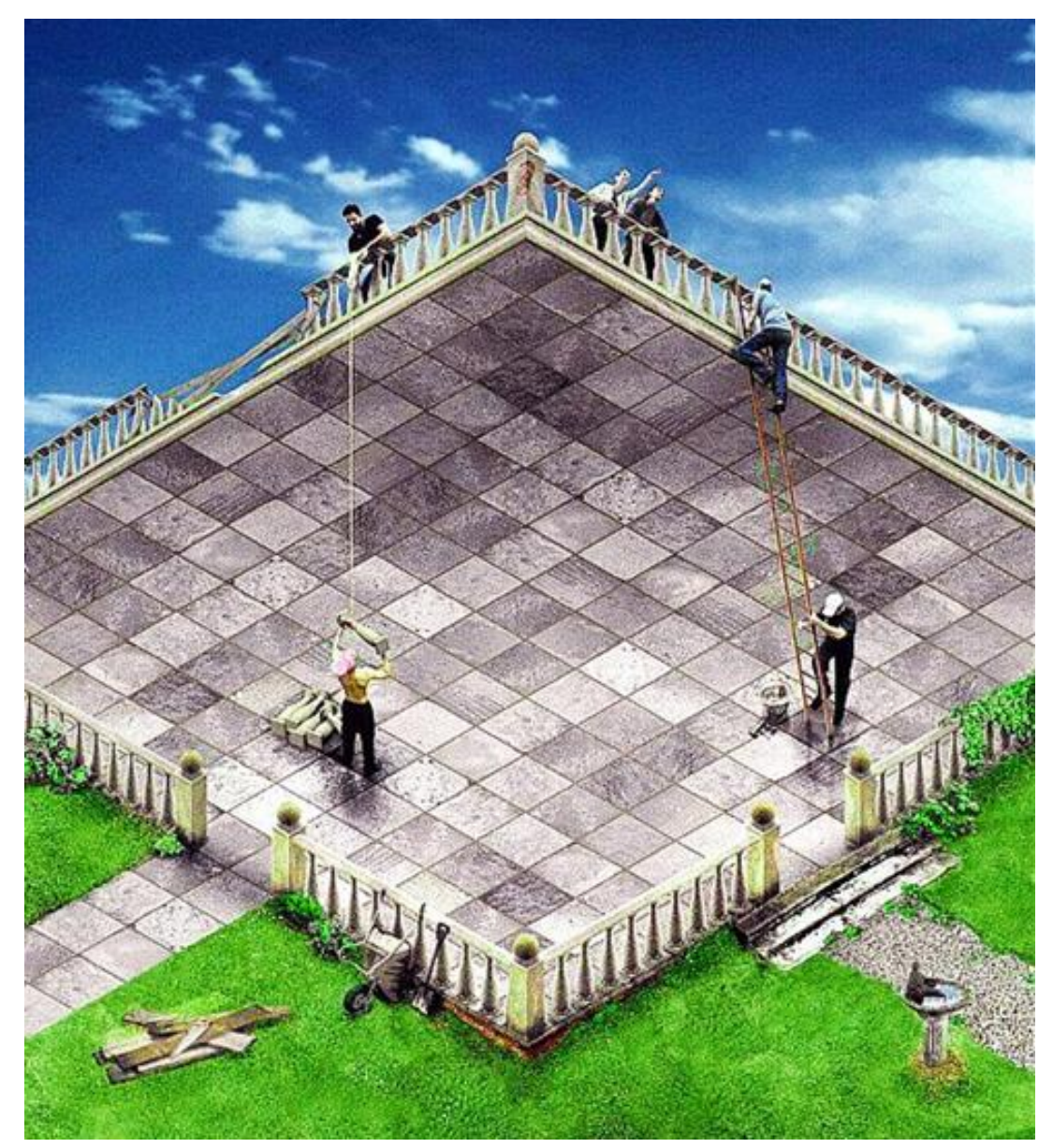




\section{Air tightness of dwellings}

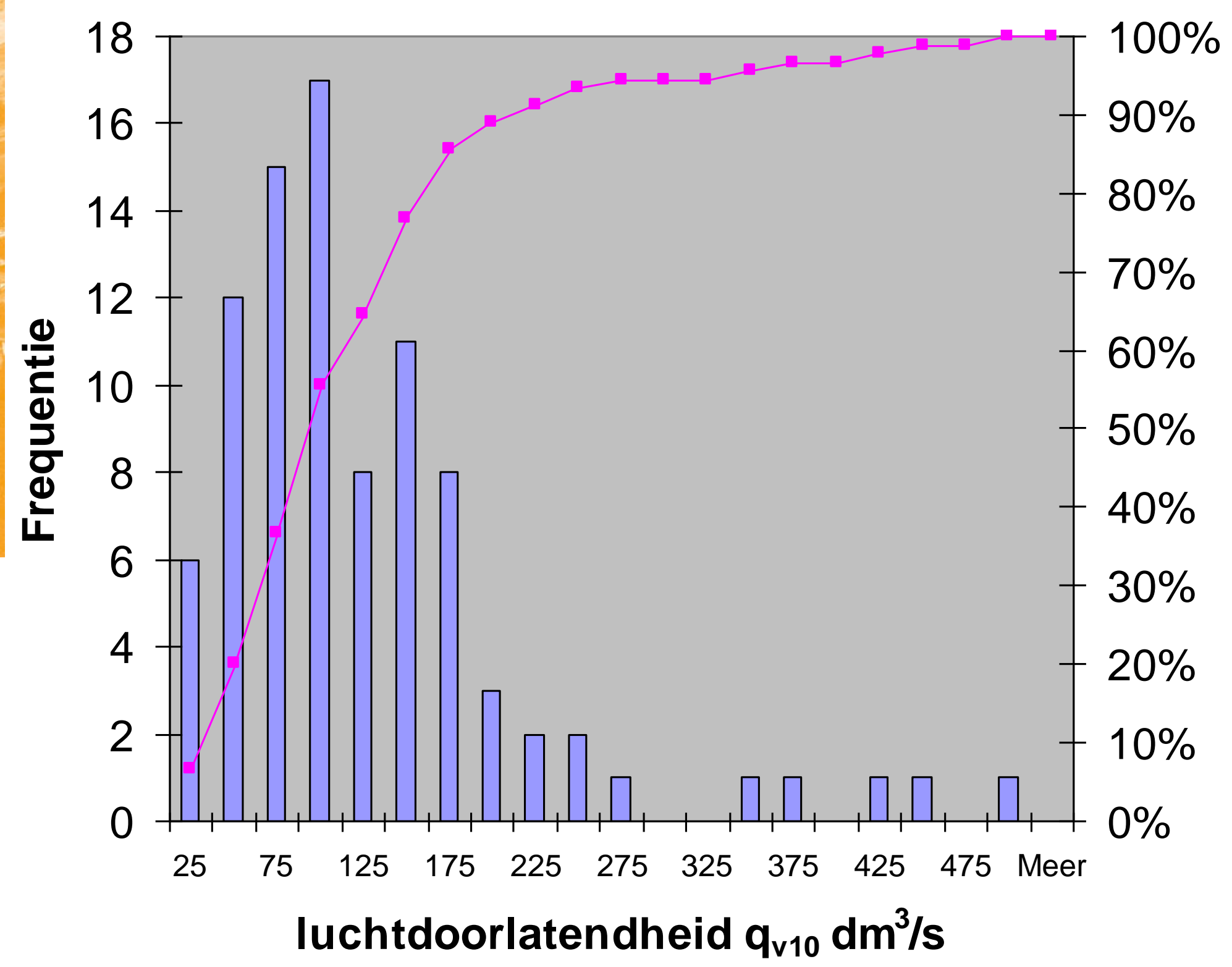




\section{Air tightness before and after renovation}
$\left.q_{i}(10) \mid d m^{3} / s\right]$
spreiding luchtdoorlatendheid voor en na renovatie

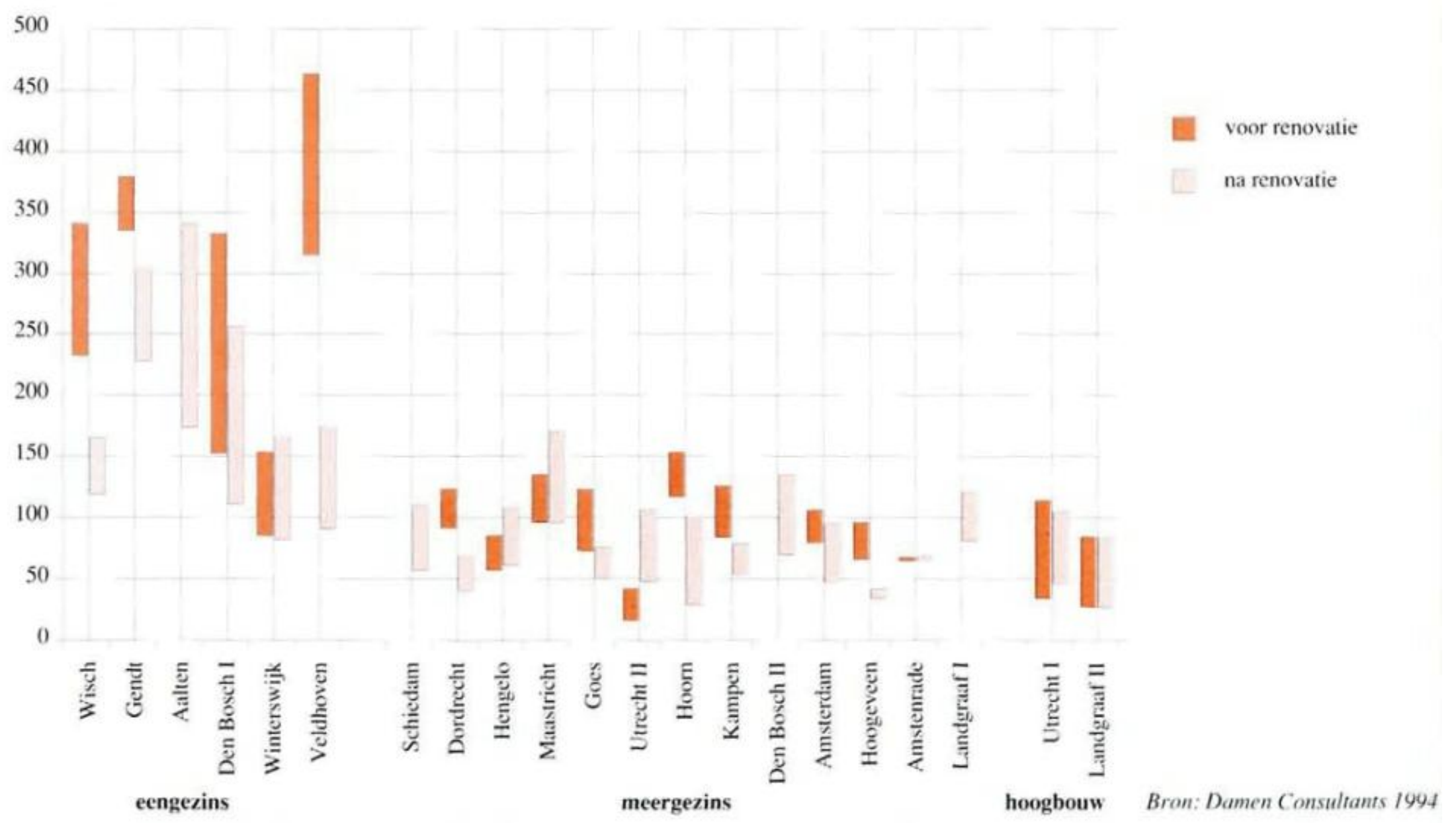


Thermal insulation

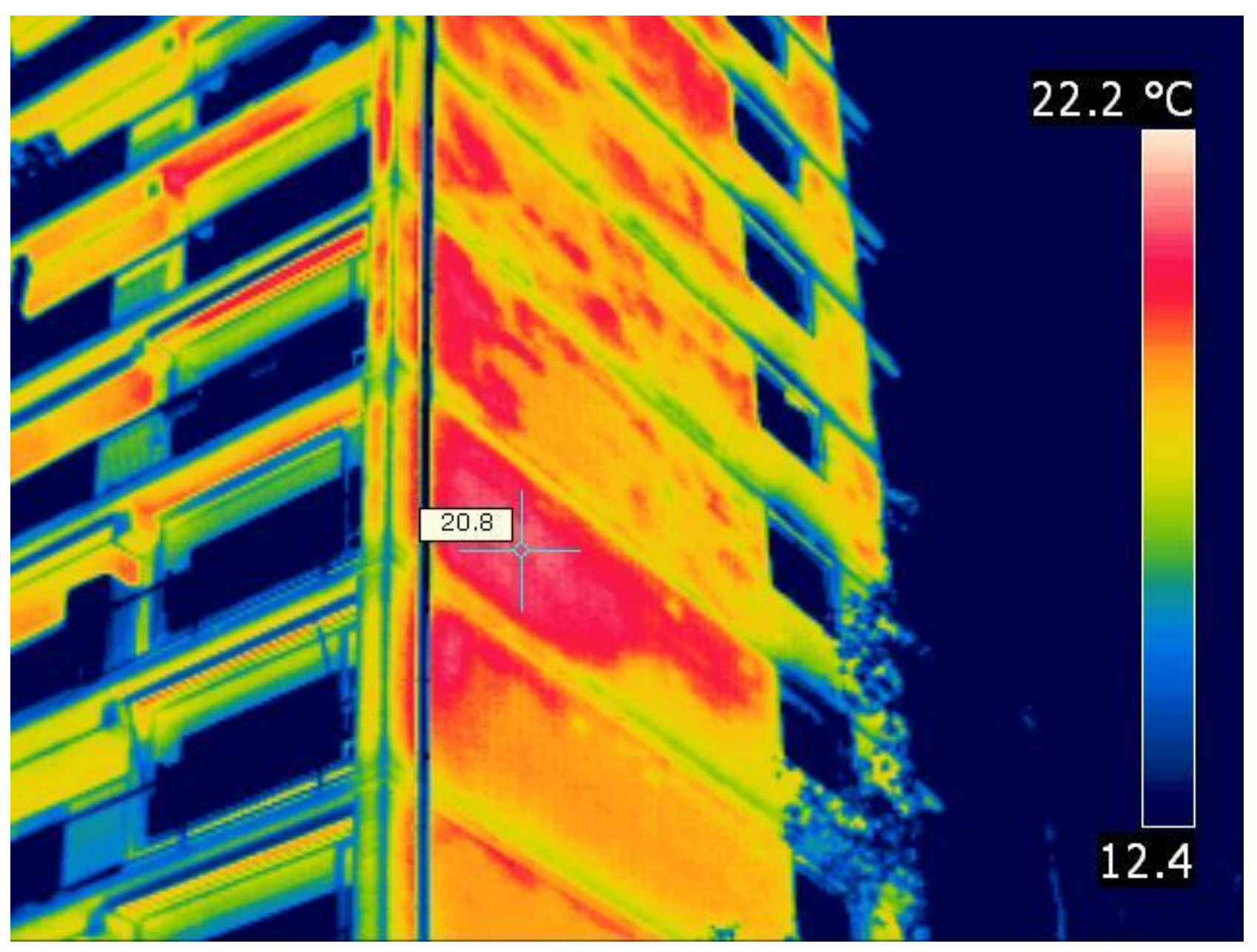


Positioning advanced ventilation in relation to the (Dutch) EPBD

Improvement EPC >

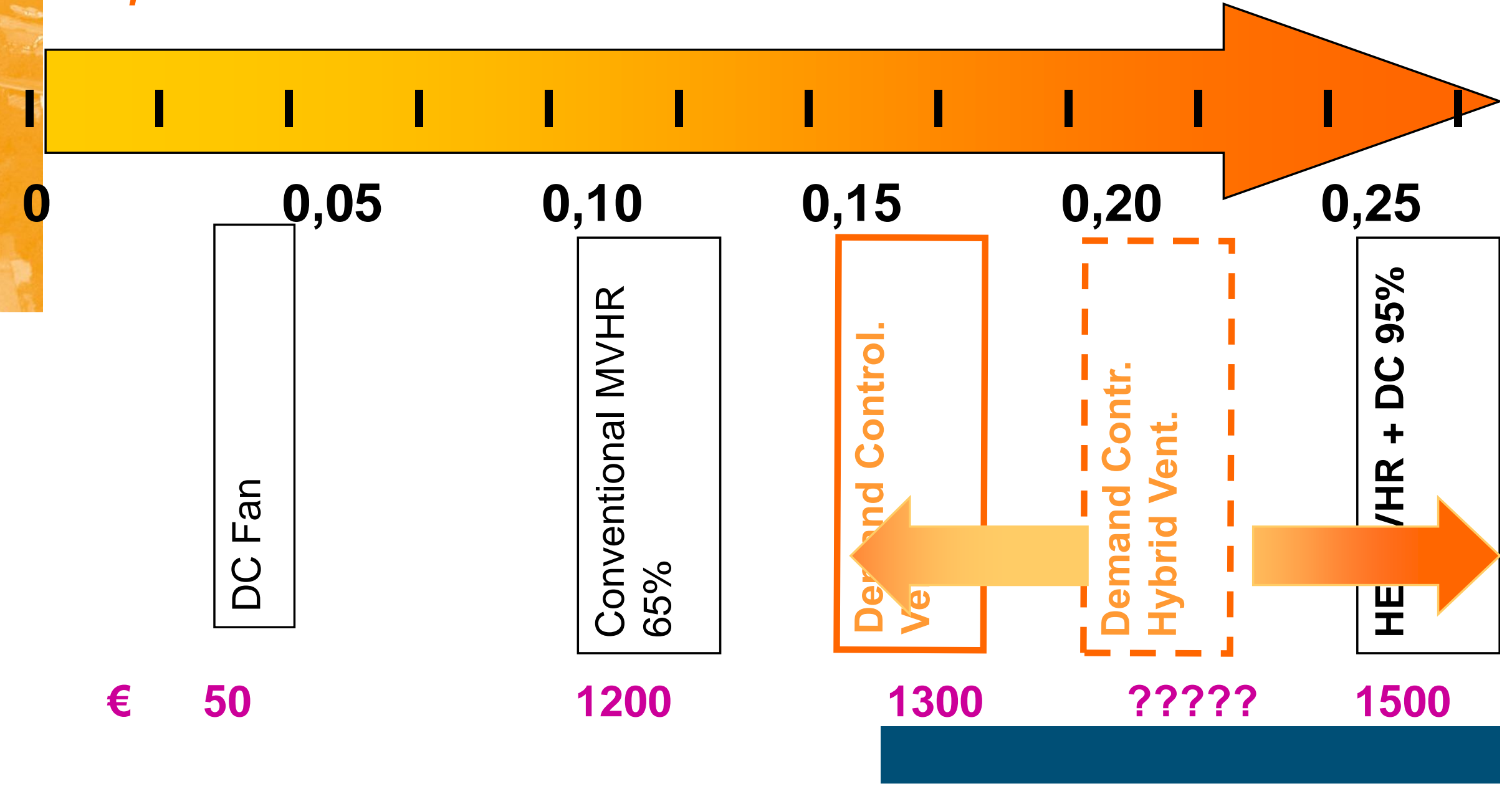




\section{Heat recovery units (counter flow)}

- EPBD default:

previously $75 \%$, currently $80 \%$

- EPBD certificates: $95 \%$

- Passive House certificates: 
Heat recovery units, lab. measurements by $\mathrm{PHI}$

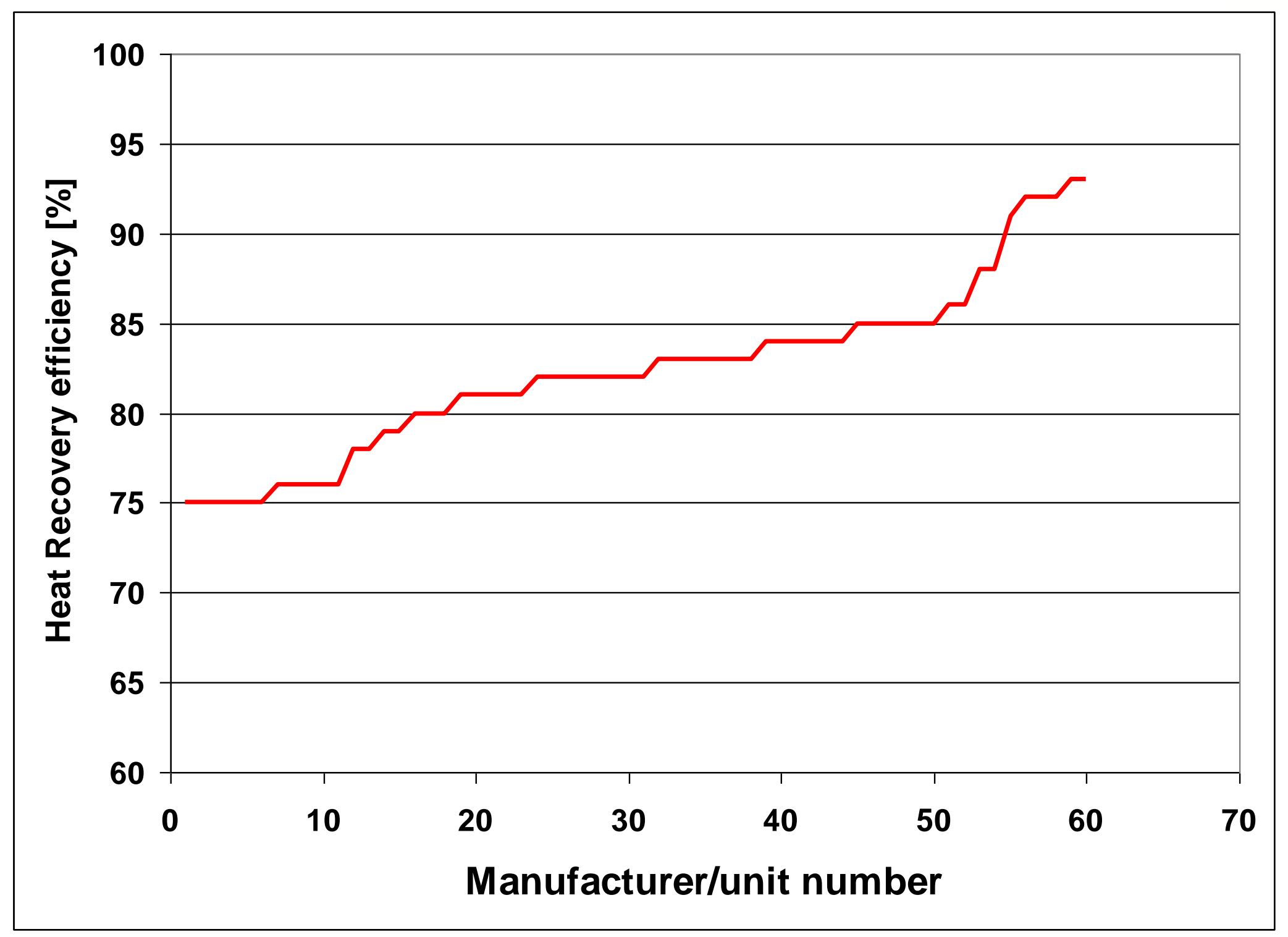


Heat recovery units, field measurements

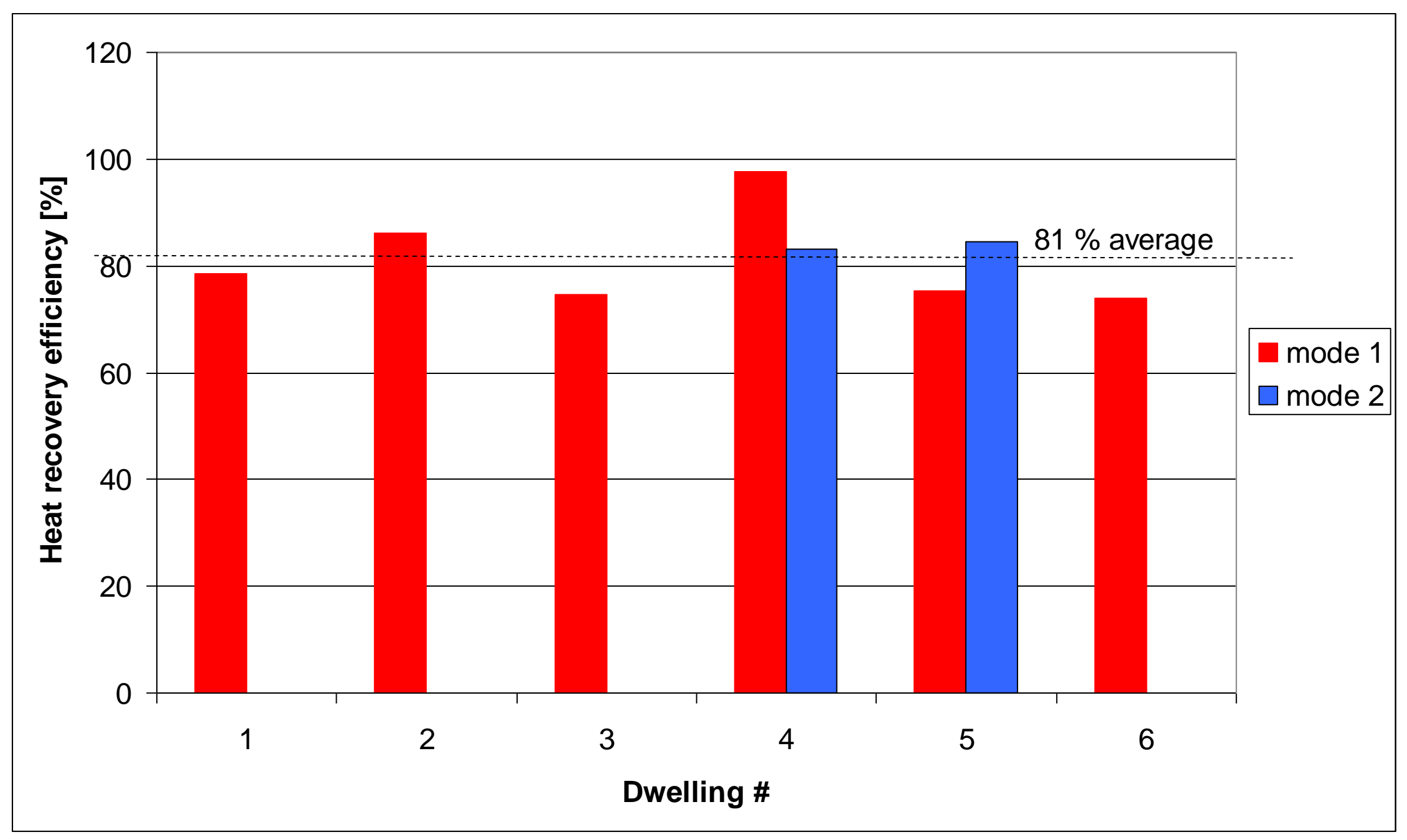




\section{DHW heat recovery units}
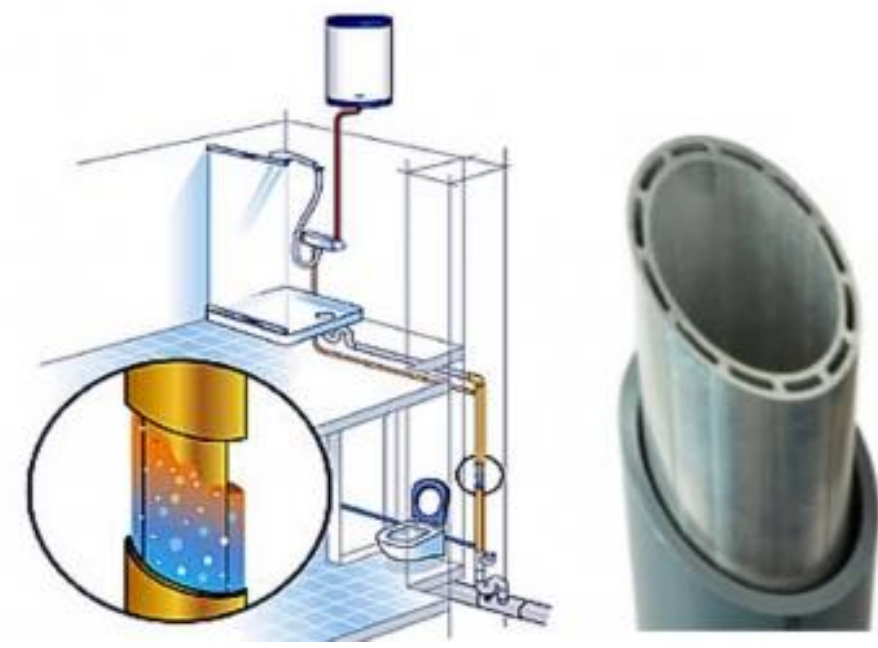

\section{Pattern A}
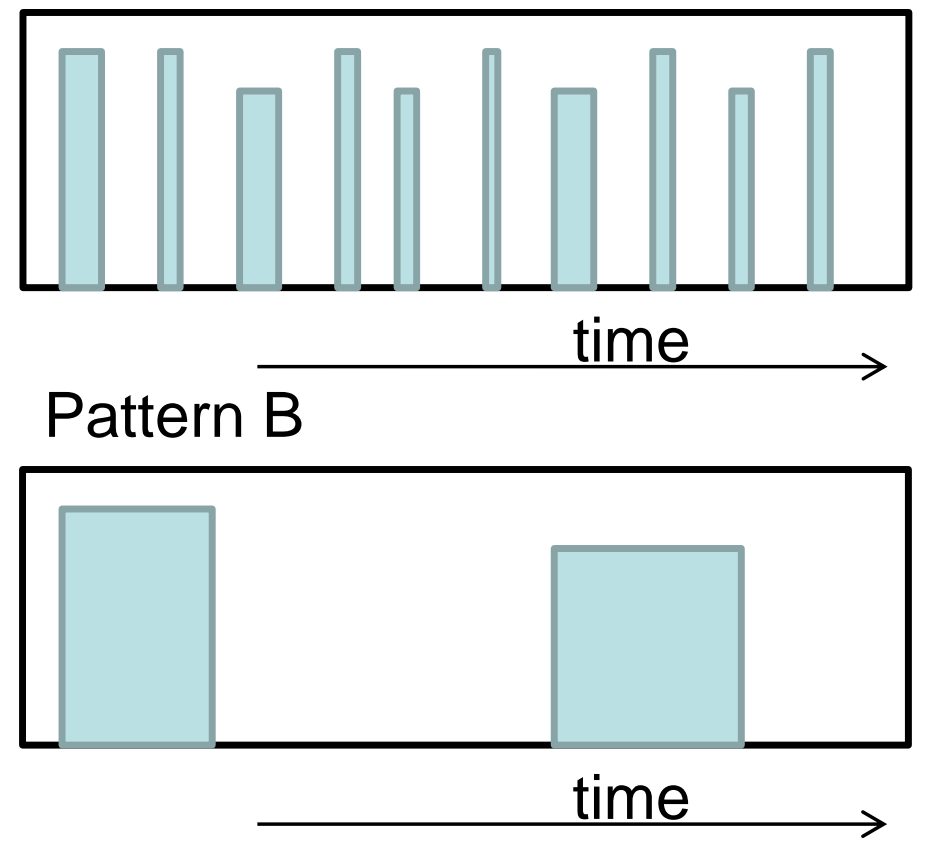

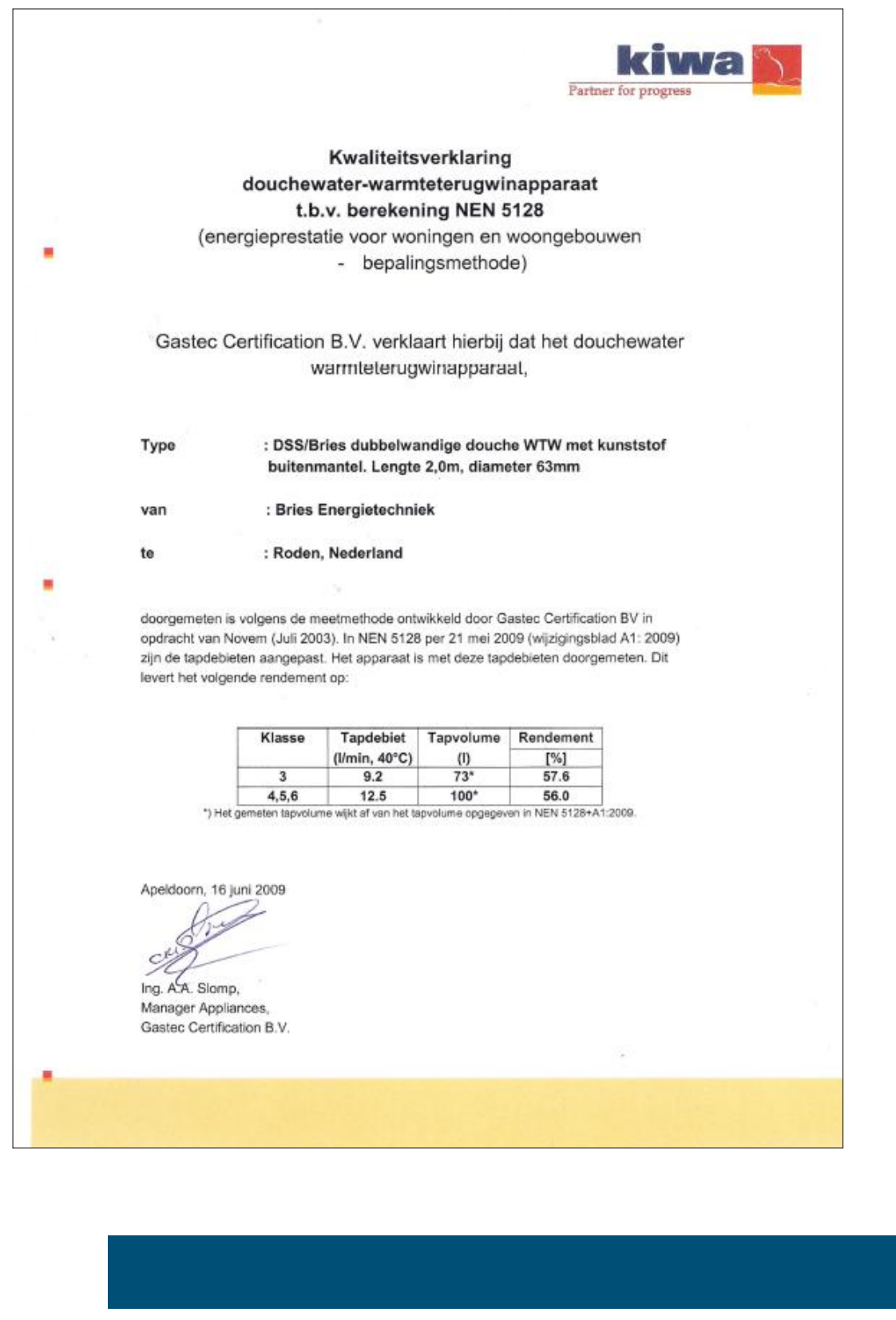




\section{What is it all about?}

\section{Redenen genoeg...}

....om te kiezen voor de Intergas Kombi Kompakt HRE

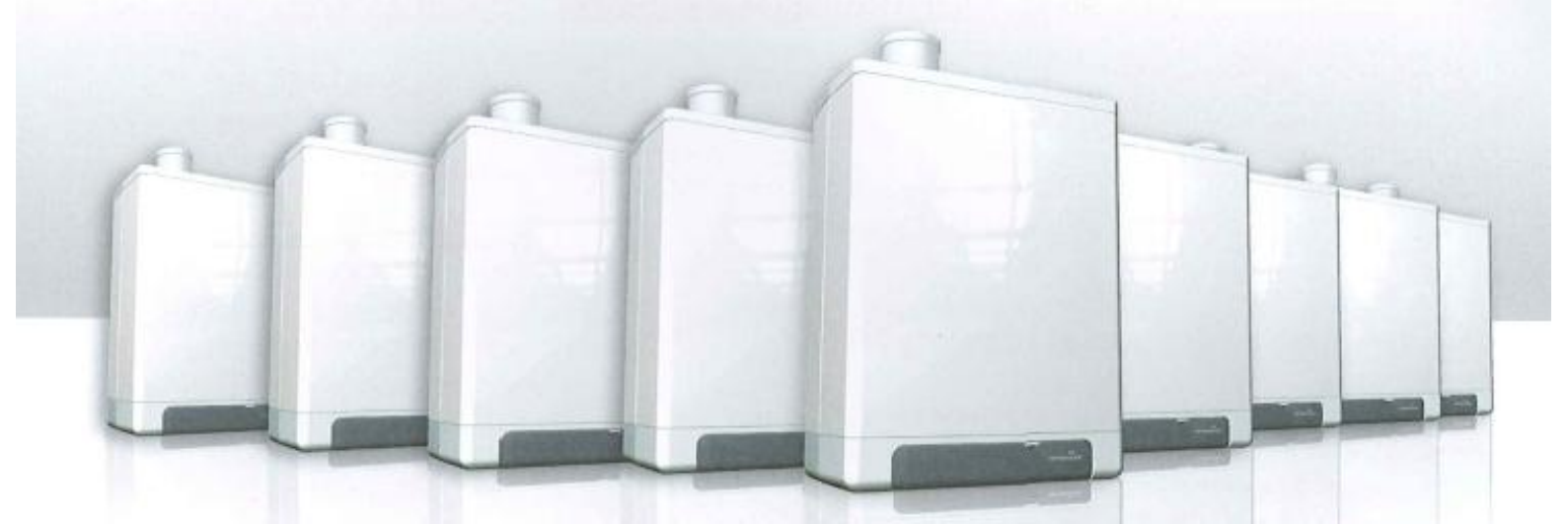

1 EPC verlaging met 0.19

2 Jaartaprendement tot $\mathbf{9 5 , 8 \%}$

3 Eenvoud in installatie en montage

4 Simpel te combineren

5 Maximaal rendement, tot maar liefst $42,5 \%$ daling $\mathrm{CO}_{2}$-emissie

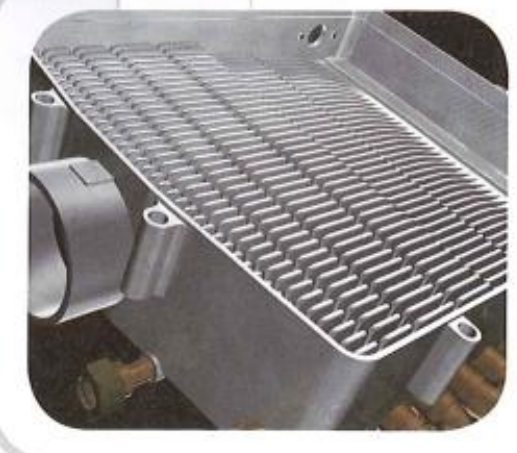




\section{Field data (I):small heatpumps}

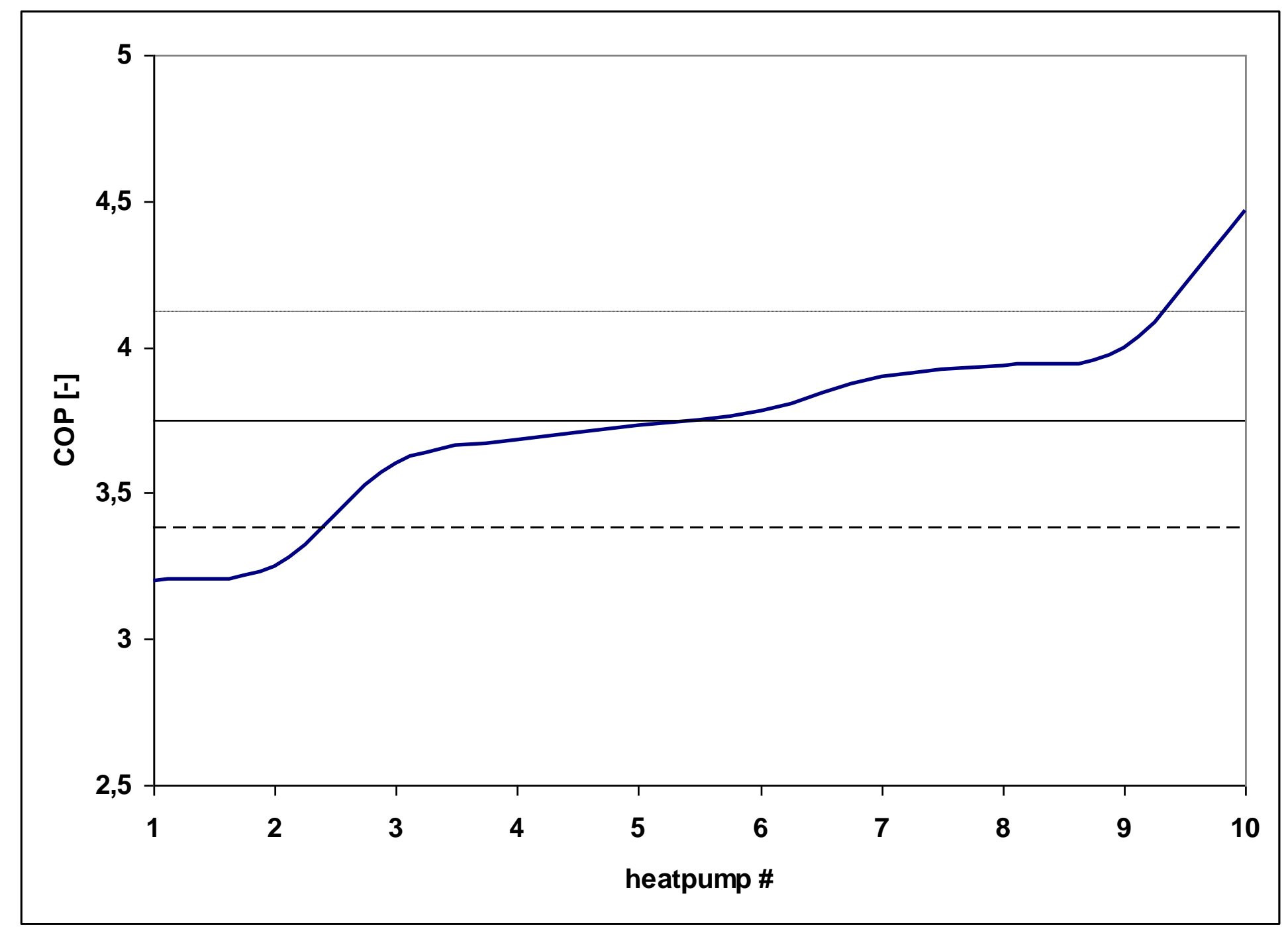




\section{COP of heatpumps}

\begin{tabular}{|l|l|l|l|}
\hline & $\begin{array}{l}\text { COP } \\
(\text { EPBD })\end{array}$ & $\begin{array}{l}\text { COP } \\
(\text { EPBD\& } \\
\text { certificate })\end{array}$ & $\begin{array}{l}\text { COP } \\
\text { (measured } \\
\text { average) }\end{array}$ \\
\hline $\begin{array}{l}\text { Space- } \\
\text { heating }\end{array}$ & 4,6 & 6,08 & \\
\hline DHW & $1,4 \times$ Corr. & $2,23 \times$ Corr. & \\
\hline Average & $4,1-4,3$ & $5,49-5,63$ & $3,75 \pm 0,5$ \\
\hline
\end{tabular}




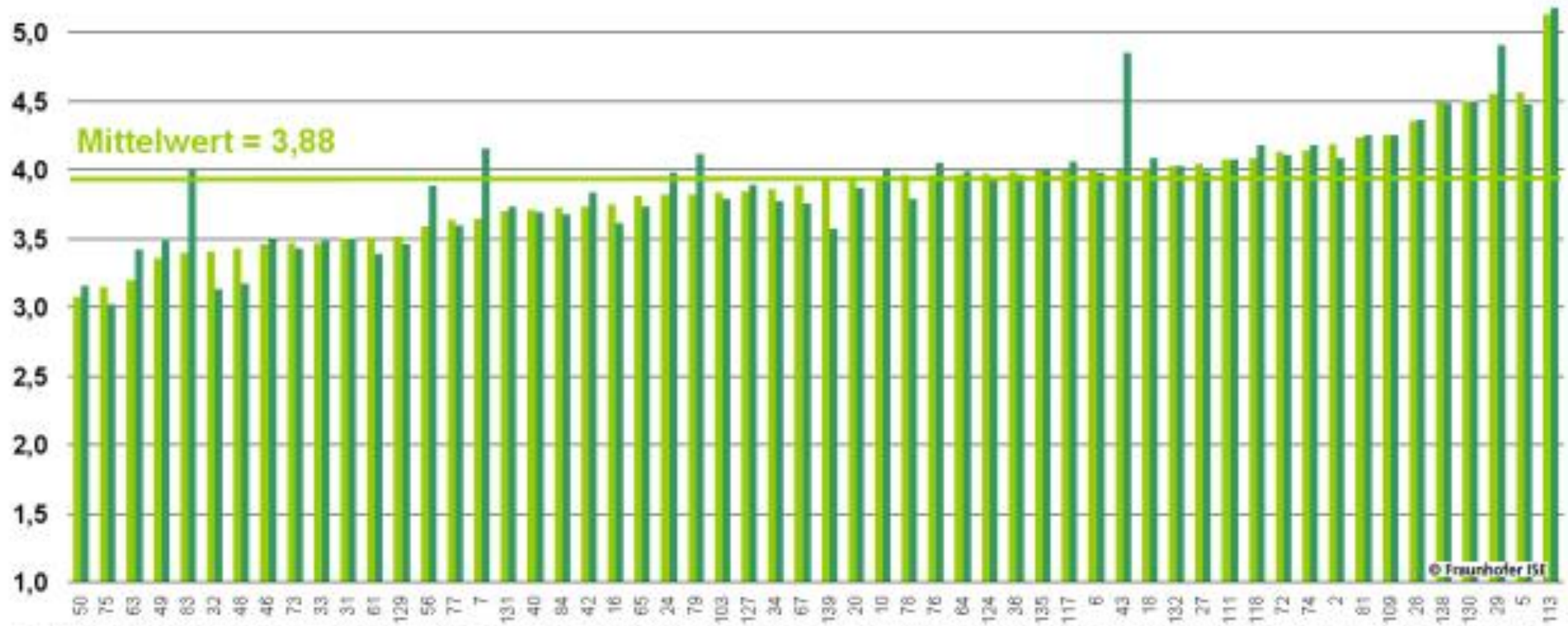

Bild 15: Arbeitszahlen von Erdreich-Wärmepumpen für die Zeiträume Juli 2007 bis Juni 2010 sowie Juli 2009 bis Juni 2010; die Anlagenbezeichnungen entsprechen den Herstellem bekannten ID-Nummern

Measured field data bij Fraunhofer (Ge) 


\section{Field data (II): large heatpumps}

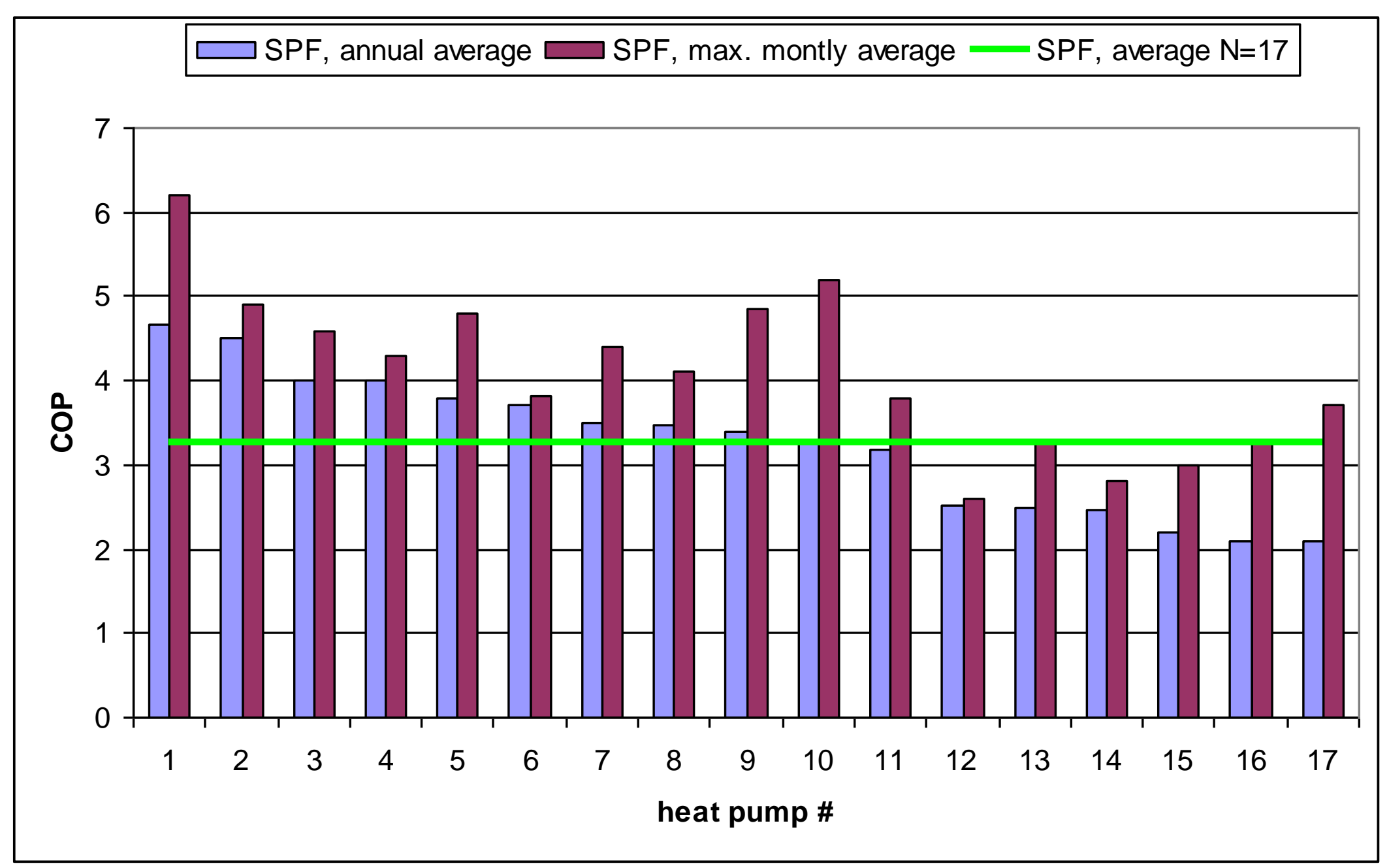




\section{Field data large heatpumps (II)}

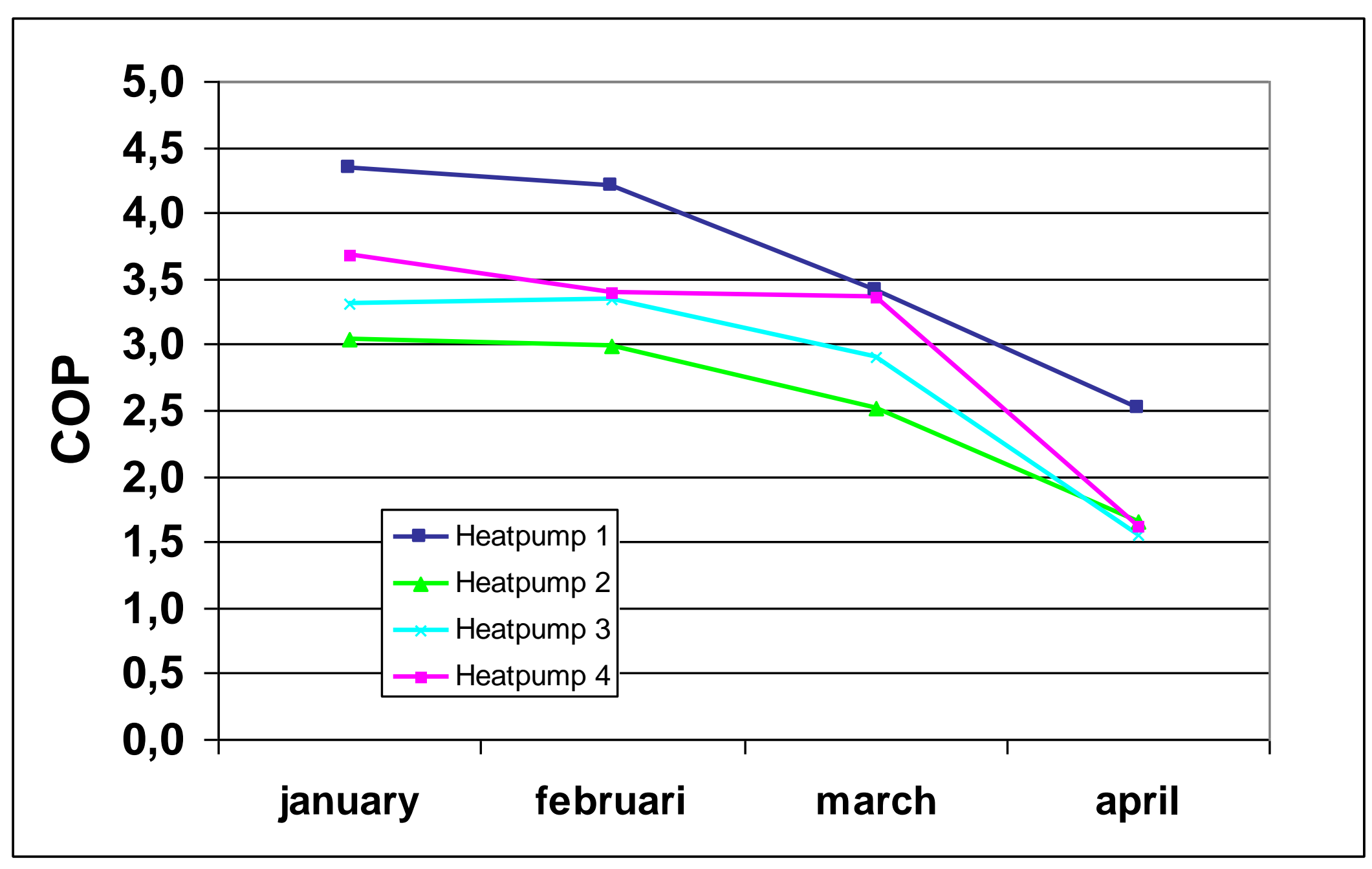




\section{Figure 23 Monthly heat efficiency (\%) against heat supplied - combination boilers}

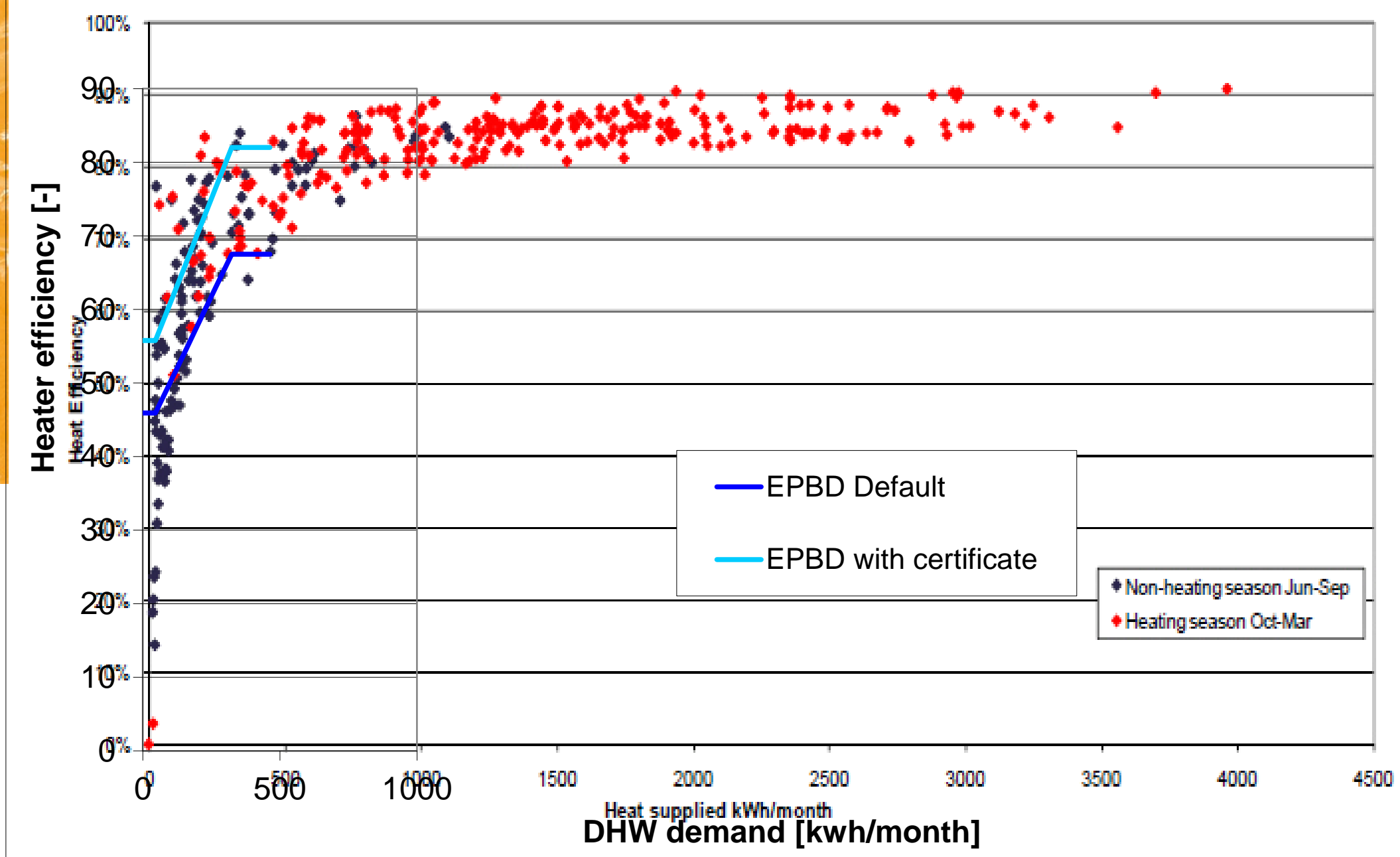

Field data on condensing gas-fired heaters bij Gastec at CRE, 2009 


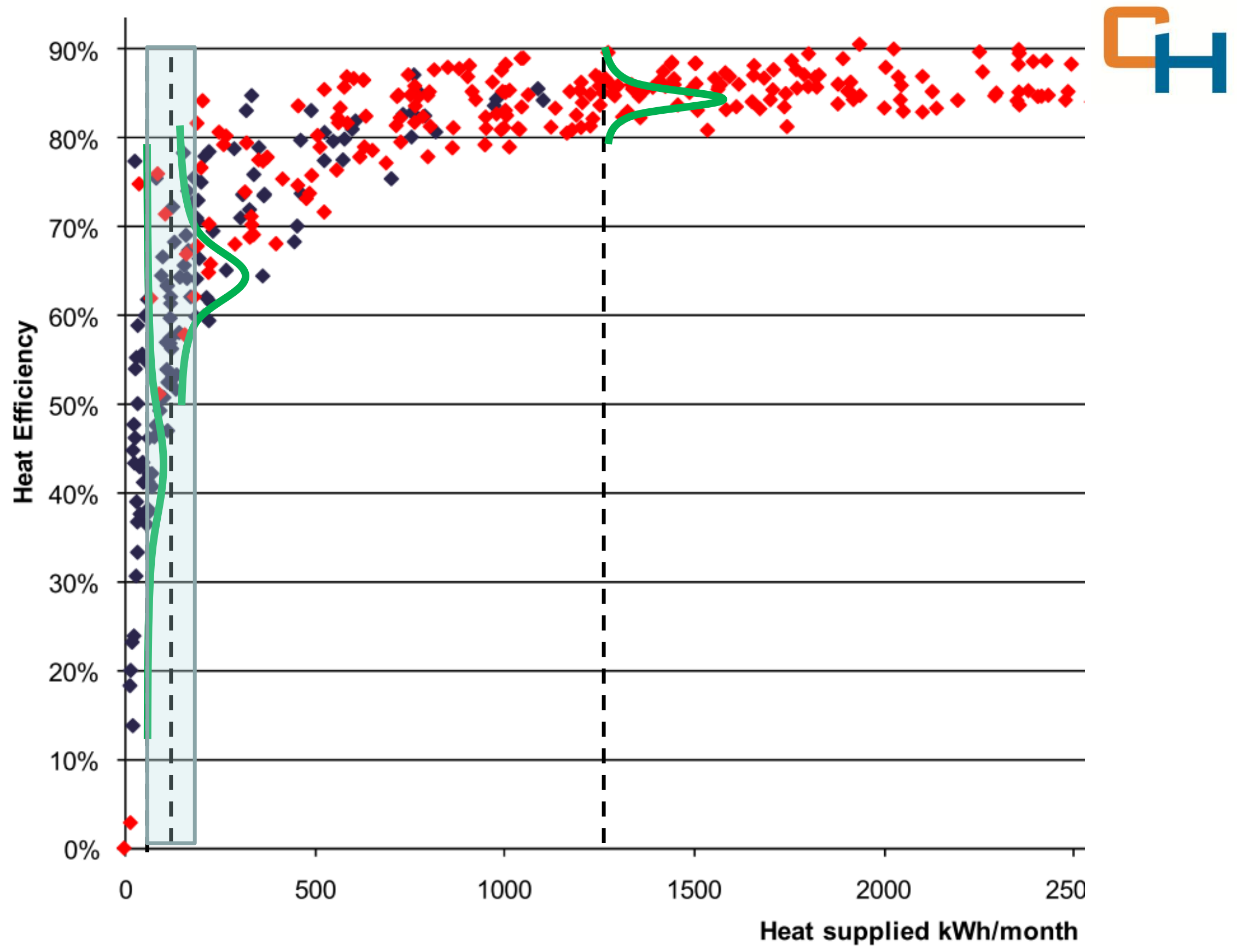




\section{Performance of combination of technologies??}

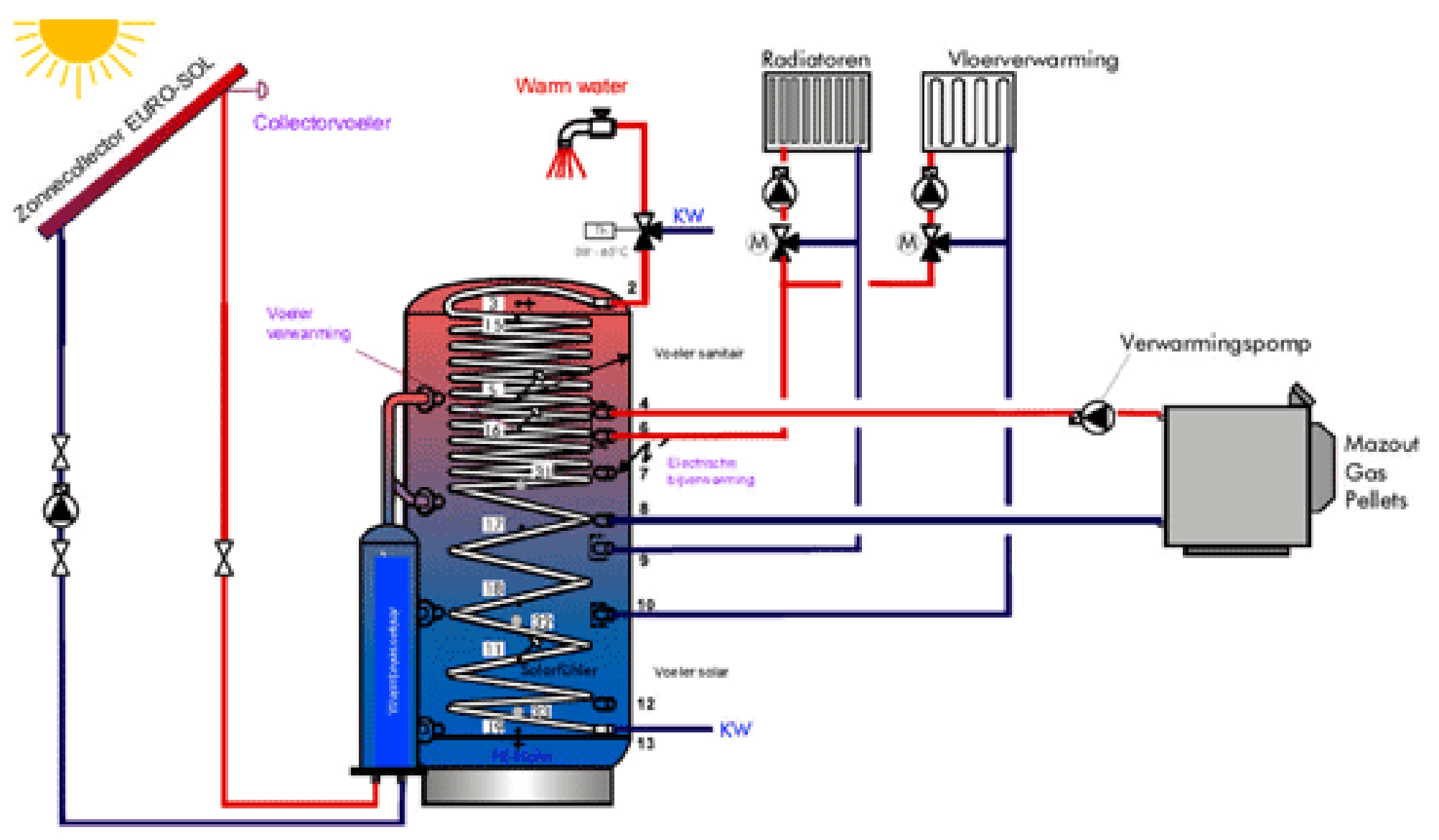


4. The occupant behaviour

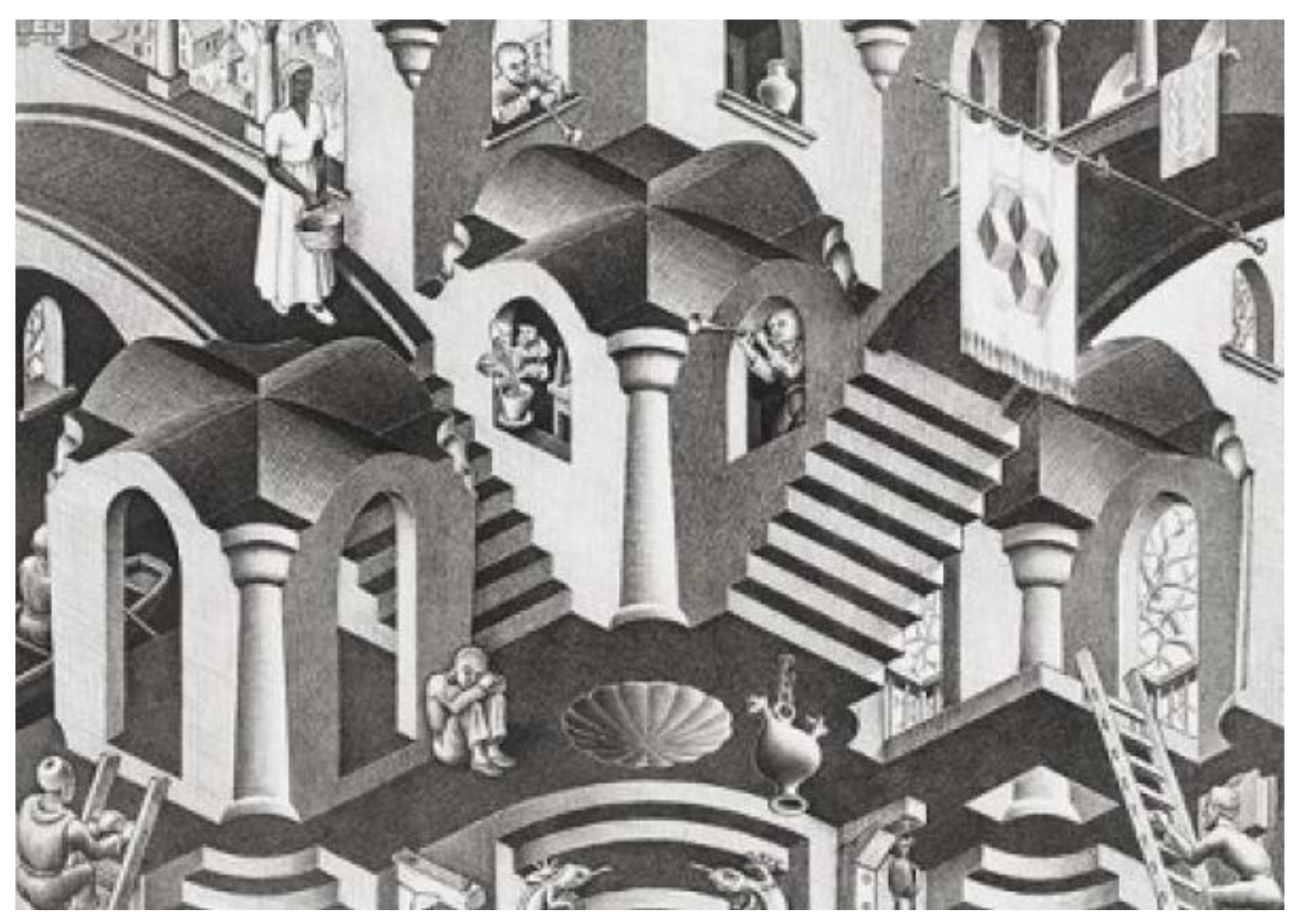




\section{The 4 stages of an occupant}

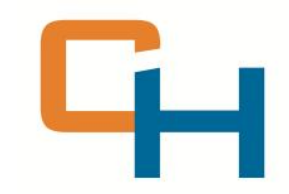

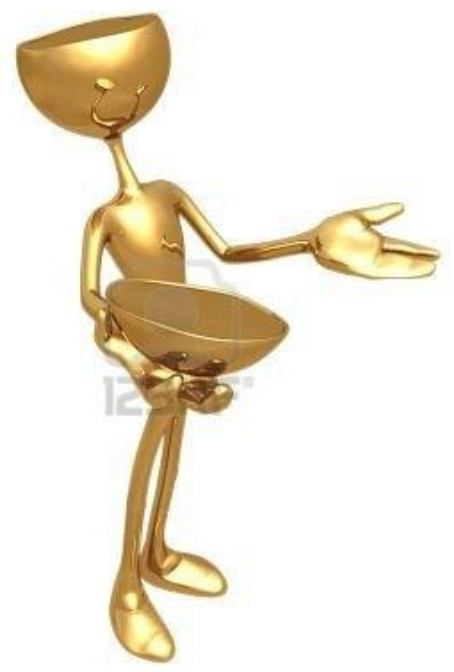

1. Easy stage

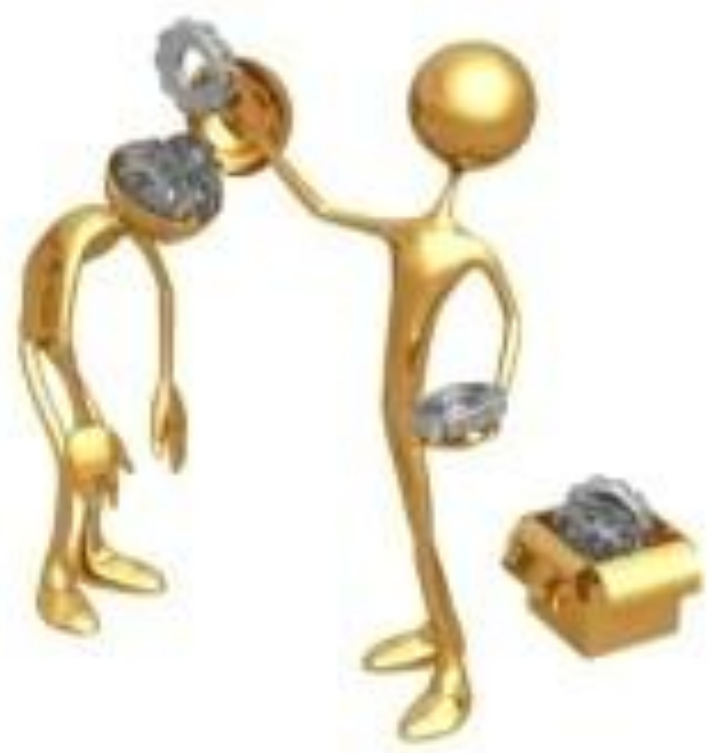

2. Thermal simulation stage

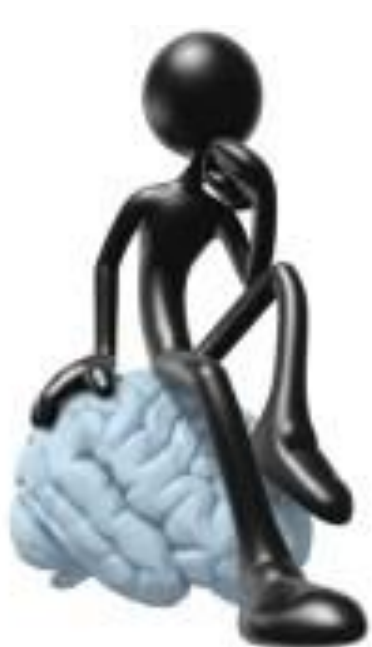

4. Real occupant

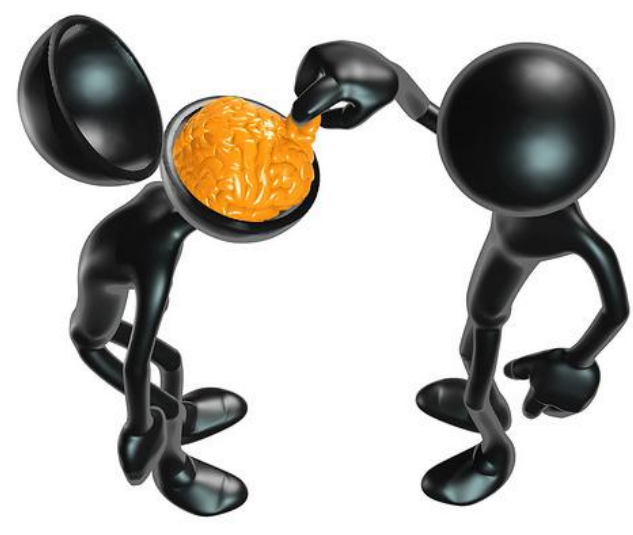

3. Comfort sensing stage 


\section{Energy use and occupant behaviour}

User aspects on energy use has been researched

- Social sciences

- Physics

- Economical sciences

From the social sciences several models have been developed

Exemples: Van Raaij model, NOA model 


\section{Behavioral model Van Raaij et al.}

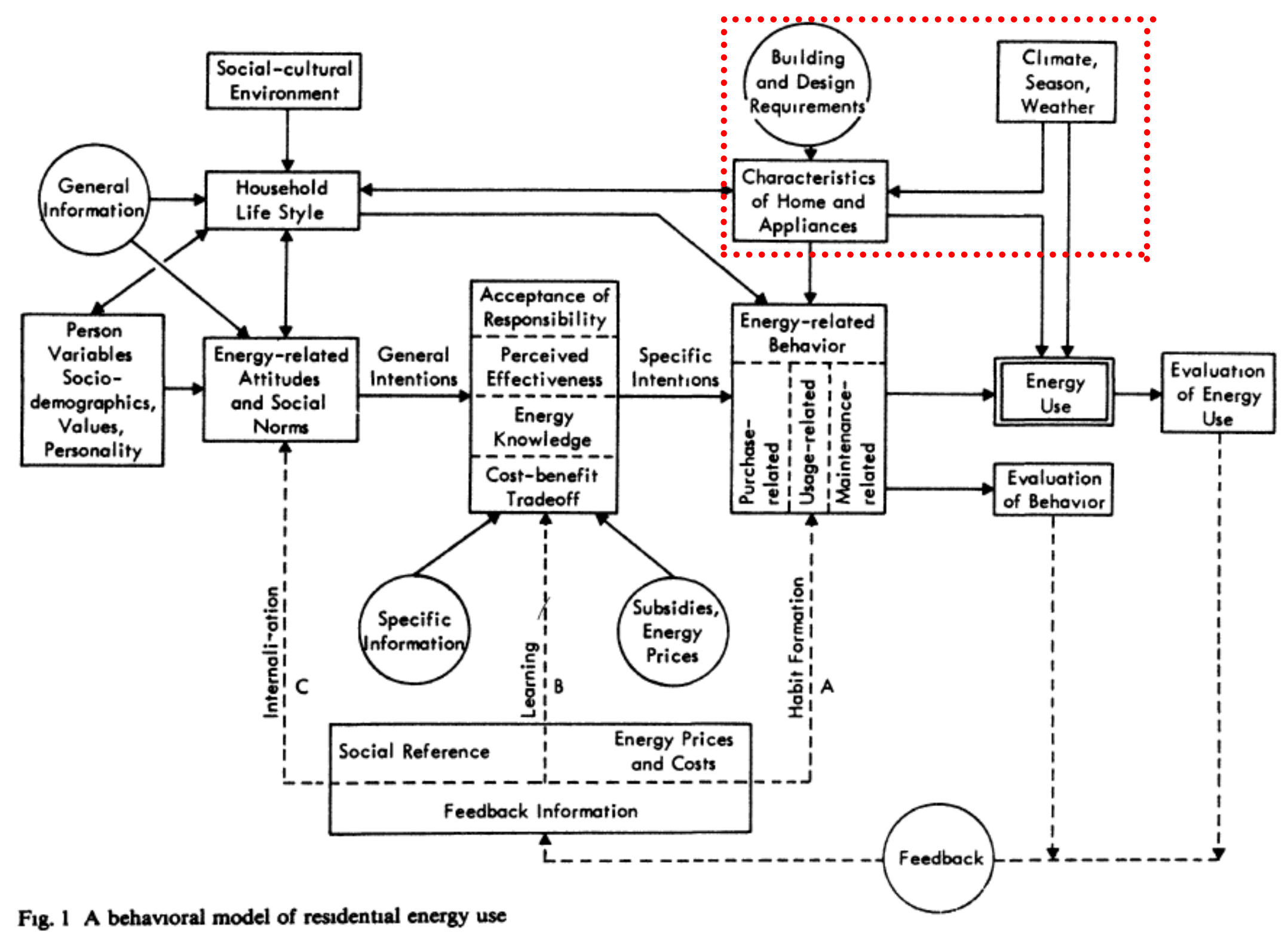




\section{NOA-model Gatersleben \& Vlek}

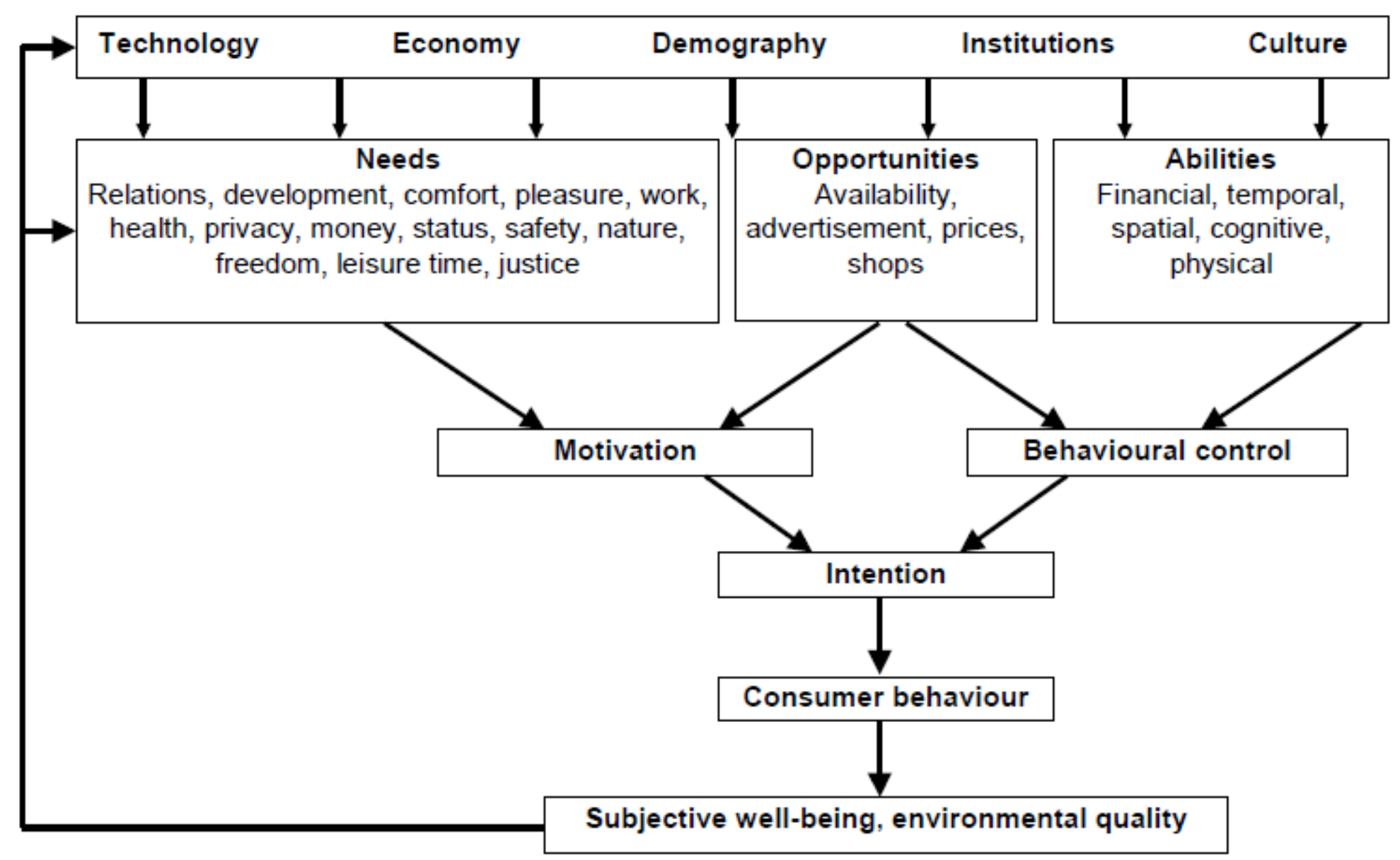




\section{Energy behaviour in dwellings}

\section{Tabel 2-17 Verklarende variabelen (naar afnemend effect) voor energiekosten}

\section{Energiekosten totaal}

\section{Gebruiksoppervlak}

Energie-index ${ }^{7}$

Wijze van verwarmen

Inkomen

Apparaatbezit en gebruik

Woningtype

Type huishouden

Stookpatroon

Aantal buitenzijden van de woning Leeftijd (hoofd huishouden)

Bouwjaar woning

Etnische achtergrond respondent

Wijze en mate van baden / douchen

Wijze en mate van ventileren

\section{Kosten elektriciteit}

Gebruiksoppervlak

Apparaatbezit en gebruik

Inkomen

Aantal kinderen

woningtype

Type huishouden

Wijze van verwarmen

stookpatroon

Aantal volwassenen

Etnische achtergrond respondent

Energie-index

$33 \%$ verklaarde variantie

\section{Kosten gas en warmte}

Wijze van verwarmen

Gebruiksoppervlak

Energie-index

stookpatroon

woningtype

Type huishouden

Wijze en mate van ventileren

Bouwjaar woning

Etnische achtergrond respondent

Inkomen

Aantal buitenzijden van de woning

Leeftijd (hoofd huishouden)

Aantal kinderen

$40 \%$ verklaarde variantie 


\section{Energy occupant behaviour}

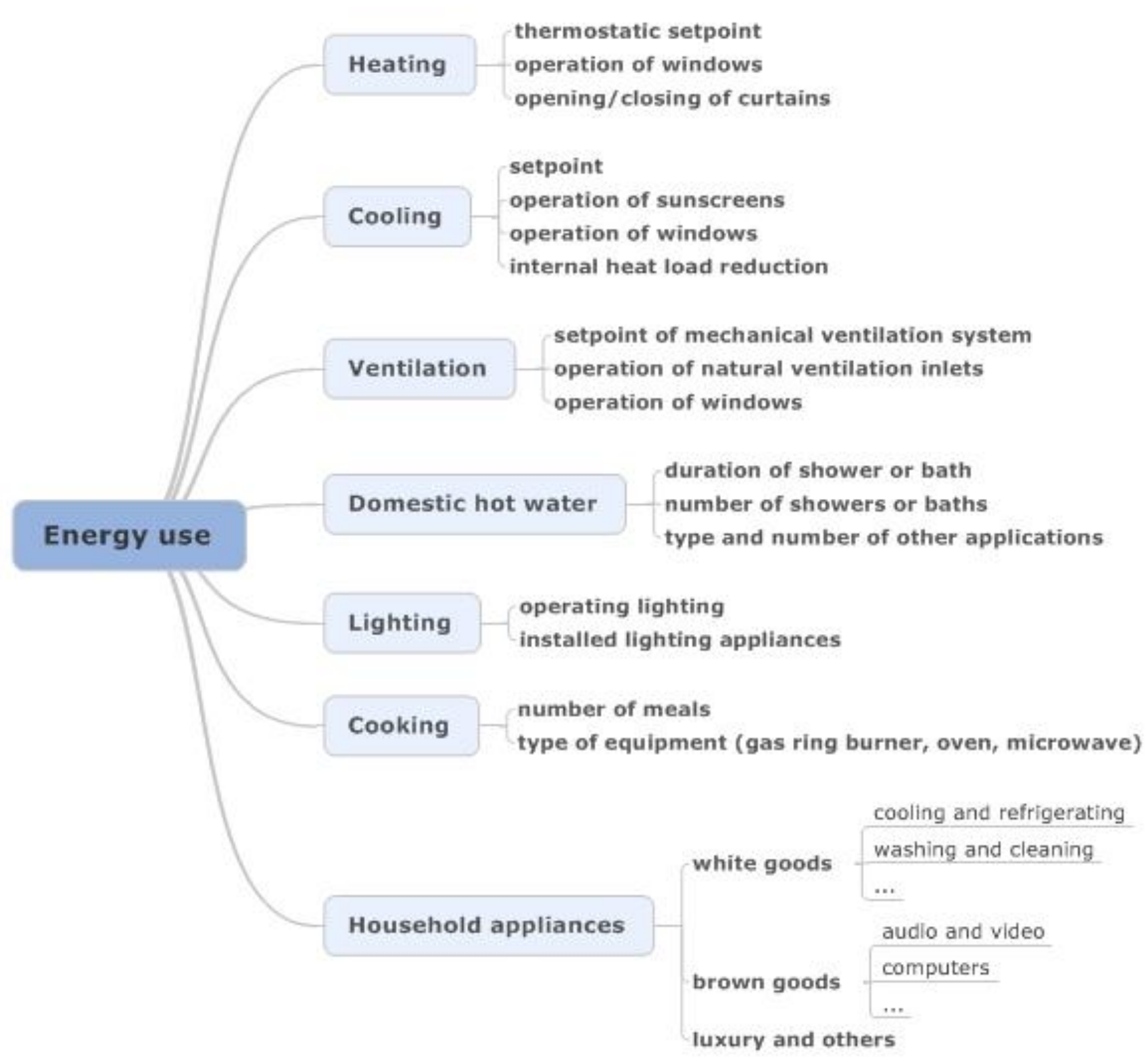




\section{Psychological aspects}

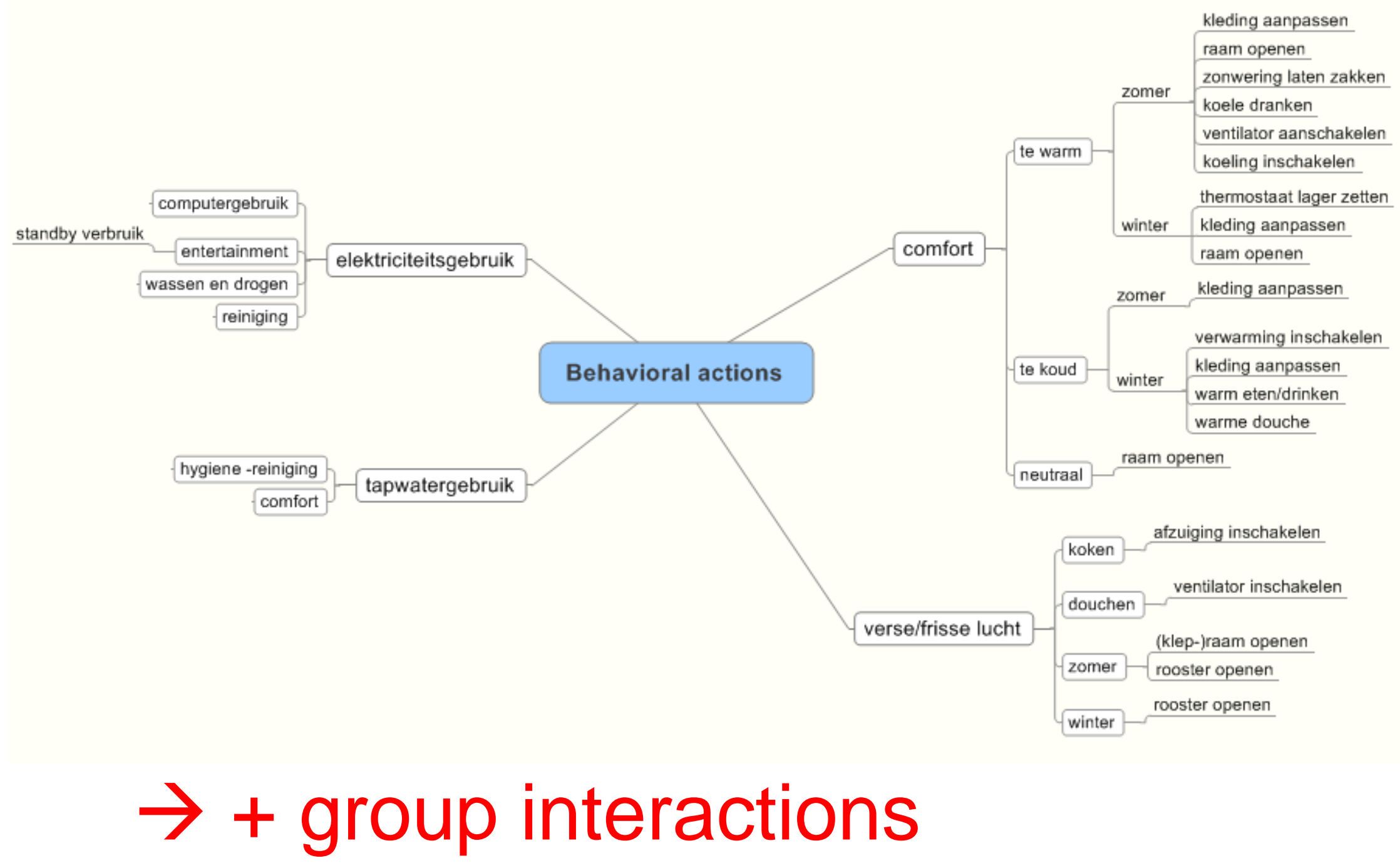




\section{The technical approach}

Thermostatic setpoint
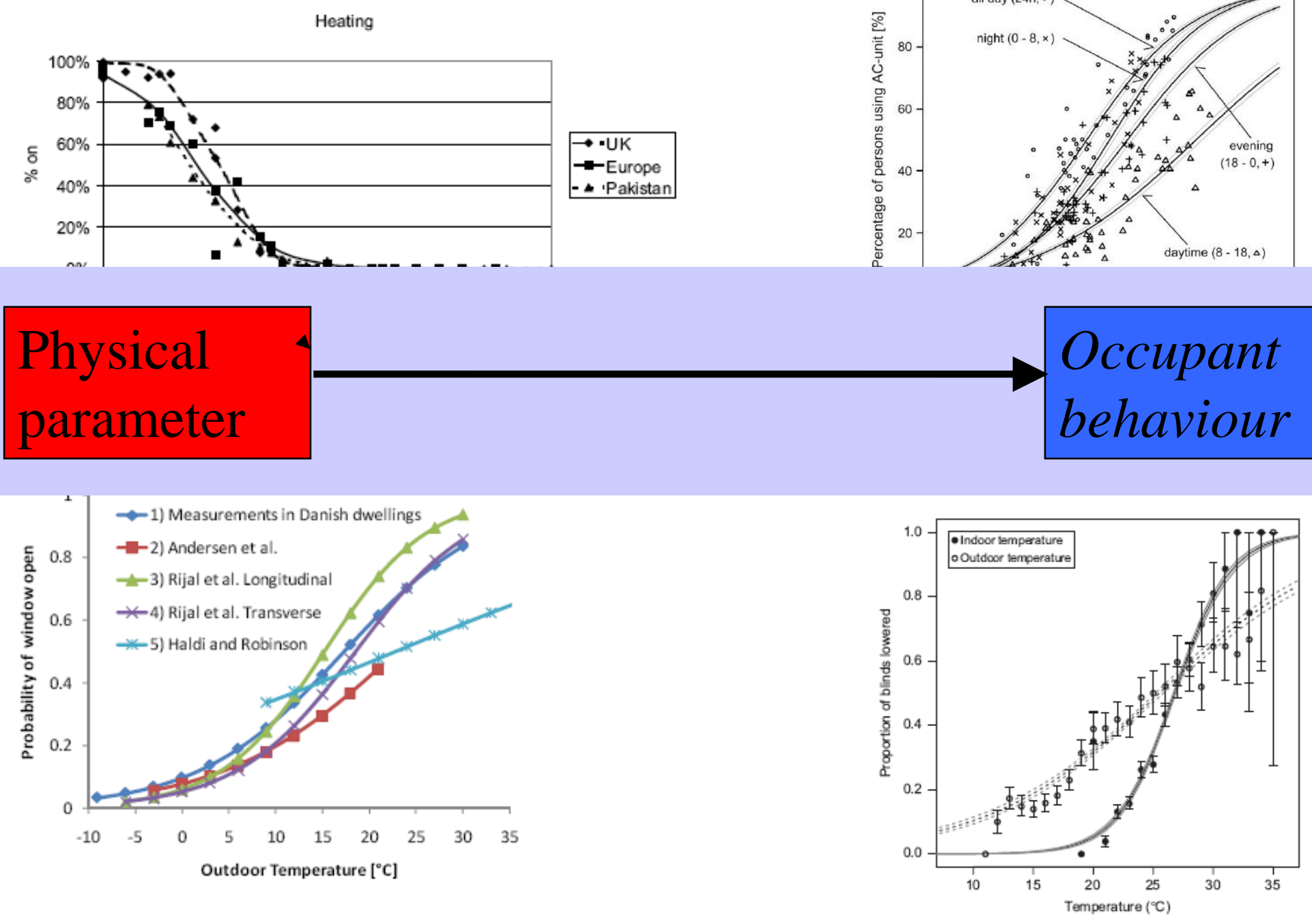

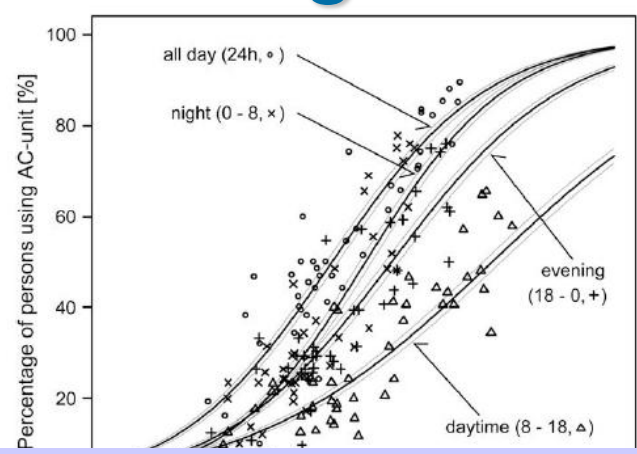

Occupant behaviour 


\section{What is the complex reality?}

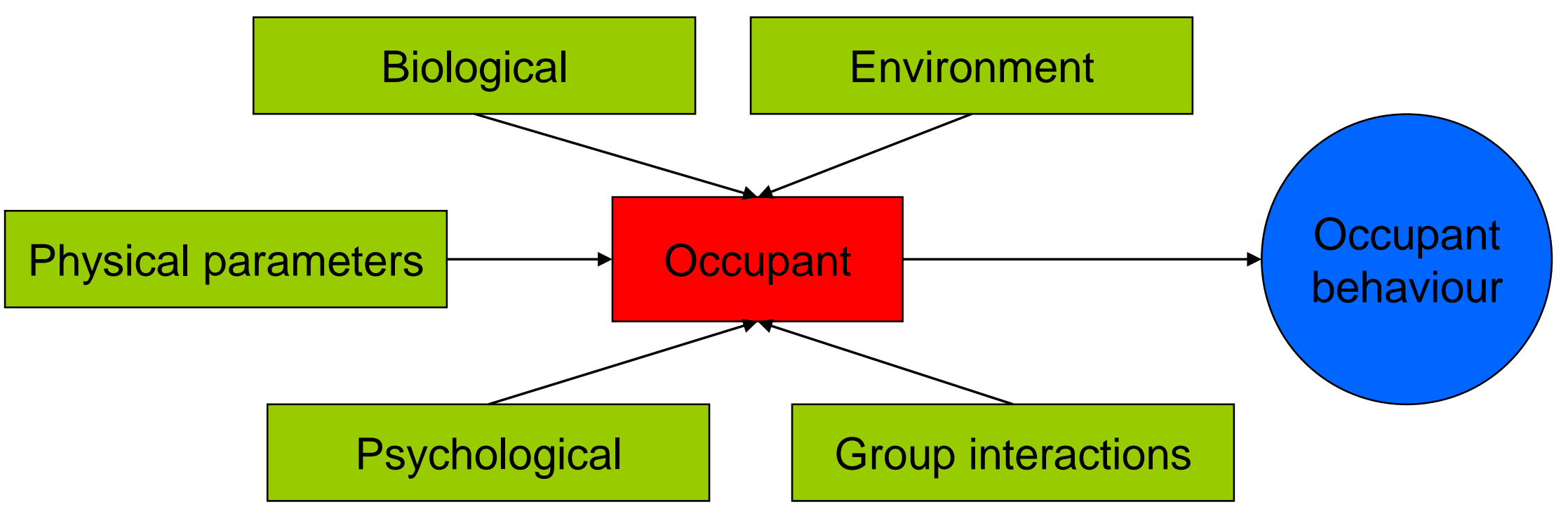




\section{Occupant behaviour and energy}

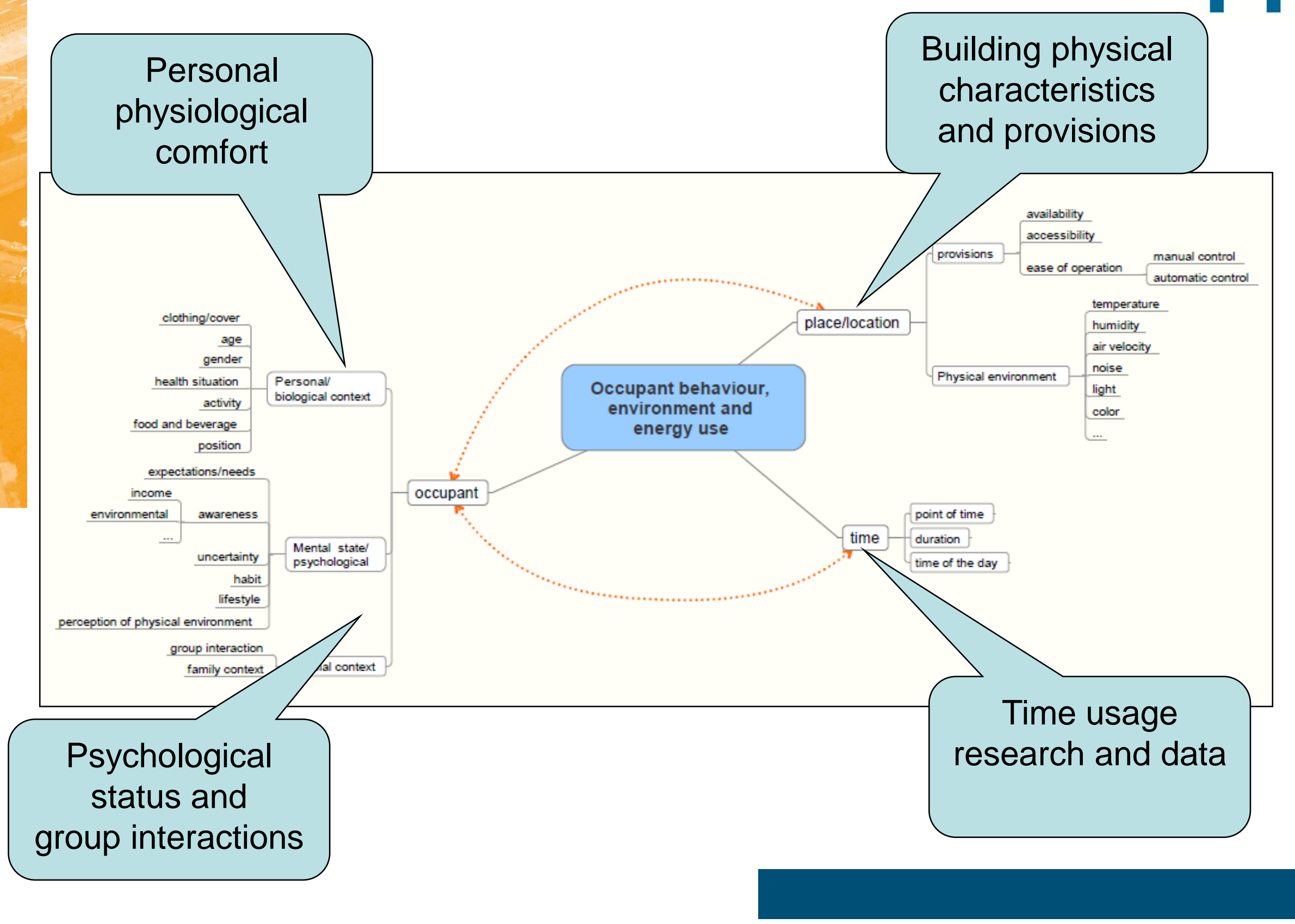




\section{Occupancy model}

What type of calculation model is appropriate?

- Stochastic model, e.g. Markov-Chain Monte Carlo

Deterministic model, e.g. occupancy profile

- Semi probabilistic/deterministic model 


\section{Occupancy}

What data is available?

- Time-use survey from "The Netherlands Institute for Social Research"

- Database with 2000 respondents

- Time-interval: 15 minutes, duration: 1 week

- Activities of people have been translated in absence or presence (time and location) 


\section{Time-use survey}

区 Microsoft Excel - Kopie van Kopie van 1_p_henk.sls

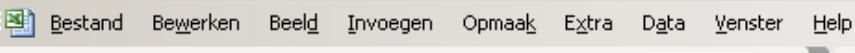

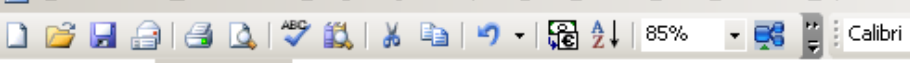
$-\underline{-a} x$

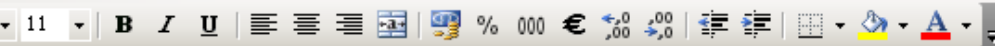

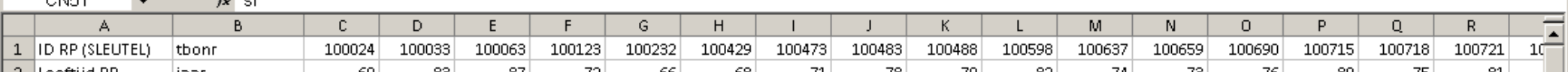

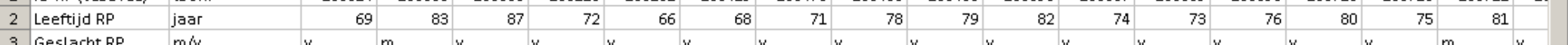

3 Geslacht RP $\mathrm{m} / \mathrm{N}$

4 Plaats huishoude $h k / p h k / k / 0$

21 Su. 00

\begin{tabular}{lll}
21 & Su. 00 \\
22 & Su. 00.15 \\
\hline 23 & Su.00.30
\end{tabular}

23 Su. 00.30

\begin{tabular}{l|l|l|}
24 & Su. 00.45 \\
\hline 25 & Su. 01 \\
\hline
\end{tabular}

26 Su. 01.15

27 Su. 01.30

28 Su. 01.45

\begin{tabular}{|l|l|}
29 & Su.02 \\
\hline
\end{tabular}

\begin{tabular}{l|l|}
30 & Su. 02.15 \\
\hline 31 & Su. 02.30
\end{tabular}

\begin{tabular}{ll}
31 & Su. 02.30 \\
\hline 32 & Su 02.45 \\
\hline
\end{tabular}

33 Su. 03

\begin{tabular}{|l|l|}
\hline 34 & Su. 03.15 \\
\hline 35 & Su 03.30 \\
\hline
\end{tabular}

\begin{tabular}{|l|l|}
35 & Su. 03.30 \\
\hline 36 & Su. 03.45 \\
\hline
\end{tabular}

37 Su.04

38 Su. 04.15

\begin{tabular}{|l|l|}
\hline 39 & Su. 04.30 \\
\hline 40 & Su. 04.45 \\
\hline
\end{tabular}

41 Su. 05

\begin{tabular}{|l|l|}
\hline 42 & Su. 05.15 \\
\hline 43 & Su 05.30 \\
\hline
\end{tabular}

\begin{tabular}{ll}
43 & Su. 05.30 \\
\hline 44 & Su 05.45
\end{tabular}

45 Su.06

\begin{tabular}{|l|l|}
\hline 45 & Su. 06 \\
\hline 46 & Su. 06.15 \\
\hline 47 & Su.
\end{tabular}

\begin{tabular}{|l|l|}
\hline 46 & Su. 06.15 \\
\hline 47 & Su. 06.30 \\
\hline
\end{tabular}

48 Su. 06.45

49 Su. 07

\begin{tabular}{|l|l|}
\hline 50 & Su. 07.15 \\
\hline 51 & Su. 07.30 \\
\hline
\end{tabular}

52 Su. 07.45

53 Su. 08

54 Su. 08.15

55 Su.08.30

\begin{tabular}{|l|l|}
\hline 56 & Su. 08.45 \\
\hline 57 & Su. 09 \\
\hline
\end{tabular}

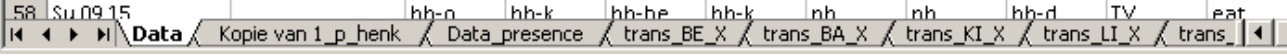

\begin{tabular}{|c|c|c|c|c|c|c|c|c|c|c|c|c|c|c|c|c|}
\hline hk & hk & hk & hk & hk & hk & hk & hk & hk & hk & hk & hk & hk & hk & hk & hk & hk \\
\hline sl & sl & sl & sl & sl & sl & sl & sl & sl & sl & sl & sl & sl & sl & sl & sl & sl \\
\hline sl & sl & sl & sl & sl & sl & sl & sl & sl & sl & sl & sl & sl & sl & sl & sl & sl \\
\hline sl & sl & sl & sl & sl & sl & sl & sl & sl & sl & sl & sl & sl & sl & sl & sl & sl \\
\hline sl & sا & sl & sl & sl & sl & sl & sl & sl & sl & sl & sl & sl & sl & sl & sl & sl \\
\hline sl & sl & sl & sl & sl & sl & sl & sl & sl & sl & sl & sl & sl & sl & sl & sl & sl \\
\hline sl & sl & sl & sl & sl & sl & sl & sl & sl & sl & sl & sl & sl & sl & sl & sl & sl \\
\hline sl & sا & sl & sl & sl & sl & sا & sl & sl & sl & sl & sl & sl & sl & sl & sl & sl \\
\hline sl & sl & sl & sl & sl & sl & sl & sl & sl & sl & sl & sl & sl & sl & sl & sl & sl \\
\hline sl & sl & sl & sl & sl & sl & sl & sl & sl & sl & sl & sl & sl & sl & sl & sl & sl \\
\hline sl & sl & sl & sl & sl & sl & sl & sl & sl & sl & sl & sl & sl & sl & sl & sl & sl \\
\hline sl & sl & sl & sl & sl & sl & sl & sl & sl & sl & sl & sl & sl & sl & sl & sl & sl \\
\hline sl & sl & sl & sl & sl & sl & sl & sl & sl & sl & sl & sl & sl & sl & sl & sl & sl \\
\hline sl & sl & sl & sl & sl & sl & sا & sl & $s \mid$ & sl & sl & sl & sl & sl & sl & sl & sl \\
\hline sl & sl & sl & sl & sl & sl & sl & sl & sl & sl & sl & sl & sl & sl & sl & sl & sl \\
\hline sl & sl & sl & sl & sl & sl & sl & sl & sl & sl & sl & sl & sl & sl & sl & sl & sl \\
\hline sl & sl & sl & sl & sl & sl & sl & sl & sl & sl & sl & sl & sl & sl & sl & sl & sl \\
\hline sl & sl & sl & sl & sl & sl & sl & sl & sl & sl & sl & sl & sl & sl & sl & sl & sl \\
\hline sl & sl & sl & sl & sl & sl & sl & sl & sl & sl & sl & sl & sl & sl & sl & sl & sl \\
\hline sl & sl & sl & sl & sl & sl & sl & sl & sl & sl & sl & sl & sl & sl & sl & sl & sl \\
\hline sl & sl & sl & sl & sl & sl & sl & sl & sl & sl & sl & sl & sl & sl & sl & sl & sl \\
\hline sl & sl & sl & sl & sl & sl & sl & sl & sl & sl & sl & sl & sl & sl & sl & sl & sl \\
\hline sl & sl & sl & sl & sl & sl & sl & sl & sl & sl & sl & sl & sl & sl & sl & sl & sl \\
\hline sl & sl & sl & sl & sl & sl & sl & sl & sl & sl & sl & sl & sl & sl & sl & sl & sl \\
\hline sl & sl & sl & sا & sl & sl & sl & sl & sl & sl & sl & sl & sl & sl & sl & sl & sl \\
\hline sl & sl & sl & sl & sl & sl & sl & sl & sl & sl & sl & sl & sl & sl & sl & sl & sl \\
\hline sl & sl & sl & sl & sl & sl & sl & sl & sl & sl & sl & sl & sl & sl & sl & sl & sl \\
\hline sl & sl & sl & sl & sl & sl & sl & sl & sl & sl & sl & sl & sl & sl & sl & sl & sl \\
\hline sl & sl & sl & sا & sl & sl & sl & sl & sl & sl & sl & sl & sl & sl & sl & sl & sl \\
\hline sl & sl & sl & sl & $\mathrm{pc}$ & sl & sl & sl & sl & sl & sl & sl & sl & sl & sl & sl & sl \\
\hline sl & sl & sl & sl & $\mathrm{pc}$ & sl & sl & sl & sl & sl & sl & sl & sl & sl & sl & sl & sl \\
\hline sl & sl & sl & sl & pc & sl & sl & pC & pc & cook & sl & sl & cook & sl & sl & sl & sl \\
\hline sl & sl & $p c$ & hh-d & pC & sl & sl & pC & eat & cook & sl & sl & cook & sl & sl & sl & sl \\
\hline hh-o & $p c$ & $p c$ & cook & cook & pc & sl & eat & eat & $s 1-s$ & sl & eat & cook & $p c$ & sl & $\mathrm{pc}$ & pc \\
\hline hh-0 & cook & hh-d & cook & eat & pc & sl & eat & eat & pc & sl & eat & hh-k & $\mathrm{pc}$ & sl & $\mathrm{pc}$ & eat \\
\hline hh-o & cook & eat & hh-d & eat & $\mathrm{pC}$ & sl & TV & pc & hh-d & eat & eat & hh-k & ho & sl & eat & eat \\
\hline hh-0 & cook & eat & hh-k & $h h-k$ & $\mathrm{pc}$ & sl & TV & nh & hh-k & eat & eat & $p c$ & cook & sl & eat & eat \\
\hline hh-o & hh-d & hh-k & hh-k & nh & nh & cook & TV & $\mathrm{nh}$ & read & eat & pc & $p c$ & cook & $p c$ & read & hh-t \\
\hline & & & & & & & & & reard & pat & $\mathrm{nr}$ & $\mathrm{nh}$ & hh-r & $\mathrm{nr}$ & rear & $h_{-1}$ \\
\hline
\end{tabular}


Modelling occupancy and building

Sub-selection from the Time-use data used in building model:

- Two retired persons

- Two working persons

- Family: 2 parents and 2 children

Building model:

- Reference single-family dwelling

- TRNSYS simulation

- Heating in specific room on during occupancy 
Markov-Chain

Monte Carlo (MCMC)

Outline of MCMC procedure for occupancy modelling

- Define 6 states: bedroom, bathroom, kitchen, living room, other, not at home

- Derive transition probabilities from one state to

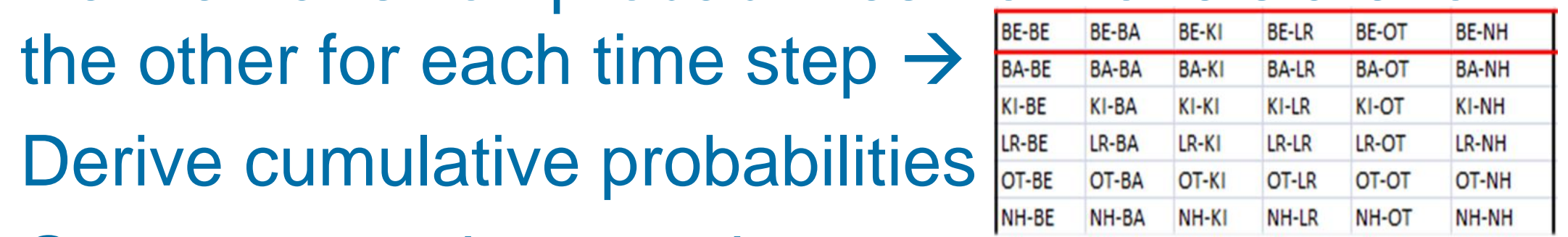

- Generate random numbers and use cumulative transition probabilities to find new state 


\section{Transition probability}

Example transition probability: bedroom $\rightarrow$ bedroom

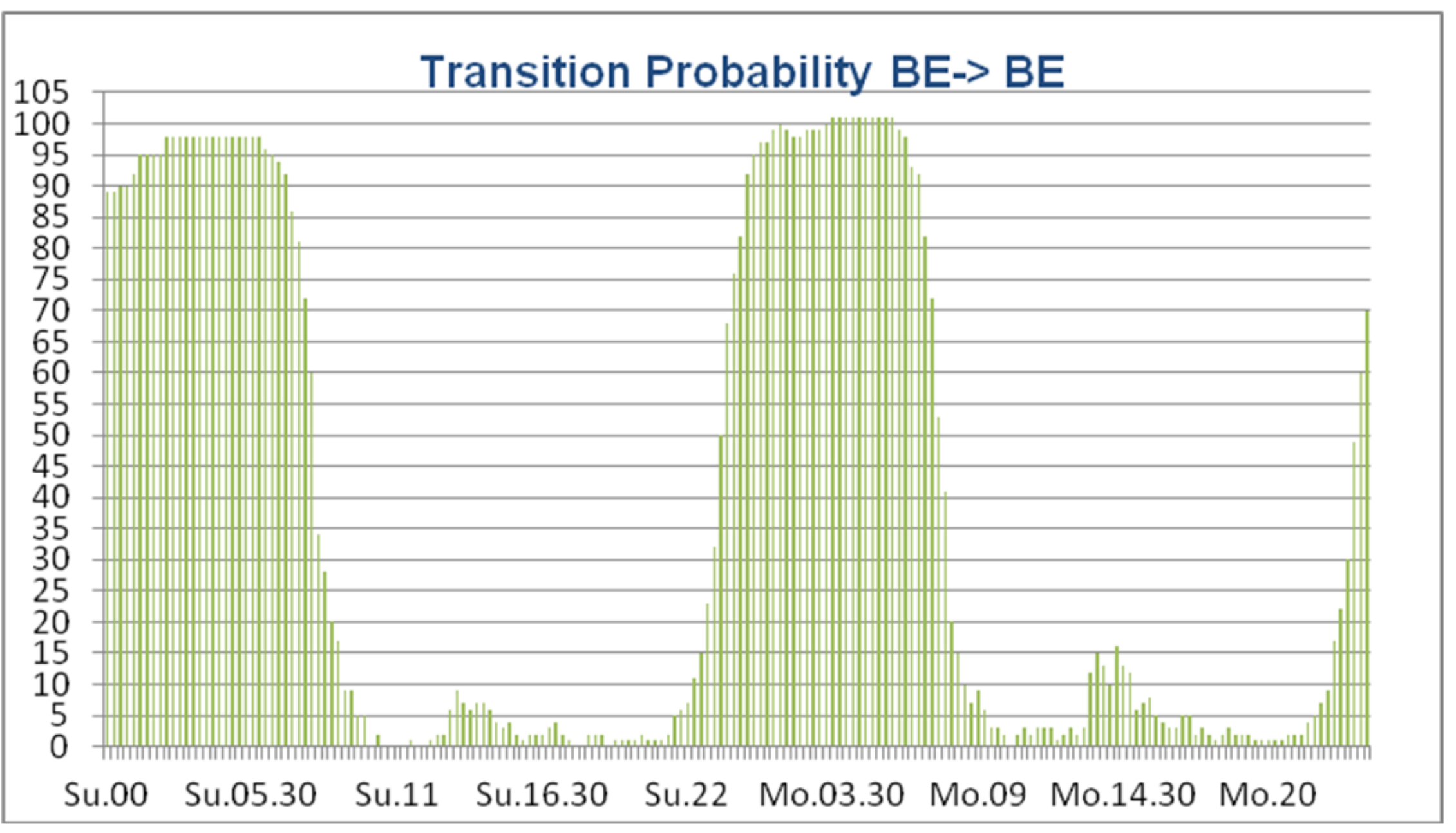




\section{MCMC}

\section{Schematic view of MCMC procedure}

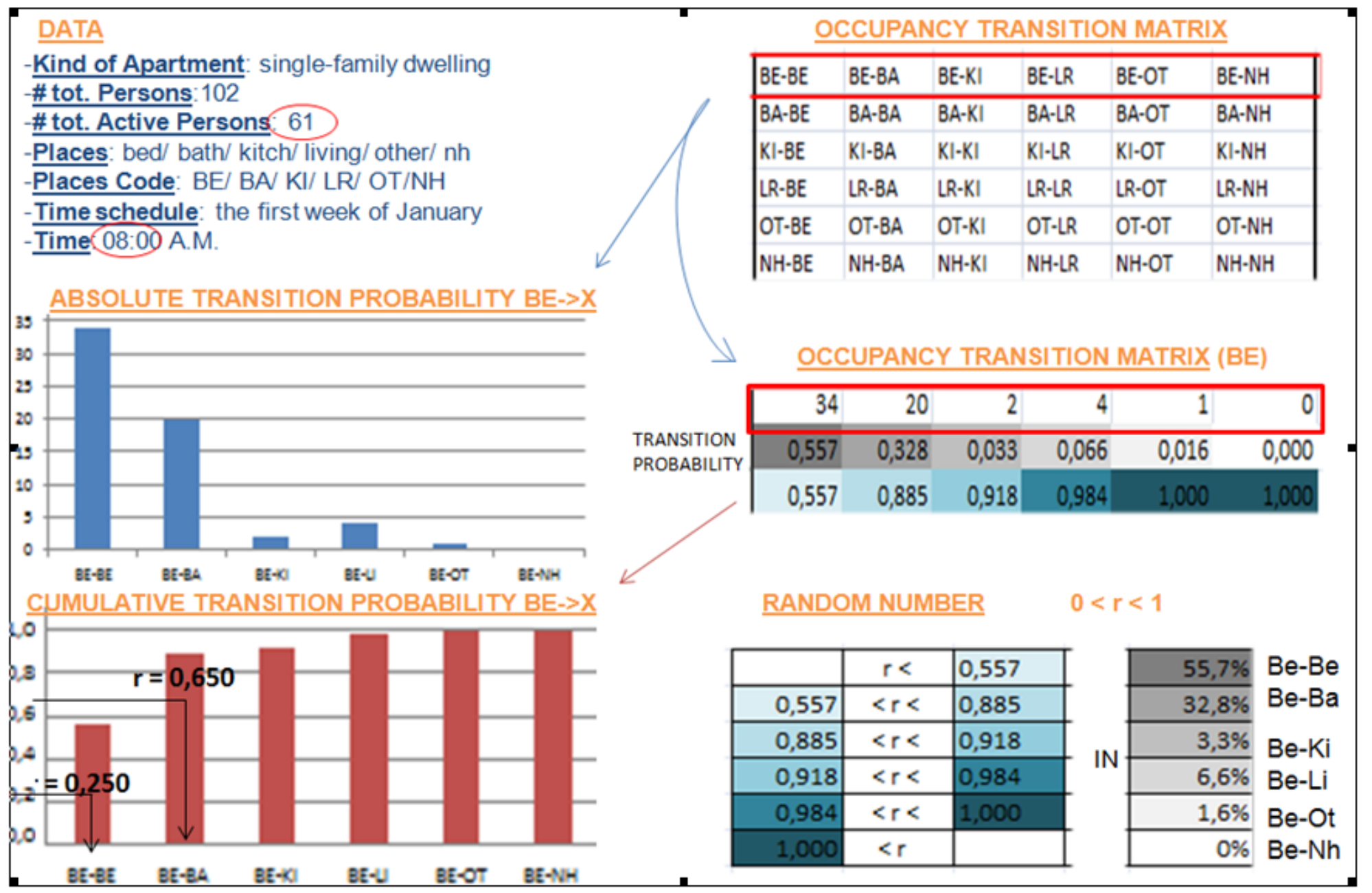




\section{MCMC results}

- Energy distribution, 2 working

- Complete year generated stochastically

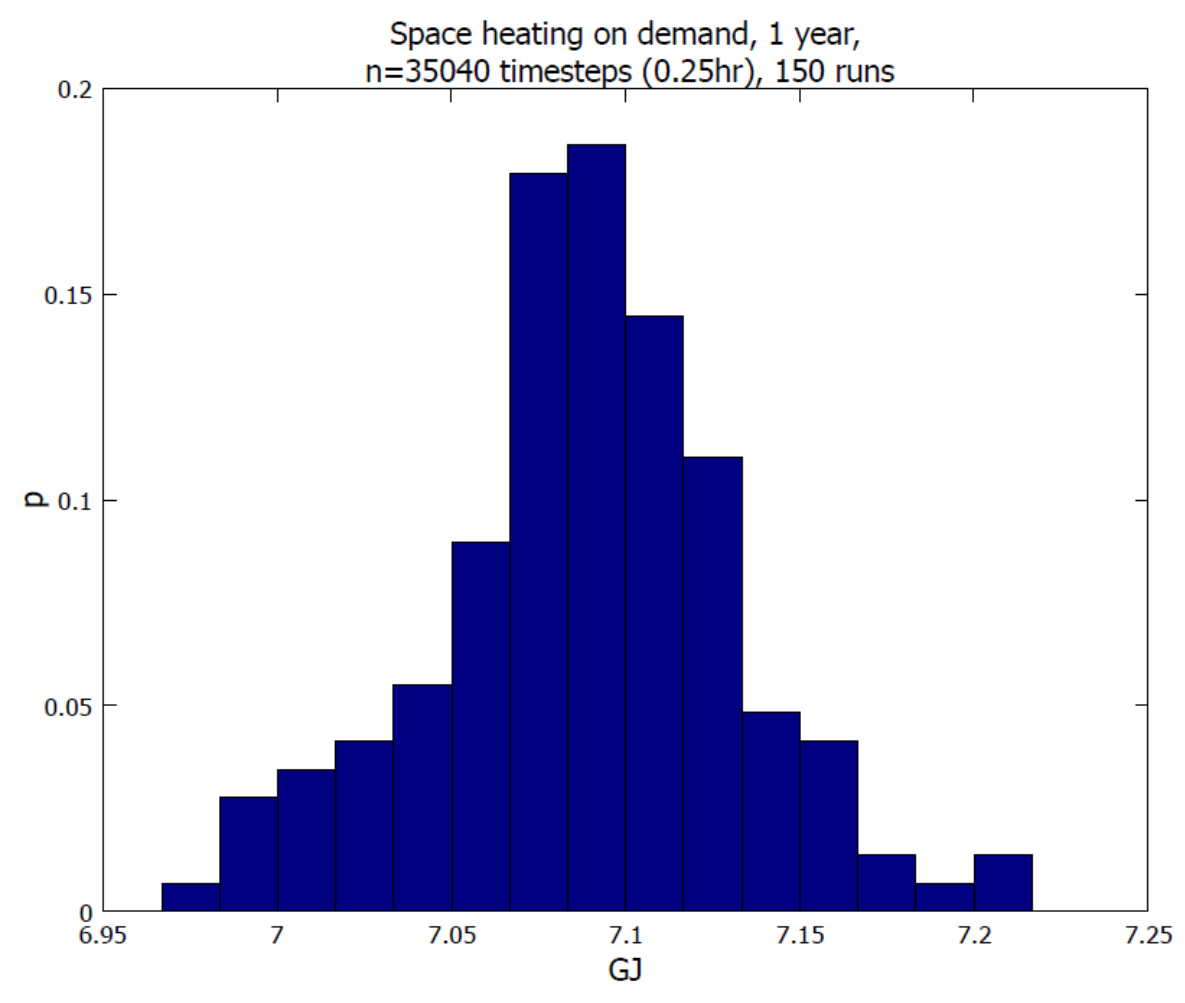

1. Yearly heating (GJ): 7.0-7.2 GJ 


\section{MCMC}

- Calculated variation in energy use is very small

- Why? Is this related to the MCMC procedure?

$\rightarrow$ Yes
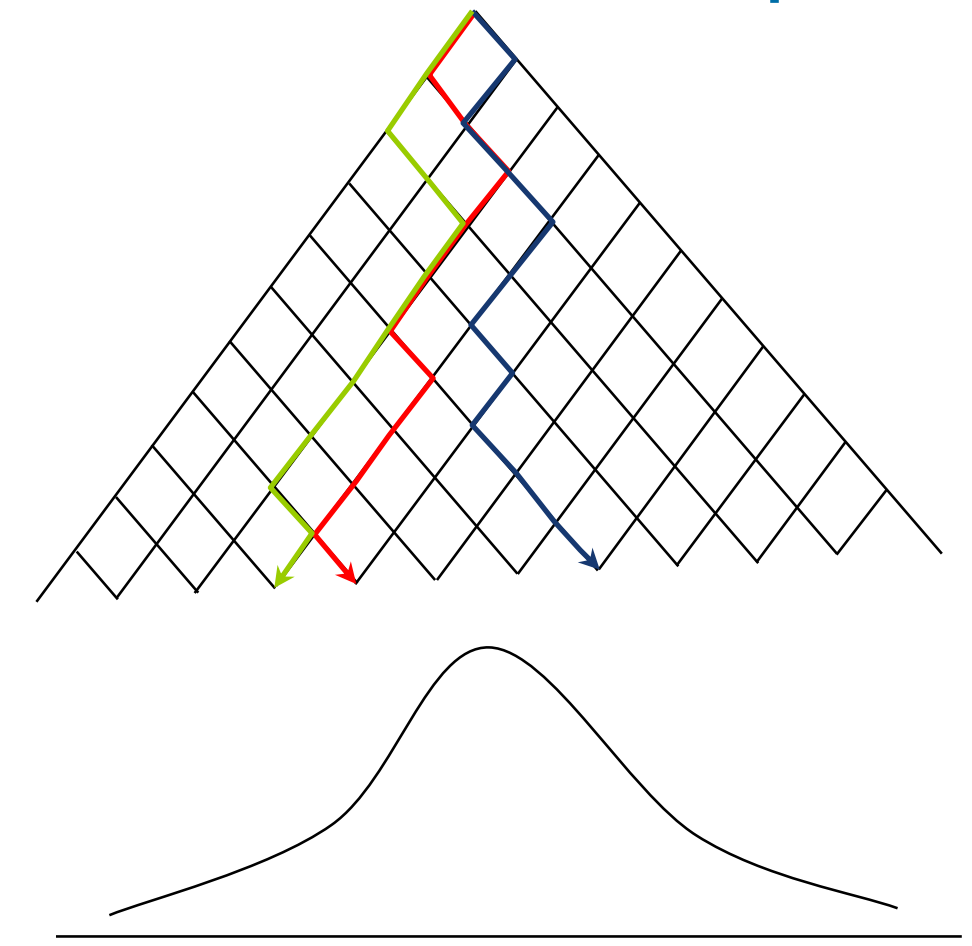

$\rightarrow$ Extreme values are unlikely to occur 


\section{MCMC}

- Extreme occupancy patterns (almost always absent/present) are not likely to be generated by MCMC for a whole year

Solution?

- $\rightarrow$ generate occupancy for one week only and use this $52 x$ for a whole year? 


\section{MCMC results}

One week generated stochastically, used 52X 2 working

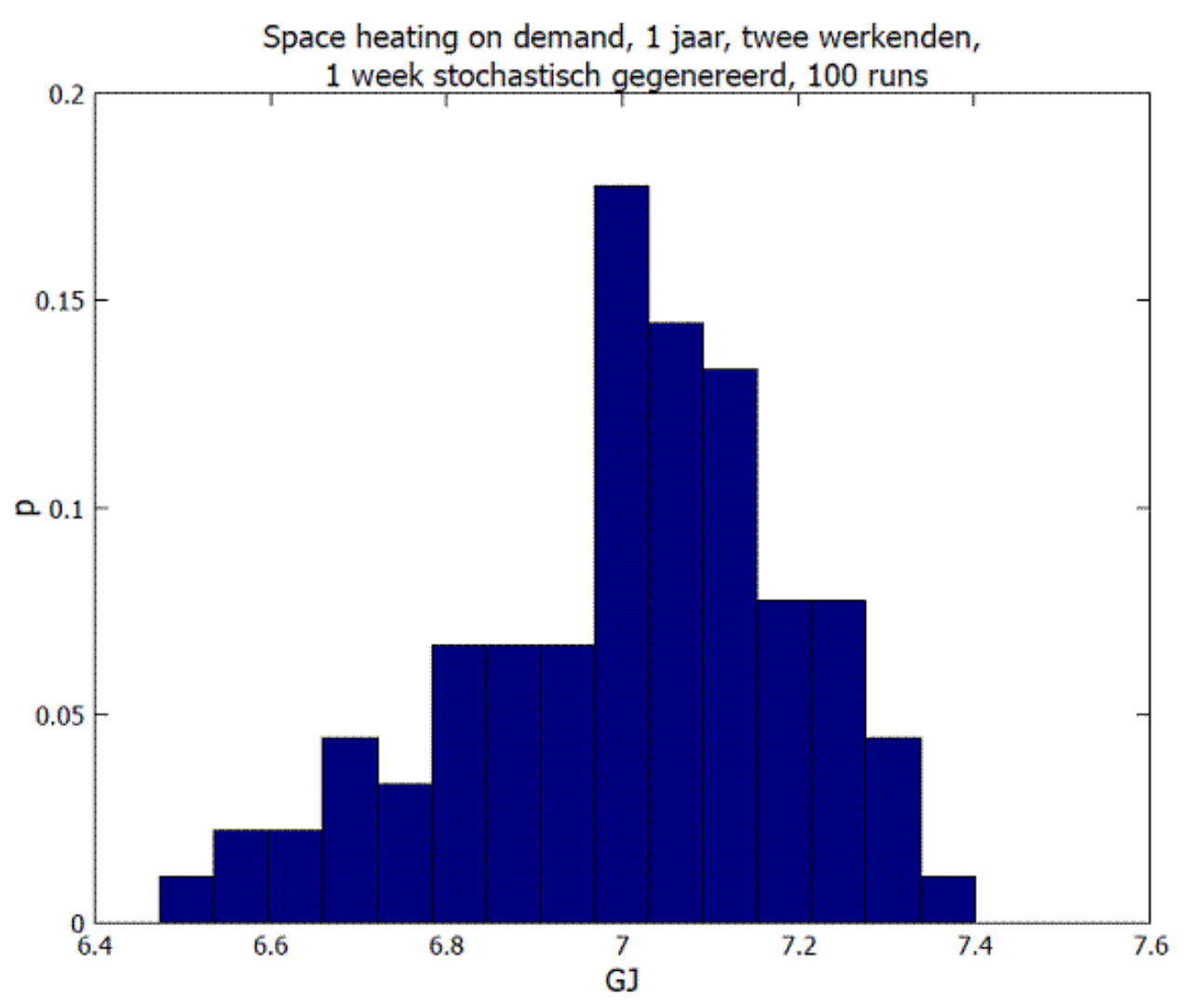

1. Yearly heating: 7.0-7.2 GJ

2. Yearly heating: $6.5-7.4 \mathrm{GJ}$ 
- The calculated variation in energy use has increased but is still not large

- Solution: $\rightarrow$ No MCMC?

Transition probabilities in the Markov-Chain are based on occupancy patterns of 100-150 different people. $\rightarrow$ variation in occupancy will be averaged out in MCMC

Monte Carlo run for deterministic profiles?

- Randomly combine deterministic occupancy profiles.

- Do this several times

- Analyze variation in energy use 


\section{Approach}

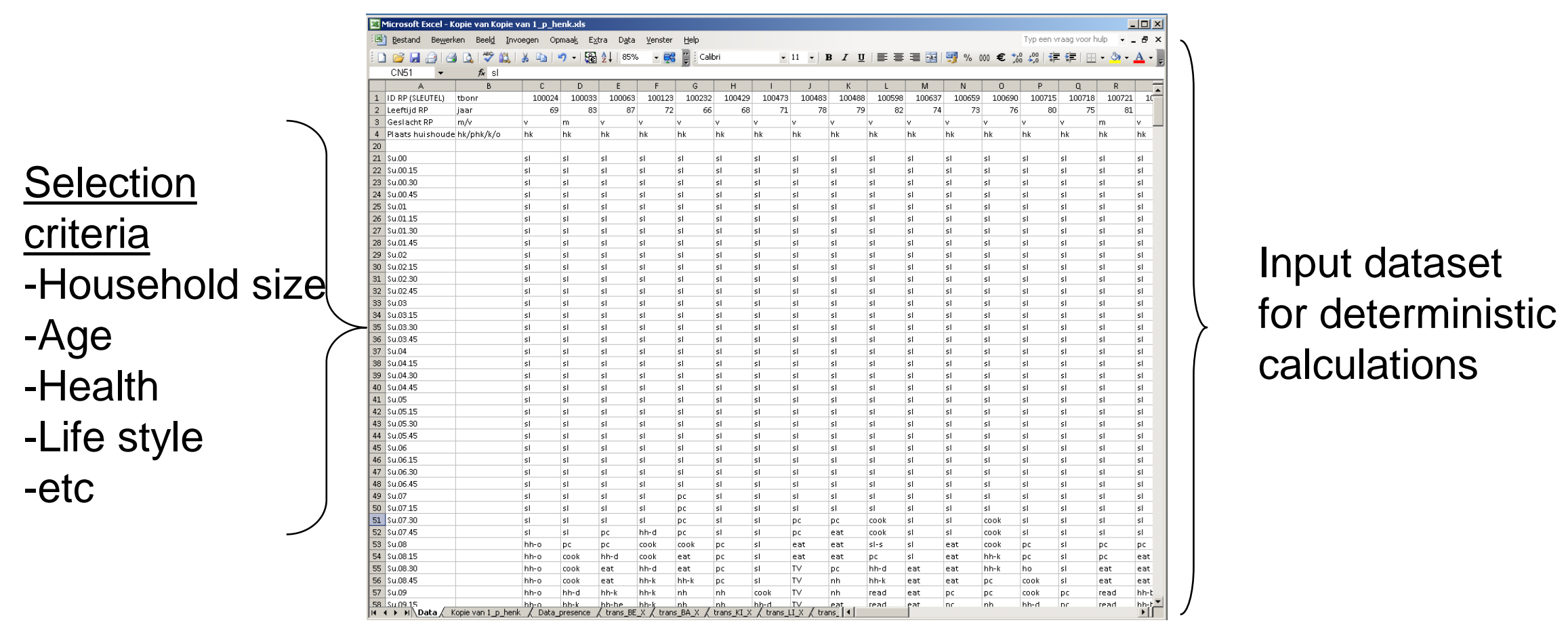




\section{Monte Carlo run for profiles}

- Deterministic profiles have been sampled repeatedly

- 2 working

1. Yearly heating: 7.0-7.2 GJ

2. Yearly heating: $6.5-7.4 \mathrm{GJ}$

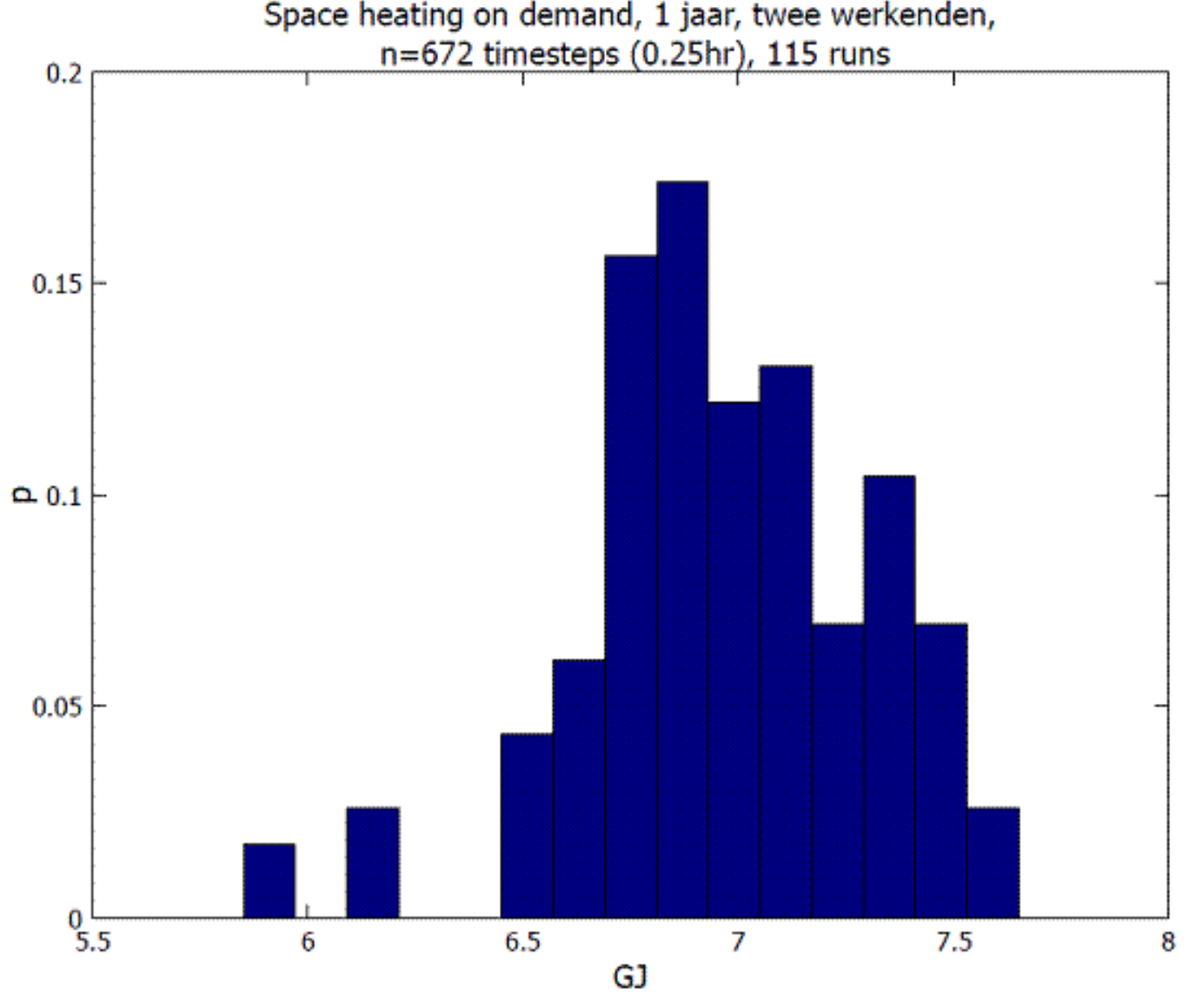

3. Yearly heating: $5.7-7.6 \mathrm{GJ}$

Space heating on demand, 1 jaar, twee werkenden, $\mathrm{n}=672$ timesteps $(0.25 \mathrm{hr}), 115$ runs 


\section{Monte Carlo run for profiles}

- The calculated variation in energy use has increased

- These values make more sense

- Apply this procedure also to "2 retired persons" and "families" 


\section{Monte Carlo run for profiles}

- Deterministic profiles have been sampled repeatedly

- 2 aged people

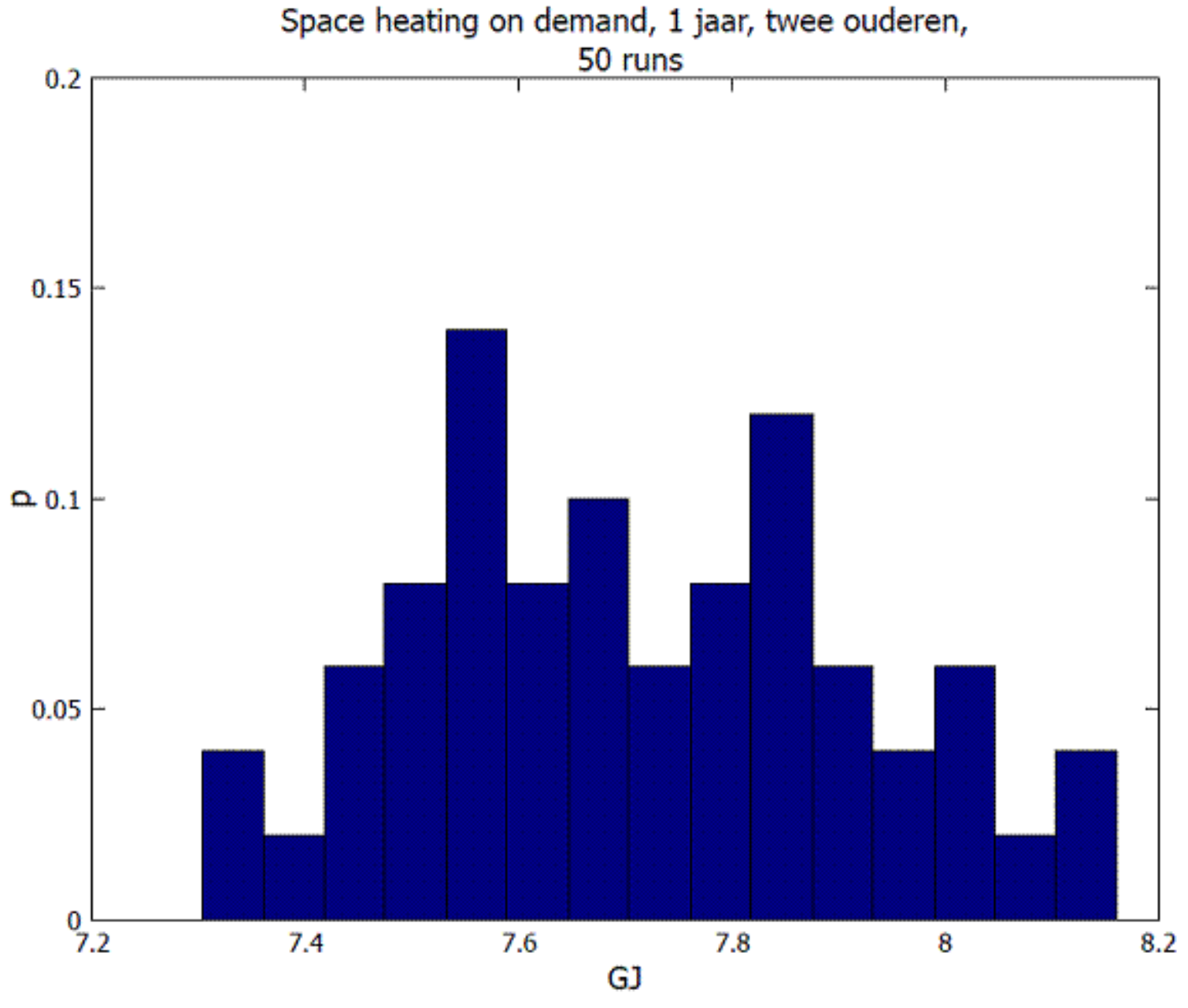




\section{Monte Carlo run for profiles}

- Deterministic profiles have been sampled repeatedly

- Families (4p.)

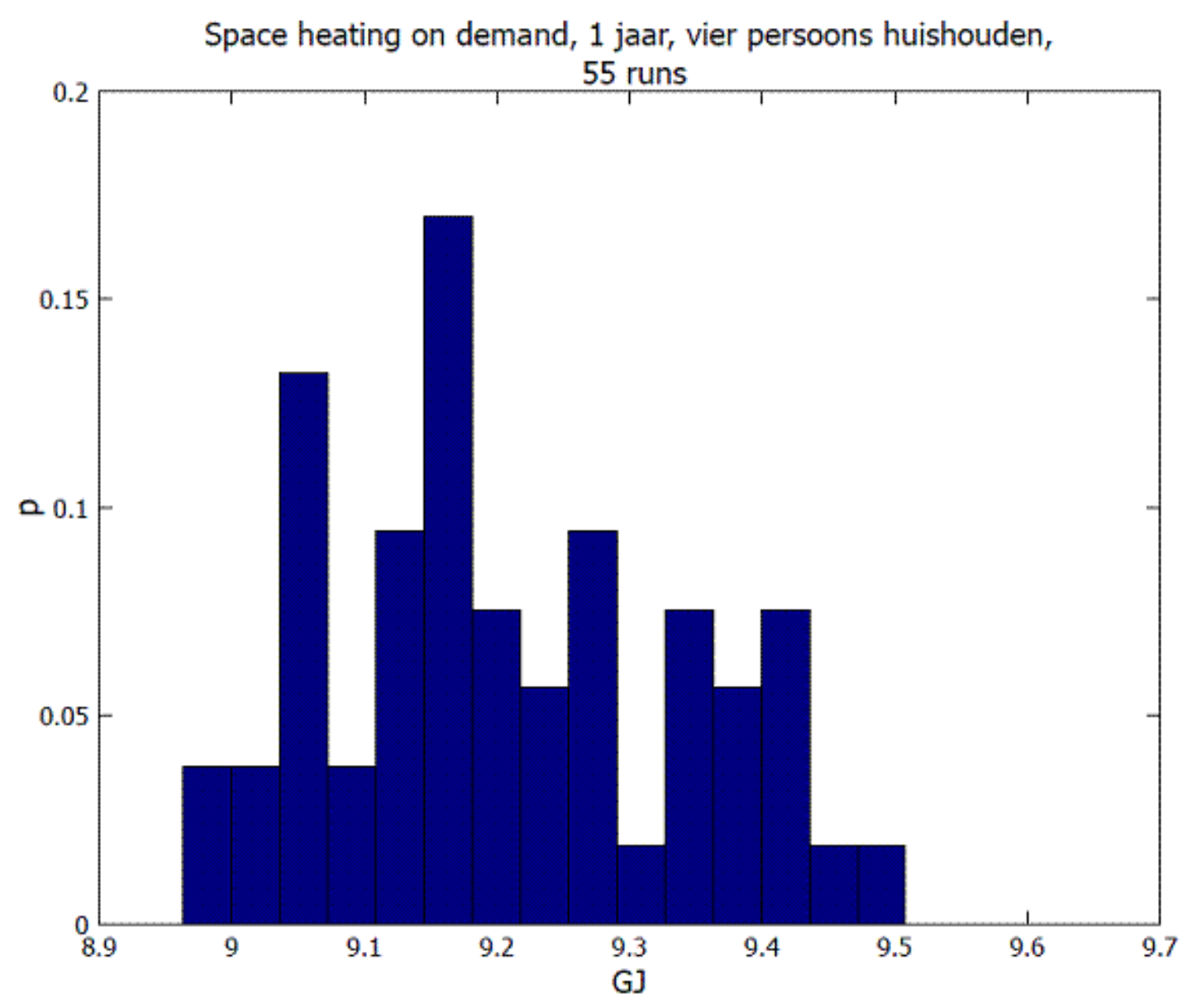




\section{Results}

- Variation in heating energy

\begin{tabular}{|l|l|l|}
\hline dwelling & occupancy & Yearly heating (GJ) \\
\hline Single-family & 2 working & $5.8-7.7$ \\
\hline Single-family & 2 retired & $7.4-8.2$ \\
\hline Single-family & 4p. family & $9.0-9.5$ \\
\hline
\end{tabular}




\section{MCMC}

\section{Schematic view of MCMC procedure}

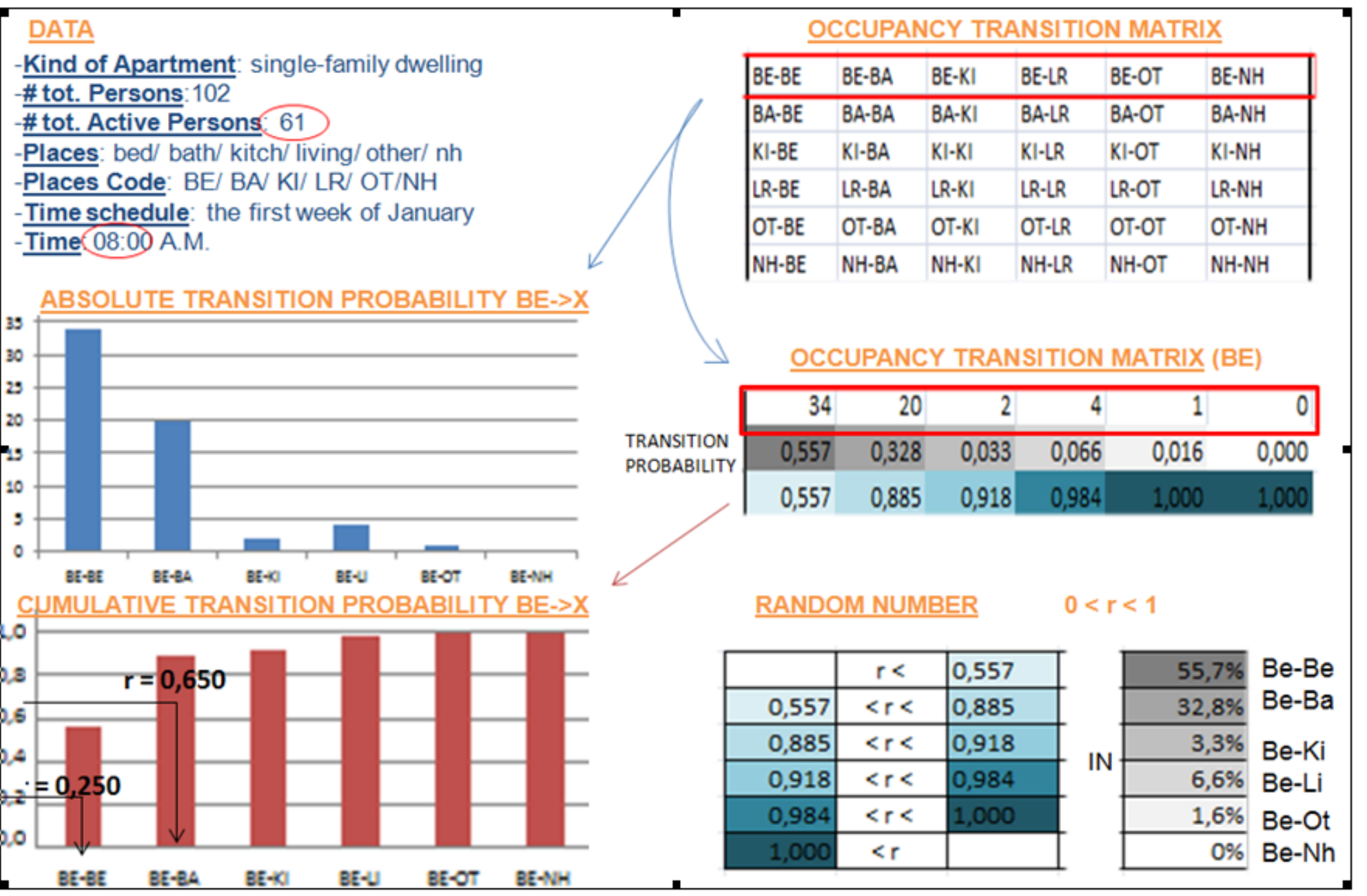




\section{Next steps}

- Coupling occupancy/building model with physiological model and psychological model

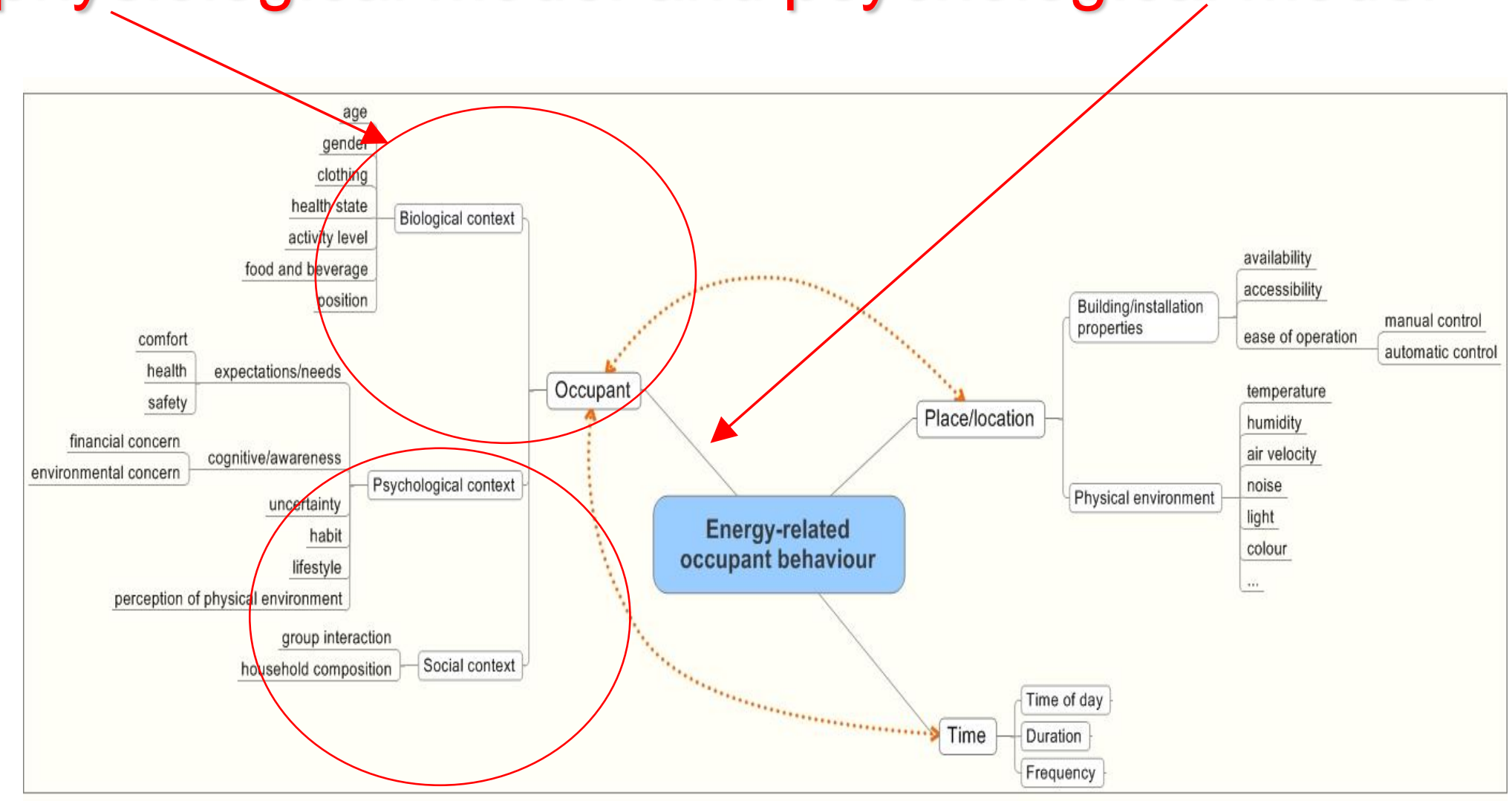




\section{Coupling with physiological model}

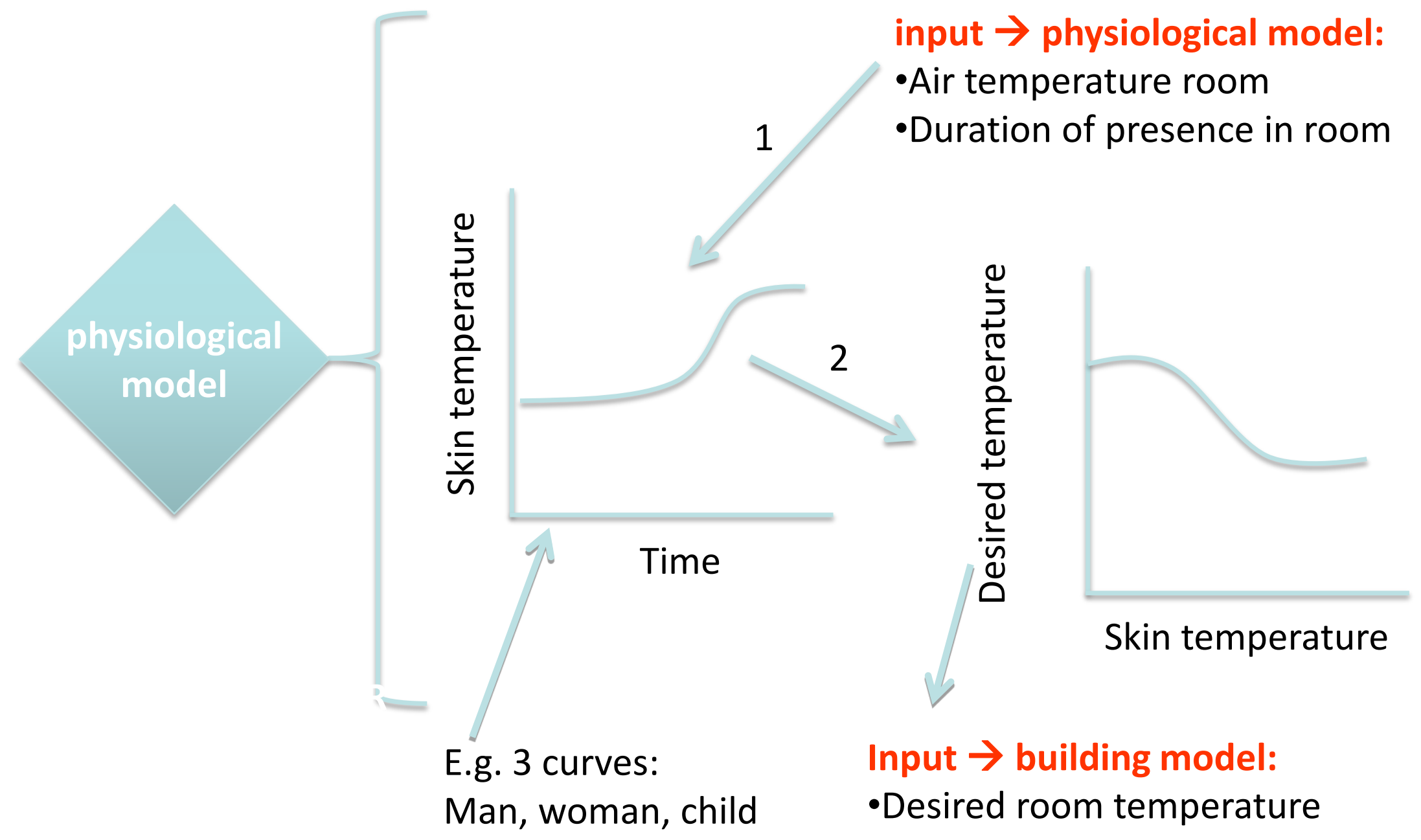




\section{Cone of uncertainty}
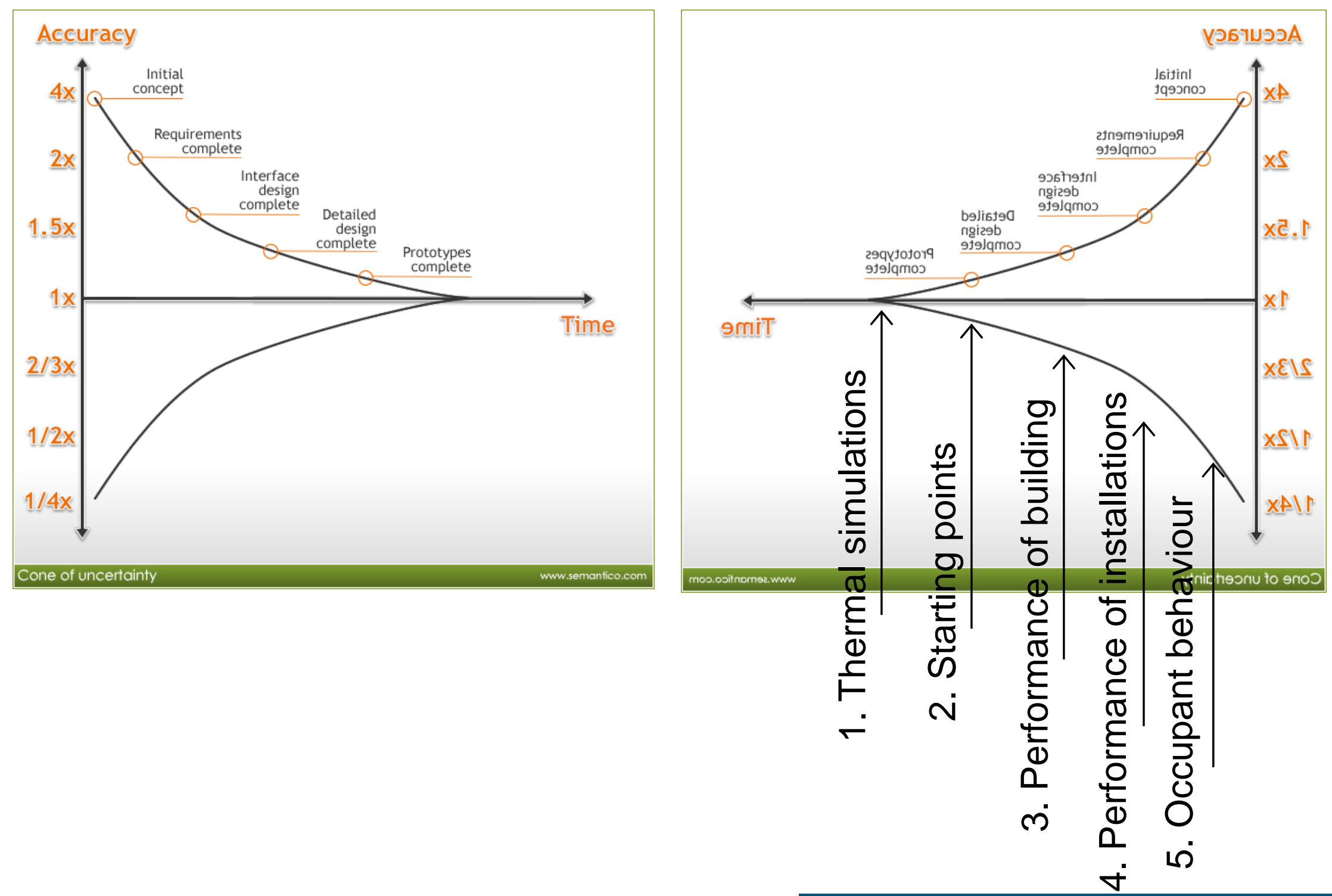


\section{Prediction of the total energy use}

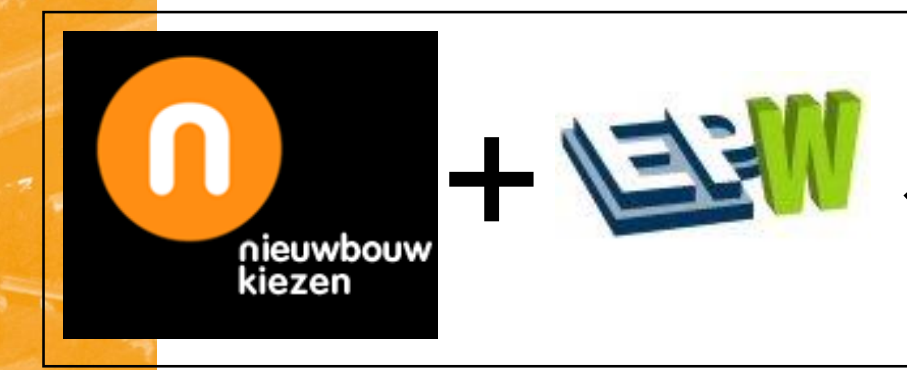

\section{Building and installation}

characteristics
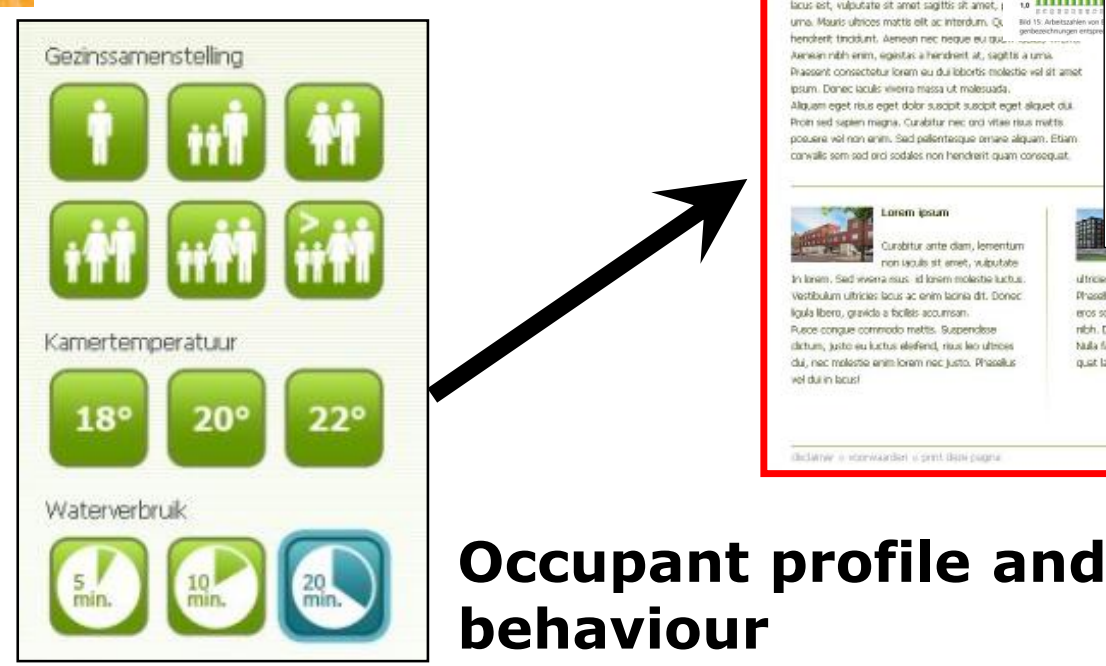

Prediction of real total energy and distribution behaviour
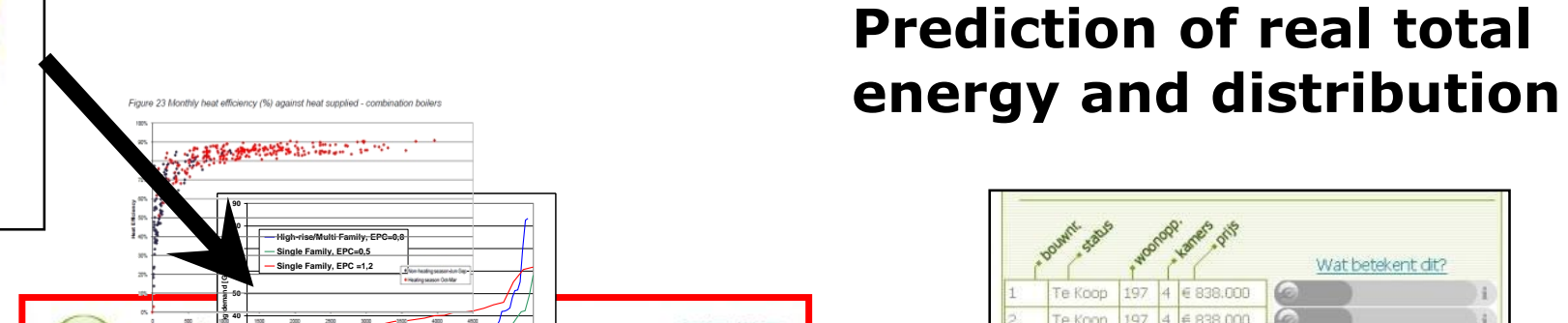


\section{From prototype to reality}

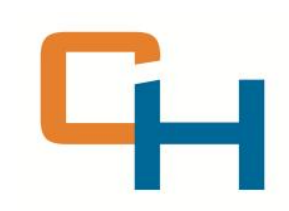

yen
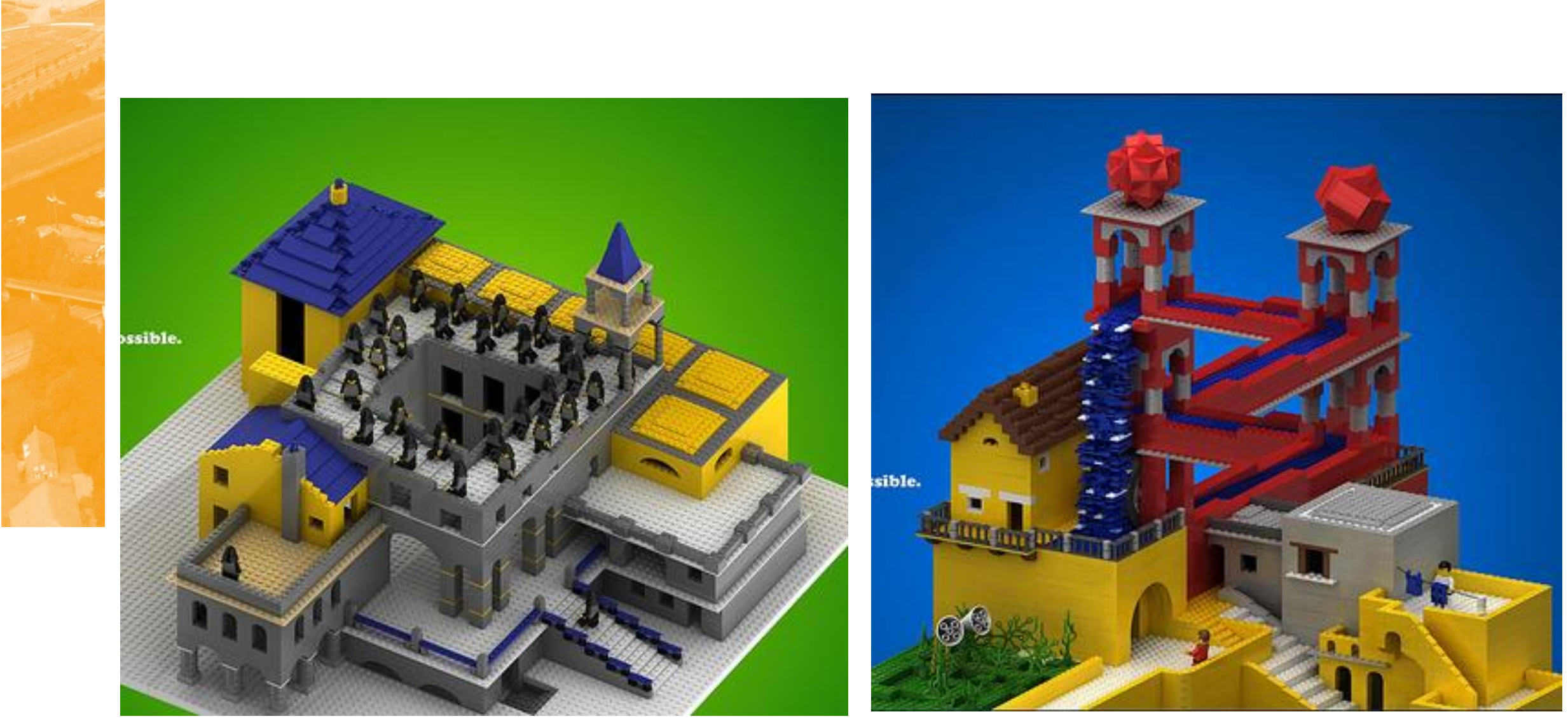